\title{
Structural Forms of the Human Amylase Locus and Their Relationships to SNPs, Haplotypes, and Obesity
}

\section{Citation}

Usher, Christina Leigh. 2015. Structural Forms of the Human Amylase Locus and Their Relationships to SNPs, Haplotypes, and Obesity. Doctoral dissertation, Harvard University, Graduate School of Arts \& Sciences.

\section{Permanent link}

http://nrs.harvard.edu/urn-3:HUL.InstRepos:17467224

\section{Terms of Use}

This article was downloaded from Harvard University's DASH repository, and is made available under the terms and conditions applicable to Other Posted Material, as set forth at http:// nrs.harvard.edu/urn-3:HUL.InstRepos:dash.current.terms-of-use\#LAA

\section{Share Your Story}

The Harvard community has made this article openly available. Please share how this access benefits you. Submit a story. 


\title{
Structural forms of the human amylase locus
}

\section{and their relationships to SNPs, haplotypes, and obesity}

A dissertation presented

by

\section{Christina Leigh Usher}

\author{
to

\section{The Division of Medical Sciences} \\ in partial fulfillment of the requirements \\ for the degree of \\ Doctor of Philosophy \\ in the subject of \\ Genetics and Genomics
}

Harvard University

Cambridge, Massachusetts

March 2015 


\section{(C) 2015 Christina Leigh Usher}

All rights reserved. 


\title{
Structural forms of the human amylase locus and their relationships to SNPs, haplotypes, and obesity
}

\begin{abstract}
Hundreds of human genes reside in structurally complex loci that elude molecular analysis and assessment in genome-wide association studies (GWAS). One such locus contains the three different amylase genes ( $A M Y 2 B, A M Y 2 A$, and $A M Y 1)$ responsible for digesting starch into sugar. The copy number of $A M Y 1$ is reported to be the genome's largest influence on obesity, yet has gone undetected in GWAS. Using droplet digital PCR (ddPCR), sequence analysis, and optical mapping, we characterized eight common structural forms of the amylase locus, their mutational histories, and their relationships to SNPs. We found that $A M Y 1$ copy number has a unique distribution undetectable to earlier methods that can be understood from an underlying set of structural forms and their allele frequencies. Despite a history of recurrent structural mutations, $A M Y 1$ copy number has maintained partial correlations to nearby SNPs; these SNPs do not associate with body mass index (BMI). To directly test for association, we measured amylase gene copy number using ddPCR in 1,000 Estonians selected for being either obese or lean and in two cohorts totaling $\sim 3,500$ individuals using sequence analysis. We had $99 \%$ power to detect even the lower bound of the reported effects on BMI and obesity, yet found no association. This study model of using multiple methods to analyze the copy number, structural haplotypes, and surrounding SNP haplotypes of multi-allelic variants will likely facilitate more robust disease association results in future studies.
\end{abstract}




\section{Acknowledgements}

My graduate school experience has been a long journey that would have been even longer without the help of my mentors, friends, and family. I would first like to thank Steve McCarroll for being my mentor. It has been an honor being his first graduate student, and quite possibly, his most stubborn. Yet, despite my greatest protestations, he never stopped giving me good advice that I would secretly follow and good ideas that drove my projects forward. His best gift has been his whole-hearted approval of my career aspirations and the connections to get me there.

I would like to thank the members of the McCarroll lab for being fun, supportive, and knowledgeable. I would like to thank the first generation members Cindy Liu, Tom Mullen, and Elizabeth Fels for being a friend, a motivator, and a ridiculously good assistant, respectively. I'd like to thank the newer folks Curtis, Vanessa, Heather, Evan, Dominique, Arpiar, and Michelle for keeping the good vibes going. Of course, I have to give shout outs to Bob Handsaker and Jim Nemesh for being our omnipotent computational specialists. And last, but not least, I have to thank my officemates Linda Boettger, Aswin Sekar, Nolan Kamitaki, and Avery Davis for being more friends than coworkers. Know that even though I grumpily wore my headphones every time you guys were talking, I was still listening. 
I am grateful for having had the opportunity to work with and learn from a wonderful group of collaborators: the labs of Joel Hirschhorn, Timothy Frayling, Charles Lee, and BioNano. It was also a privilege to be guided by my Dissertation Advisory Committee Jesse Gray, Mark Daly, Alkes Price, and James Gusella. I would also like to thank Mark Daly, Terence Capellini, Matt Warman, and John Armour for kindly agreeing to be a part of my dissertation examination committee. And lastly, I'd like to thank the members of my undergraduate lab David Bloom, Nicole Giordani, Dacia Kwiatkowski, Cameron Lilly, and Levi Watson for making science look so fun that I wanted to pursue it in graduate school.

I am also indebted to my family (Susan, Michael, Kevin, David, and Joyce Usher, and Heinz and Helga Meincke) for keeping me on track by asking me when I'm going to graduate even more times than the BBS administrative office. But honestly, my life wouldn't have been as successful without them shipping up to Boston for condo repairs and keeping up long-distance support through cell phones and Facebook. 


\section{Table of Contents}

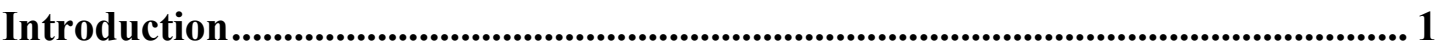

Multi-allelic CNVs....................................................................................................... 2

The rationale of the dissertation .................................................................................. 8

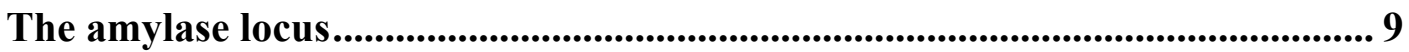

Chapter 1. Measuring the amylase locus ....................................................................... 21

Optimizing the measurement of $A M Y 1$................................................................. 23

The distribution of amylase gene copy number ....................................................... 29

The structural alleles of the amylase locus................................................................. 31

Chapter 2. Assembling the alleles of the amylase locus............................................. 35

Published assemblies ......................................................................................................... 35

Novel assemblies ............................................................................................................. 39

Chapter 3. SNP correlations to the amylase locus ...................................................... 45

SNPs capturing structural haplotypes ....................................................................... 47

SNPs capturing copy number ...................................................................................... 48

Chapter 4. Associating the amylase locus to obesity................................................... 49

Discussion ........................................................................................................................ 54

Discussion of the previous report.......................................................................... 54

Discussion of my proposed study model...................................................................... 57

Future Directions............................................................................................................... 61

Appendix A. Dissecting the timing \& origin of gene expression variation ........... 64

Appendix B. Experimental Methods .......................................................................... 87 


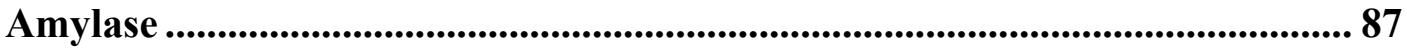

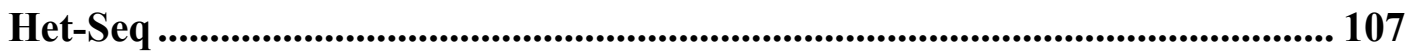

Appendix C. Protocols for amylase genotyping using ddPCR ............................ 117

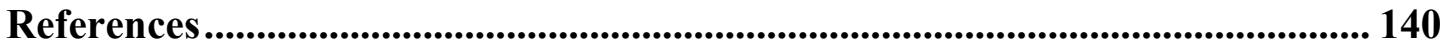




\section{Introduction}

Human genomes have thousands of deletion and duplication polymorphisms. These so-called copy number variants (CNVs) cause as many as $\sim 0.78 \%$ of base pairs to differ in copy number between any two individuals' genomes ${ }^{1}$. The first indication that these copy number alterations could influence human phenotypes came from sporadic diseases, now termed genomic disorders, caused by de novo structural alterations ${ }^{2}$. The dominant mutations responsible tended to alter gene dosage and occur in regions of genomic instability caused by segmental duplications and low copy repeats ${ }^{3}$. It was not long until some inherited genomic disorders and mendelian diseases were linked to inherited CNVs in linkage studies ${ }^{4-6}$. However, despite the evidence of inheritance, early studies of $\mathrm{CNV}$ in normal individuals still referred to common CNVs as recurring, as if these CNVs had independent origins ${ }^{7}$ and were affecting as yet undefined phenotypes.

Though rare and de novo CNVs have well-known roles in disease, with many

having strong odds ratios of $2-30^{8-10}$, we now know from microarray-based genome-wide CNV studies in phenotypically normal populations that most of the CNV in any individual's genome arises from a reservoir of polymorphisms that are common ${ }^{11}$, stably 
inherited $^{11}$, and mostly benign ${ }^{12}$. Most deletions and simple duplications are now routinely assessed in genome-wide association studies, either directly with arrays ${ }^{13}$ or with imputation ${ }^{14,15}$. However, there is a class of CNV that has been left behind by these genomic advances.

\section{Multi-allelic CNVs}

Out of the $\sim 8500 \mathrm{CNV}$ s found in Phase 1 of the 1000 Genomes Project ${ }^{16}, 1,356$ were more complex than simple deletions and duplications. These multi-allelic CNVs (mCNVs) varied widely in copy number, in patterns that imply the existence of three, four, five, or more segregating alleles. In that particular data set, 121 appeared to have four or more alleles, and 45 appeared to have five or more ${ }^{16}$.

When these multi-allelic CNVs are visualized by fiber FISH, they have often been found to involve tandem or inverted duplications of a genomic segment ${ }^{17,18}$. Some of these duplications have been estimated (from sequencing data) to have up to about 50 copies, though the great majority appear to be present in copy numbers of $0-12^{16,19}$. Many mCNVs are more complex than copy number can quantify, because they also involve additional duplications, deletions, and inversions, sometimes affecting multiple genes $^{11,19-22}$. An apt example is the $17 \mathrm{q} 21.31$ region, associated with fertility ${ }^{23}$ and neurological diseases ${ }^{24,25}$. It has nine alleles that affect five genes through various numbers of duplications, sequence changes, and a single large-scale inversion ${ }^{21,22}$. It is one of the only mCNVs to be assembled into haplotypes on a sequence-level; however, investigations into other loci, such as the amylase locus, $F C G R 3 B / 3 A, C C L 3 L 1$, and $C 4$, indicate that $\mathrm{mCNV}$ s commonly have other variants within them ${ }^{17,26-29}$ (Figure I-1). 


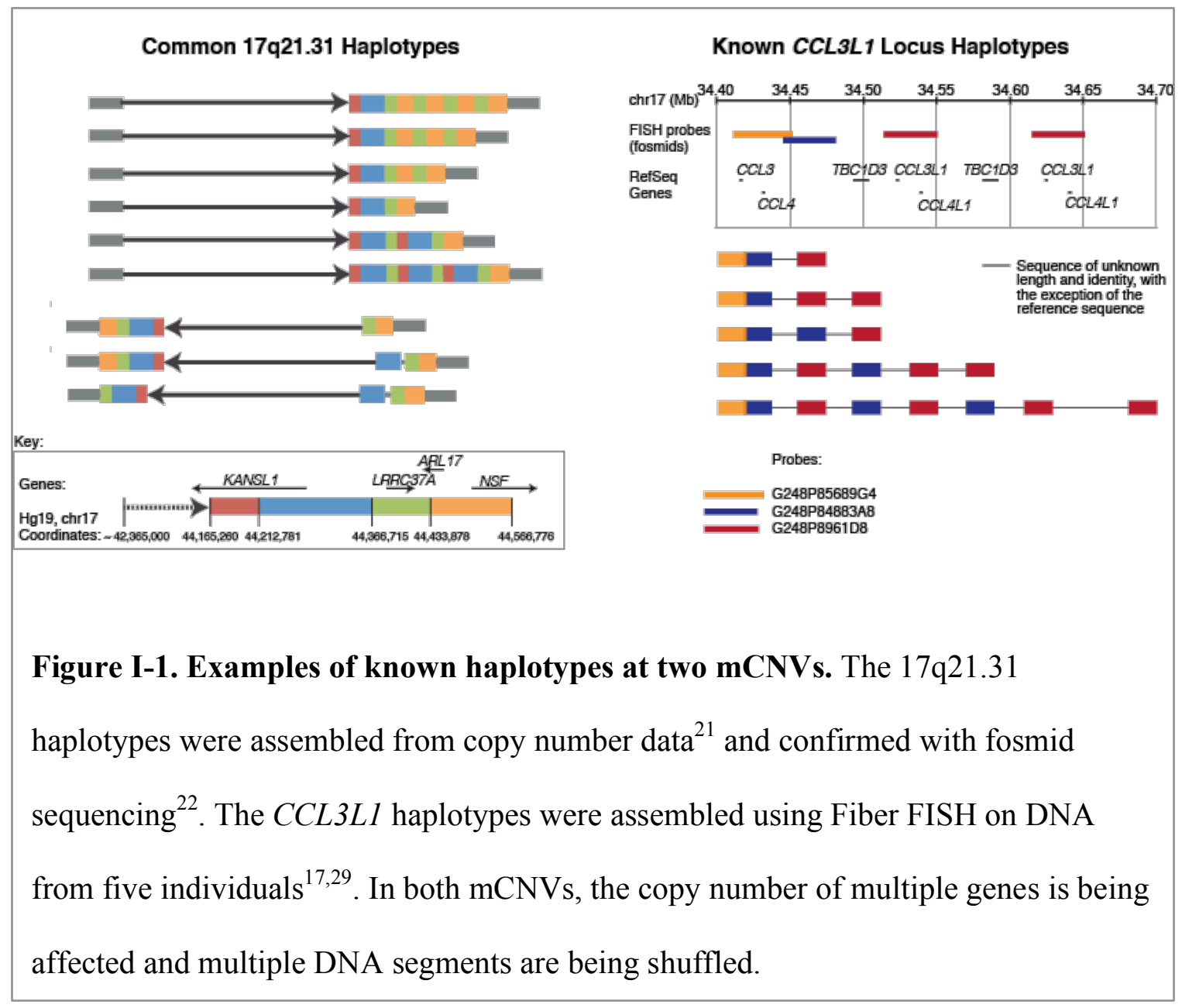

The large sequence modifications characteristic of mCNVs can disrupt the genes that overlap them, influencing disease and evolution in a variety of ways. All CNVs, simple and multi-allelic, may change the coding sequence of a gene ${ }^{30,31}$, create paralogs that can diverge to create new or specialized functions ${ }^{32}$, and alter the overall gene expression level $\mathrm{l}^{31,33,34}$. mCNVs can have an even greater impact because they can have many duplications, and these effects are multiplied when several genes and non-coding elements are in the same mCNV locus, all being affected by the same, or different, disruptions. In fact, $\mathrm{mCNVs}$ account for $88 \%$ of the gene dosage variation in humans ${ }^{16}$. 
In theory, an mCNV's impact on a disease should be easier to parse than that of a SNP. Many mCNVs have of a series of alleles with different numbers of gene duplications, creating an allelic series of growing phenotypic impact and testable hypotheses about how the mCNV is contributing to the phenotype. Their multi-allelic nature also ensures that they cannot be in high LD with any single SNP. Thus, their signal of association is easier to pinpoint in the genome, and their roles in disease are easier to decipher once an association has been found.

Yet only a handful of $\mathrm{mCNV}$ associations have been reported and most were ascertained through candidate gene studies, with only a few genes receiving most of the attention due to their known roles in immunity and disease progression: FCGR3B (binds the Fc region of gamma immunoglobulins), CCL3L1 (ligand of the co-receptor for the HIV virus), beta-defensins (cluster of microbicidal and cytotoxic peptides), $H B A 1 / 2$ ( $\alpha$ chain of hemoglobin), and $C 4$ (part of the complement pathway) ${ }^{27,35-47}$.

On top of the relatively few genes that are being investigated, many of these associations have been resistant to replication in independent cohorts with independent investigators $^{48}$, with some associations disappearing when different genotyping methods

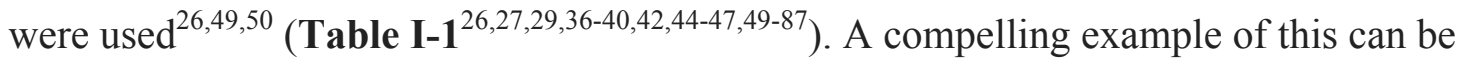
found in the Wellcome Trust Case Control study, which used an array-based genotyping technology to perform a genome-wide association study on eight common diseases. Despite good copy number measurements (that clustered at integers) and a large sample size (2000 cases), it did not replicate three previously published associations (FCGR3B on rheumatoid arthritis, CCL $3 L 1$ on rheumatoid arthritis, B-defensins on Crohn's $\left.\operatorname{disease}^{39,46,47}\right)$. 
Table I-1. Reported associations with attempted replications. Nearly every

reported disease association has failed to replicate in at least one follow up study.

Small sample sizes may be behind some of these non-replications, but not all. Entries

are referenced by the first author and in the above text.

\begin{tabular}{|c|c|c|c|c|}
\hline Gene & Disease & $\begin{array}{l}\text { First study to find an } \\
\text { effect by measuring } \\
\text { copy number }\end{array}$ & Replication - independent cohort & $\begin{array}{l}\text { Replication - precise } \\
\text { genotyping method }\end{array}$ \\
\hline \multirow[t]{4}{*}{$F C G 3 R B$} & $\begin{array}{l}\text { systemic lupus } \\
\text { erythematous }\end{array}$ & Fanciulli (536) & $\begin{array}{c}\text { Molokhia (134), Mamtani (149), Willcocks } \\
\text { (159), Willcocks (171), Aitman (187 sib } \\
\text { pairs), Lv (202), Chen (846) }\end{array}$ & $\begin{array}{c}\text { Neiderer (210), Neiderer } \\
\text { (240), Morris (365 trios), } \\
\text { Neiderer (880) }\end{array}$ \\
\hline & Sjögren's syndrome & Mamtani (61) & Halderson (124), Lester (174) & \\
\hline & $\begin{array}{l}\text { anti-neutrophil } \\
\text { cytoplasmic-antibody- } \\
\text { associated vasculitis }\end{array}$ & Fanciulli (77) & Willcocks (556) & Niederer (567) \\
\hline & Rheumatoid arthritis & McKinney (768) & $\begin{array}{c}\text { Breunis (112), Mamtani (158), Graf (197), } \\
\text { McKinney (250), Marques (518),McKinney } \\
\text { (643), Chen (948) }\end{array}$ & $\begin{array}{c}\text { Robinson (1188), } \\
\text { Wellcome Trust Case } \\
\text { Control Consortium } \\
(2000)^{*}\end{array}$ \\
\hline FCGR3A & Rheumatoid arthritis & Chen (948) & Thabet (945) & Robinson (1188) \\
\hline \multirow[t]{3}{*}{$C C L 3 L 1$} & HIV phenotypes & Gonzalez (1132) & $\begin{array}{l}\text { Bhattacharya (46), Meddows-Taylor } \\
\text { (46),Lee (48), Nakajima (95), Larson (153), } \\
\text { Dolan (165), Shostakovich-Koretskaya } \\
\text { (178), Shao (184), Rathore (196), Huik } \\
\text { (208), Dolan (240), Kuhn (460), Gonzalez } \\
\text { (470), Urban (1855) }\end{array}$ & Aklillu (1134) \\
\hline & Kawasaki Disease & Burns (164) & Mamtani (133), Kim (459) & - \\
\hline & Rheumatoid arthritis & McKinney (1136) & - & $\begin{array}{c}\text { Carpenter (274), Nordang } \\
\text { (900), Wellcome Trust } \\
\text { Case Control Consortium } \\
\text { (2000) }\end{array}$ \\
\hline \multirow[t]{2}{*}{ B-defensins } & Crohn's disease & Fellermann (85) & Fellermann (165), Bentley (466) & $\begin{array}{c}\text { Aldhous (1500), } \\
\text { Wellcome Trust Case } \\
\text { Control Consortium } \\
(2000)^{*}\end{array}$ \\
\hline & Psoriasis & Hollox (179) & - & $\begin{array}{l}\text { Hollox (319), Stuart } \\
\text { (1396), Stuart (2616) }\end{array}$ \\
\hline \multirow[t]{3}{*}{ C4 } & $\begin{array}{l}\text { systemic lupus } \\
\text { erythematous }\end{array}$ & Yang (233) & Lv (924) & $\begin{array}{c}\text { Boteva (501), Boteva } \\
\text { (527) }\end{array}$ \\
\hline & Type 1 diabetes & Mason (50) & Mason (110), Pani (220 families) & - \\
\hline & Cardiovascular health & Arason (233) & Blasko (142) & - \\
\hline A-globin & Severe malaria & Allen (249) & - & $\begin{array}{c}\text { Lell (100), } \\
\text { Mockenhaupt(301), } \\
\text { Williams (655), May } \\
\text { (2591) }\end{array}$ \\
\hline
\end{tabular}

Red $=$ no association found $(\mathrm{p}<0.05$, unless otherwise stated $)$

Orange $=$ association found in the opposite direction

Gray $=$ studies that began the work of investigating surrounding LD

$(\#)=$ number of cases included in the study, provided to give an estimate of the power each cohort had. Controls normally equaled the number of cases

*Non-significant after an allowance for multiple testing

$\wedge^{\wedge}$ Studies with shared cohorts were excluded from this group 
Another example is $F C G R 3 B$ 's impact on anti-neutrophil cytoplasmic antibody (ANCA)-associated vasculitis, where three studies found three different results: association to a lower copy number, association to a higher copy number, and no association at all - despite the studies sharing the same cohort ${ }^{26,37,55}$ (in addition to other samples). These controversies likely have many causes, some global and some locusspecific. However, they most likely stem from the difficulties of measuring the copy numbers of $\mathrm{mCNVs}^{48}$ (referred to as "genotyping") and from not accounting for locus structure.

\section{Imprecise genotypes}

While the genotyping of simple CNVs and SNPs is mature - they are routinely analyzed by arrays and imputation ${ }^{15,88}$ - genotyping multi-allelic CNVs requires more sensitivity. An assay measuring the copy number of an mCNV must be able to discriminate within its range of copy numbers. The difference between a copy number of 4 and one of 5 is only $20 \%$, a difference that can be difficult to detect using techniques like real-time PCR, a method that is used for the majority of $\mathrm{mCNV}$ association studies ${ }^{48}$.

The rough estimates of copy number that are produced with qPCR can result in a bell-shaped distribution of genotypes, characterized by no clustering at integers ${ }^{48-50,89,90}$. This distribution can hide technical confounds caused by genotyping-batch effects, DNAisolation batch effects, and other, unknown factors - some or all of which are suspected to have caused false associations at particular loci ${ }^{49,50,89,90}$ (Figure I-2). In addition, by adding noise to an assay, the rough estimates of copy number can further hide the signal of association, requiring larger patient cohorts and more genotyping replications to find the hidden signal. Imprecise genotypes can be avoided by using an appropriate 
technology, such as the paralog ratio test (PRT), droplet digital PCR (ddPCR), or sequence analysis (with programs like Genome STRiP). However, these techniques still require knowledge of the genetic structure of the locus.
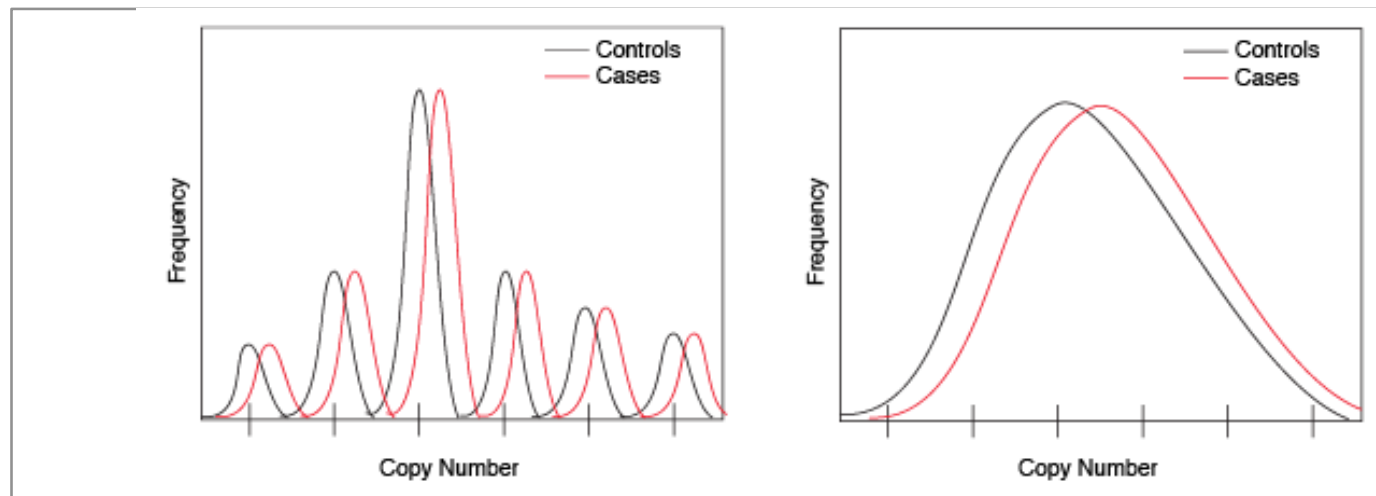

Figure I-2. Batch effects can lurk in imprecise copy numbers. To the left, a small technical artifact causing the cases' copy numbers to be shifted right can be identified (and accounted for), because the copy numbers of the cases are clustering at integers +0.2 , which likely does not reflect real biology. In the imprecise copy numbers to the right, the small technical artifact can appear to be a real effect.

\section{Locus structure}

Many mCNVs are not only simple tandem duplications, but are complex, in that they also involve additional duplications, deletions, and inversions ${ }^{11,19-22}$. The presence and number of these features vary by allele, and designing a copy number assay by only using the reference genome can result in inaccurate genotypes if an assay is in, or crosses a breakpoint of, one of these other variants. Other locus-specific features, such as 
extensive homology in the resident genes, can interfere with the genotyping of a single target ${ }^{18,43}$. In addition, some of these other variants may actually be the causal variant(s), simply transferring a disease association to the target multi-allelic gene through $\mathrm{LD}$, a particularly serious concern for the HLA-located $C 4$ gene $27,43,65,68,91,92$, a case where high LD to a plethora of SNPs and CNVs makes it difficult to pinpoint a single variant as causal.

On a positive note, SNPs in the vicinity of mCNVs may offer substantial information that is currently unexploited. SNP genotyping is a mature, reliable technology that has already been applied to millions of genomes ${ }^{93,94}$. While an individual SNP cannot serve as a proxy for a multi-allelic variant, it is nonetheless likely that the individual structural alleles of an mCNV arose on specific SNP haplotypes. Depending on the mutation rate of the $\mathrm{mCNV}$, as well as the age and number of alleles, the structural alleles may continue to bear relationships to those SNPs, and those SNPs may be used to inform on the copy number or allelic make-up of an mCNV in an individual.

\section{The rationale of the dissertation}

With the current controversies revolving around an inability to replicate $\mathrm{mCNV}$ associations, I wanted to investigate the unique challenges of associating mCNVs to phenotypes and to propose a study model that would yield replicable results. I hypothesized that this could be achieved by first obtaining precise copy numbers with multiple genotyping methods (to ensure the accuracy of the copy numbers), then characterizing the alleles of the mCNV and their relationship to SNPs and SNP haplotypes, allowing me to exploit that information when testing for associations. With these technical genotyping methods (measuring copy number) and theoretical models 
(analyzing structural and SNP haplotypes) working towards the same goal of testing a locus for an association, the study would most likely be resistant to the confounds affecting a single method of experimentation and would produce replicable results.

\section{The amylase locus}

In order to investigate the challenges of associating mCNVs to phenotypes, I needed to select a model $\mathrm{mCNV}$ that had a range of copy numbers and a likely association to a phenotype. The amylase locus (Figure I-3) in humans contains the three related genes responsible for digesting starch into sugar and also contains inversions, deletions, and duplications ${ }^{95}$. Each of the three amylase genes varies in copy number, with $A M Y 1$ varying from $2-17$ copies $^{18,96}, A M Y 2 A$ from $0-8^{96}$, and $A M Y 2 B$ from 2-6 (as I report below).

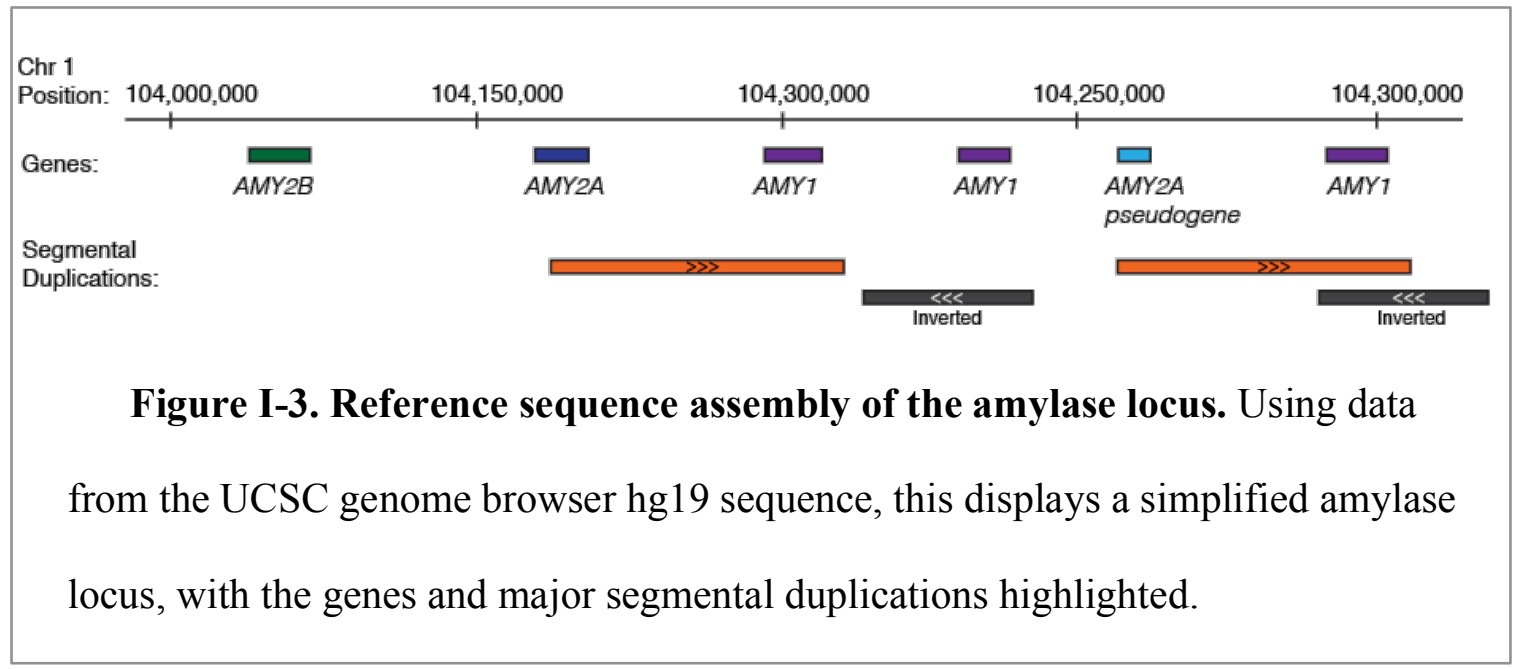

\section{Protein structure}

The AMY1 protein has 496 amino-acid residues, 1 calcium ion, 1 chloride ion, and 170 water molecules (Figure I-4). It folds into a structure consisting of three 
domains, A, B, and C. Domain A has a beta/alpha(8) barrel structure; it holds the chloride ion and contains the active site and catalytic residues. Domain B has no definite topology, but it holds the critical calcium ion. Domain C has a Greek-key barrel structure ${ }^{97}$, most likely plays a role in secretion, and contains glycosylation sites ${ }^{97-99}$. During catalysis, a highly mobile glycine-rich loop near the chloride ion becomes ordered while binding the oligosaccharide and moves in toward the substrate ${ }^{100}$. While the kinetic data suggest that the mobile loop may be involved in assisting catalysis during the transition state, crystallographic data suggest that the loop may play a role in the release of the product from the active site ${ }^{100}$.

The role of the calcium ion is critical. It stabilizes the secondary and tertiary structure of the enzyme ${ }^{101}$. However, the role of the chloride ion in the function of amylase appears to be complicated. It is not critical like the calcium ion, but it allosterically activates the enzyme and modulates both maximal activity and $\mathrm{pH}$ optima ${ }^{102}$. The maximum hydrolysis rate without chloride is $10 x$ lower than the rate with chloride, and that max rate is centered at $6 \mathrm{pH}$, as opposed to 7 for enzymes with chloride ${ }^{103}$.

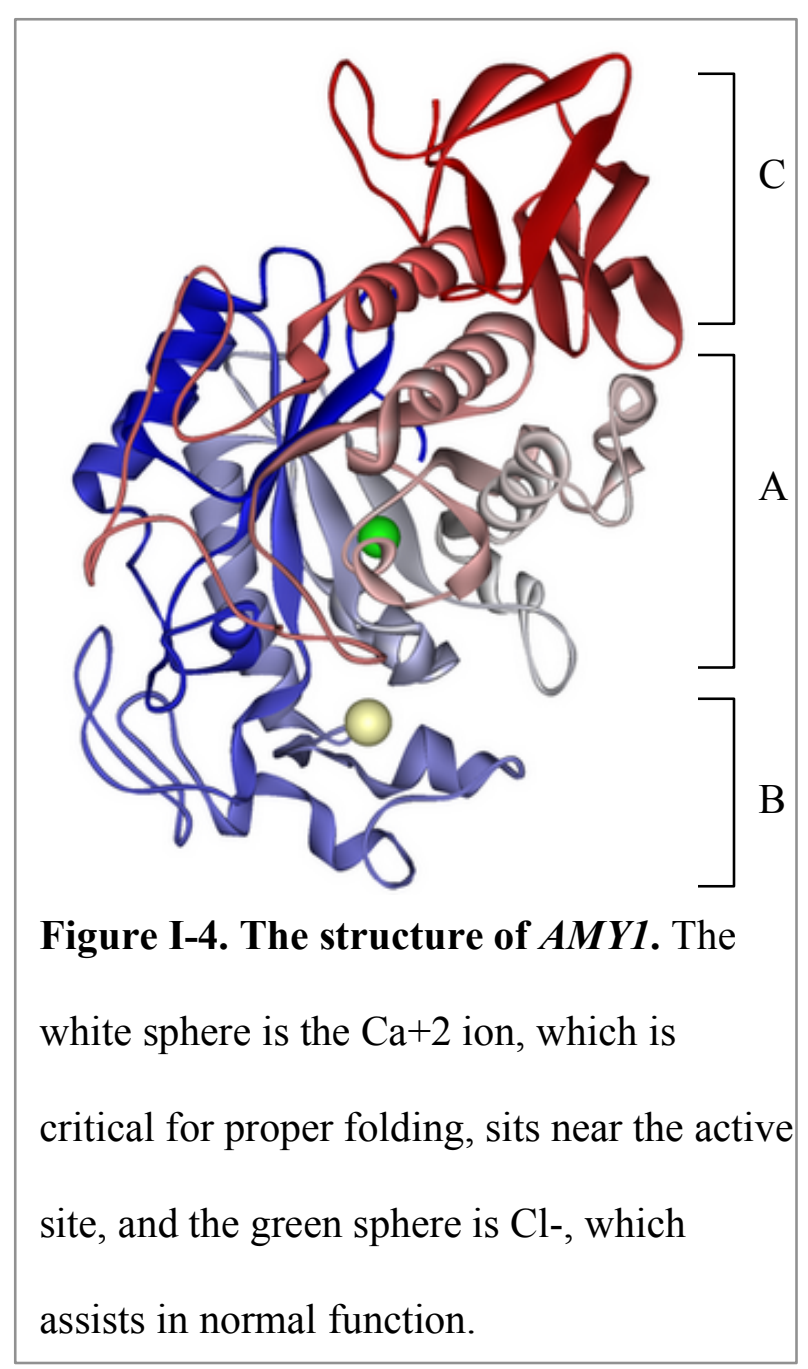


The pancreatic amylases, $A M Y 2 A$ and $A M Y 2 B$, share a nearly identical structure and $97 \%$ homology with $A M Y 1^{104}$. The known differences lie in post-translational modifications. Whereas some salivary amylase proteins are glycosylated ${ }^{98}$, very few of the amylase proteins from the pancreas are ${ }^{99}$, though it is not known why or what the function of the glycosylation is. In addition, the N-terminal glutamine residue of pancreatic amylases undergoes a post-translational modification to form a stable pyrrolidone derivative, presumably to protect the enzyme from digestion ${ }^{104}$.

\section{Function}

Amylases hydrolyze the 1,4-alpha-glucoside bonds in oligosaccharides and polysaccharides, thus catalyzing the first step in digestion of dietary starch and glycogen (Figure I-5). The human amylases tend to cut along the polysaccharides, producing maltose molecules ${ }^{105}$, but may also leave longer oligosaccharides. They do not degrade the polysaccharide entirely to glucose molecules, but a single mutation can activate this functionality ${ }^{106}$. Instead, they rely on other enzymes, such as maltase-glucoamylase, sucrase-isomaltase, and oligo-1,6-glucosidase, to completely degrade the dietary starch into glucose $\mathrm{e}^{107}$.

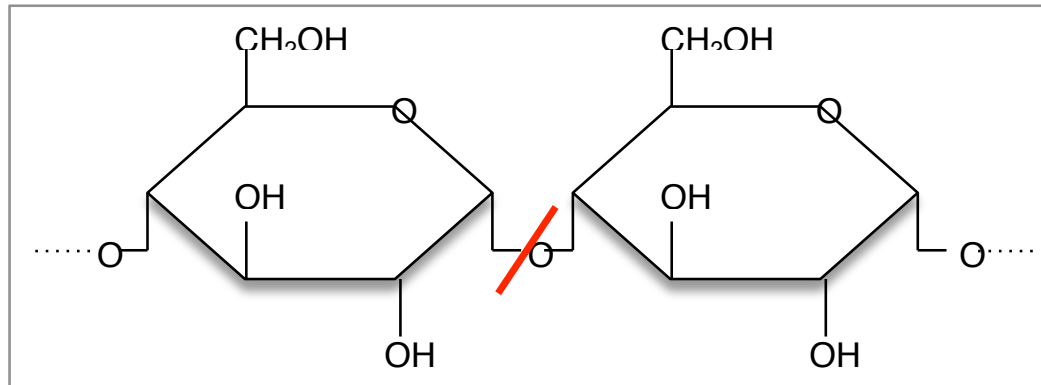

Figure I-5. The human amylases hydrolize the 1,4alpha-glucoside bond. Red line marks the cut site. They tend to degrade polysaccharides into maltose, though may leave longer oligosaccharides. 
Booming amylase expression is found only in the salivary gland (AMYl) and pancreas (mostly $A M Y 2 A$ and $A M Y 2 B$ ), though amylases can also be found in the serum, thyroid, liver, lung, and the female genital tract ${ }^{108-112} . A M Y 1$ is expressed in the salivary acinar cells, where it gets packaged in secretory granules and released into the acinar lumen along with MUC5B, MUC7, aPRP, bPRP, DMBT, Cystatin, Histatin, Statherin, LYZ, LPO, LL-37, electrolytes, and water ${ }^{107}$. Salivary amylase digests the polysaccharides in the oral cavity, both in solution and bound to the bacteria that constitute plaque ${ }^{113}$.

AMY1 is swallowed with the food and some of it is likely destroyed in the stomach, but with a sufficient amount of starch protecting it, up to 50\% of the AMY1 proteins are predicted to survive through to the small intestine ${ }^{114}$. Even in the absence of AMY1, which some newborns naturally and briefly lack $^{115}$, the pancreatic amylases can digest the dietary starch in the small intestines. In a pathway similar to AMY1, AMY2A and AMY2B are produced in pancreatic acinar cells and packaged into zymogen granules, which are later released into the small intestine ${ }^{107}$.

\section{Phenotypic Effects}

In the past, $A M Y 1$ was mostly used as a marker for stress. Increased AMY1 amount and activity correlates with cortisol and has been used to measure stress in burn victims, new parents, students, and psychiatric patients ${ }^{116-122}$. Though it is usually associated with stress, it would perhaps be more correct to state that it associates with

arousal, as good emotions can trigger $A M Y 1$ expression as much as bad emotions ${ }^{123}$, and exercise can also increase expression ${ }^{124}$. 
In addition to functioning as a marker for arousal, amylase has also been investigated for being a marker for cancer. Chromophobe renal cell carcinoma nearly always has deletions in the $1 \mathrm{p} 21.1$ region encompassing the amylase genes ${ }^{125}$. In addition, $A M Y 2 A$ was identified as a possible tumor-suppressor gene in gastric carcinoma $^{126}$, and autoantibodies against AMY2A are present in cases of pancreatitis and fulminant type 1 diabetes $^{127}$.

Amylase specifically binds with high affinity to several oral commensal streptococcal species, including Streptococcus gordonii, Streptococcus mitis, Streptococcus parasanguinis, Streptococcus cristatus, and Streptococcus salivarius ${ }^{128-131}$. Amylase-binding bacteria constitute a substantial proportion of the total cultivable flora on human teeth and only colonize the mouths of animals with salivary amylase activity $^{128}$. Amylase binding facilitates biofilm growth in vitro, though the addition of saliva inhibits this growth ${ }^{113}$. If amylase interacts with microbes in the mouth, it may also interact with microbes in other areas that express it such as the intestines or female genital tract. Indeed, in the mucosa of the genital tract, amylase breaks down glycogen, allowing colonization by Lactobacillus ${ }^{132}$.

AMY1 copy number associates with ancestral diet; populations that have eaten starch-based diets having more copies of $A M Y 1^{18}$. This implies that $A M Y 1$ copy number may affect phenotypes related to ingesting starch. Swallowing food without chewing, thus giving AMY1 less time and surface area to work, reduces the glycemic response food $^{133}$. Conversely, people who are classified as having more amylase activity than average in saliva have lower blood glucose concentrations after eating, which the study authors interpreted as meaning those individuals are better adapted for ingesting $\operatorname{starch}^{134}$. 
Variation in blood glucose levels could affect the propensity for diabetes, and it has been reported that T2D-associated end-stage renal disease (ESRD) in African Americans is significantly associated with a CNV encompassing $A M Y 2 A$ and $A M Y 2 B^{135}$. It is also possible that AMY1 could influence diabetes or obesity through its action in the mouth. It has already been shown to alter the oral perception of starch viscosity ${ }^{136}$ and may contribute to how much or little a person eats.

The majority of the studies above were conducted by measuring amylase activity or protein amount. Amylase activity, though most likely more biologically relevant than pure copy number, can fluctuate based on arousal, circadian rhythms, diet, and the time of the most recent meal ${ }^{137}$. Despite the environmental influences, $A M Y 1$ copy number correlates to AMY1 amount with an $\mathrm{r}^{2}$ of $0.35-0.5^{18,28,136}$, and across time and conditions, AMY1 amount is relatively robust and stable ${ }^{117}$.

If amylase copy number does affect a phenotype, it would be a tempting therapeutic target because it is present in an easily accessible part of the body (digestive tract) and has known inhibitors ${ }^{138}$. Amylase inhibitors appear to have some efficacy in reducing the glycemic response to food ${ }^{139}$ and assisting with weight loss ${ }^{140}$. Some of these inhibitors are easy to purchase and can mix easily with food ${ }^{140}$, which can create popular, yet dangerous, diet crazes - the most well known of which is the white kidney bean incident in Japan, where a weight loss TV program advertised eating food with toasted white kidney beans on it (known to contain an amylase inhibitor). This resulted in 100 hospitalizations for lectin poisoning ${ }^{141}$.

The poisoning was unrelated to inhibiting amylase. Indeed, there is no known phenotype associated with missing amylase activity. A family with a total deletion in 
$A M Y 2 A$ has been found, but it was not associated with any phenotype ${ }^{142}$. On a population-scale, some newborns do not express $A M Y 1$ until one month of age and most do not express it at near adult levels until three months of age, with the analyzed newborns displaying no difference in growth, weight, or tolerance of starchy foods ${ }^{115}$. This indicates that there is likely enough redundancy in the function of the amylase genes to create a robust network that can withstand the deletion of one type. On the other hand, with no reported instances of a complete and life-long depletion in $A M Y 1$ or $A M Y 2 B$, it is possible that these could be embryonic lethal or under extreme negative selection.

\section{Evolution}

Though most eukaryotes contain amylase genes ${ }^{143}$, the origin and organization of humans' $A M Y 2 B, A M Y 2 A$, and $A M Y 1$ gene set is found only in the apes ${ }^{144}$. Though it was the last to be discovered in humans, $A M Y 2 B$ is actually the ancestral amylase gene that is shared among mammals, and perhaps, even shared with fungi ${ }^{104} . A M Y 2 B$-only is found in New World and Old World Monkeys, though Old World Monkey express $A M Y 2 B$ in saliva, due to an unknown mechanism ${ }^{144}$ (Figure I-6). 


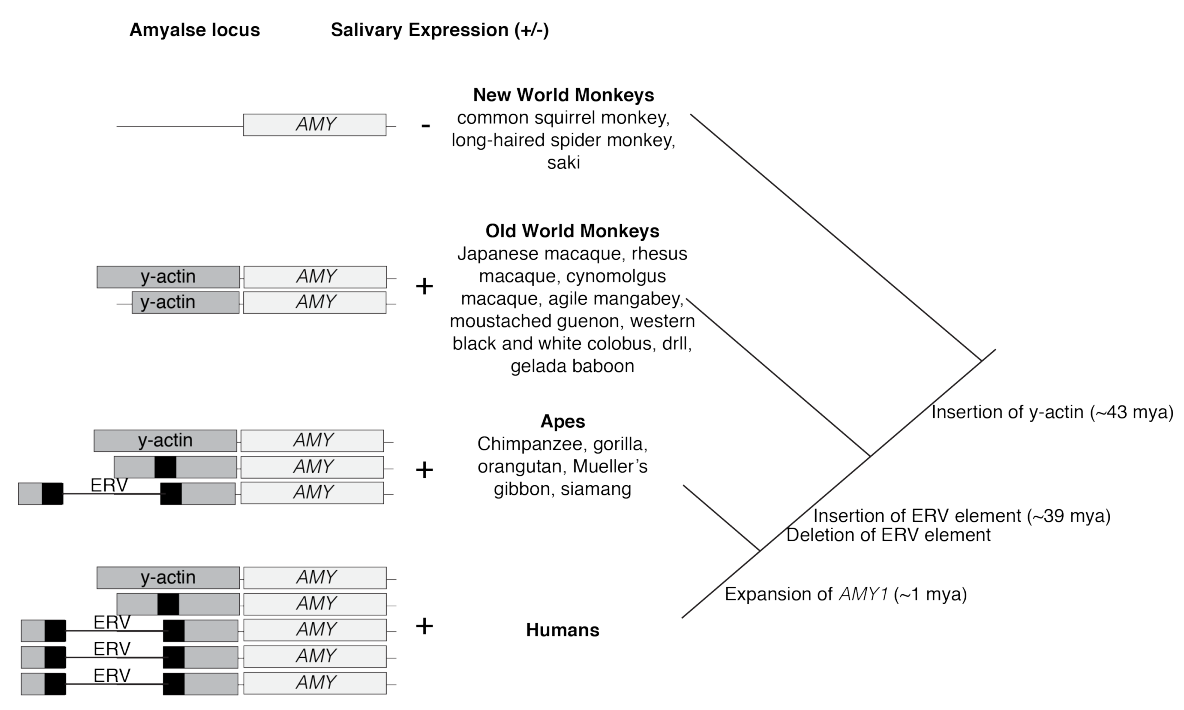

Figure I-6. Amylase promoters of the primates. $A M Y 1$ gained expression in saliva from an ERV insertion. Apes and humans share the same amylase genes, whereas New and Old World Monkeys have the ancestral genes. Old World Monkeys have expression in the saliva caused by an unknown mechanism. The species that have been analyzed are listed below the classifications.

In the common ancestor of the apes, $A M Y 2 B$ duplicated to create a progenitor $A M Y 1$, and soon after, an endogenous retroviral element (ERV) inserted into the promoter region, conferring salivary expression and creating the modern $A M Y 1$ gene. Then, $A M Y 1$ duplicated into a progenitor $A M Y 2 A$, which soon deleted most of the ERV in its promoter by recombining the ERV's LTR regions - thus forming the modern $A M Y 2 A^{144}$. About a million years ago, after the divergence from apes, $A M Y 1$ began to duplicate, creating an array of copy numbers in modern humans ${ }^{144}$ (Figure I-7). As evidenced by my research, $A M Y 2 B$ and $A M Y 2 A$ also duplicated, but to a lesser extent. 


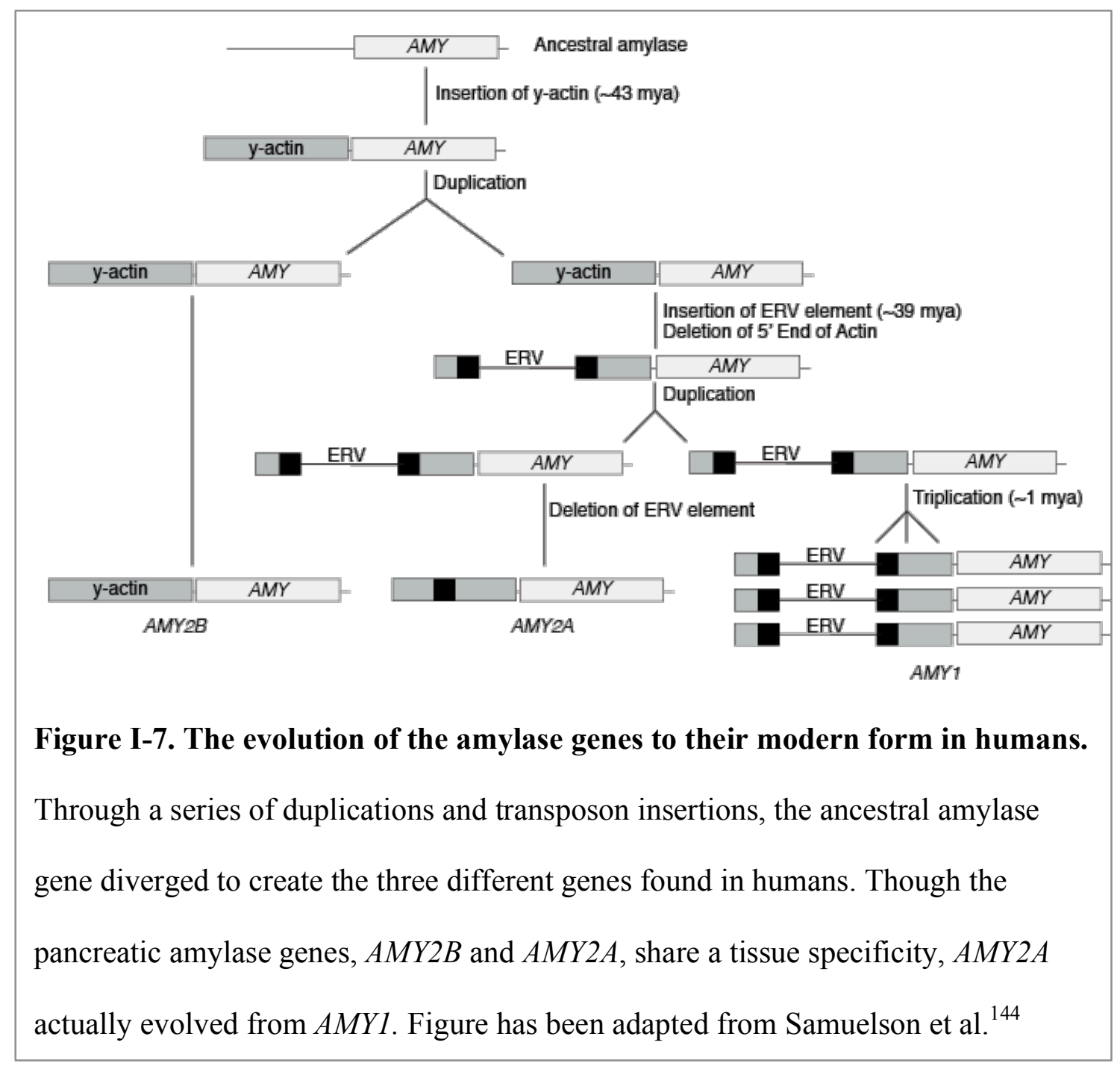

Non-allelic homologous recombination (NAHR) is commonly assumed to be the mutational mechanism behind these duplications ${ }^{145}$, and models based on NAHR exist that could explain how the ancestral $A M Y 2 B-A M Y 2 A-A M Y 1$ cassette mutated into the human reference sequence's $A M Y 2 B-A M Y 2 A-A M Y 1-A M Y 1-A M Y 2 A$ pseudo-AMY1 cassette $^{145}$ (Figure I-8). However, multiple NAHR steps would have to occur over several generations without leaving any apparent intermediate haplotypes in the modern populations. 
Another likely mutational mechanism could be the set of replication error mechanisms, FoSTes/MMBIR (fork stalling and template switching/microhomologymediated break-induced replication). In these mechanisms, the DNA replication fork either stalls or collapses and an extending strand invades another replication bubble to continue synthesis for a brief time ${ }^{146}$. Unlike NAHR, these mechanisms can result in inversions and only rely on microhomology. However, with only one of the $A M Y 1$ genes inverted, it is difficult to imagine how a mechanism such as this could produce the human reference cassette with only one mutational event, though one is suggested in Figure I-9.

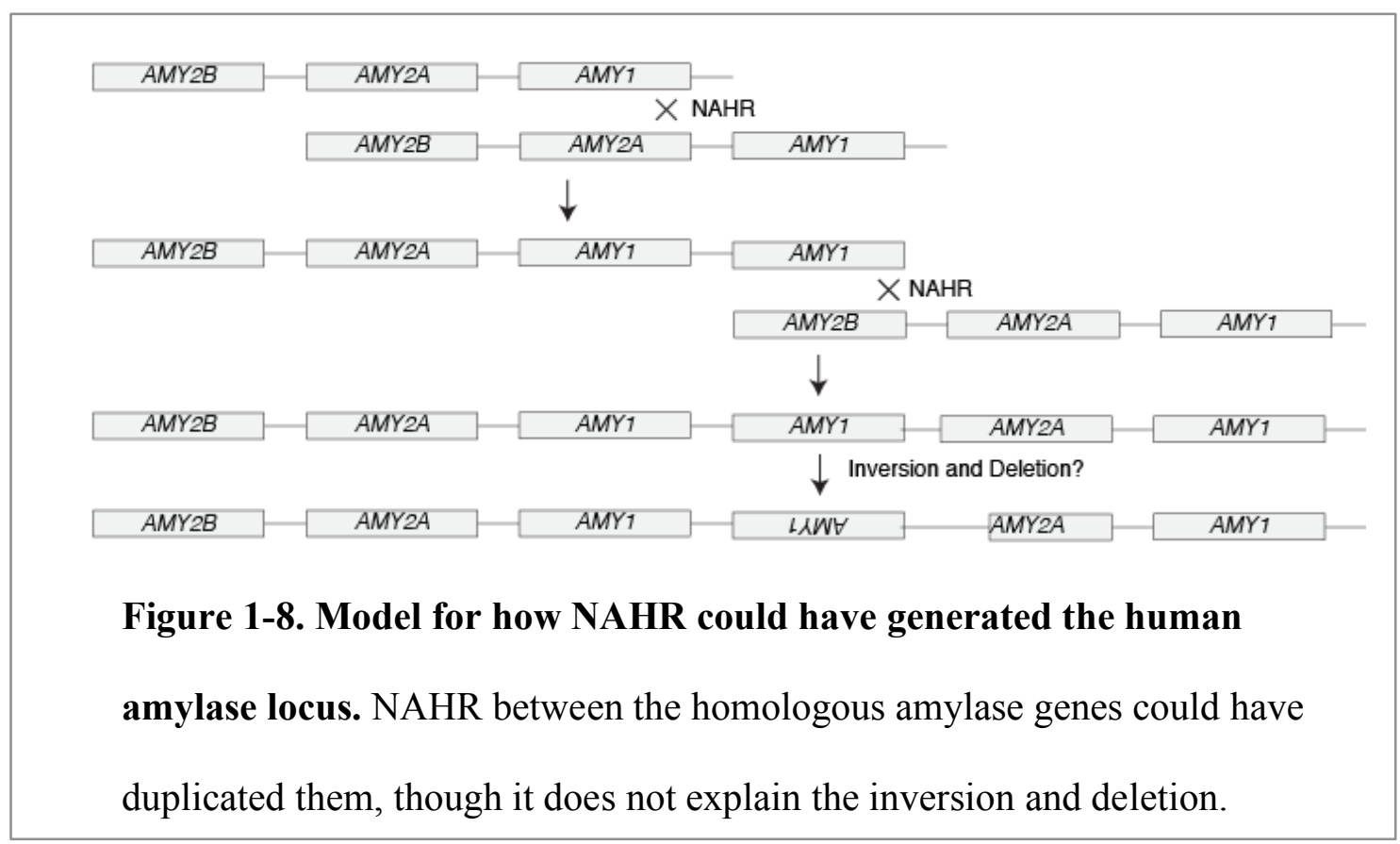




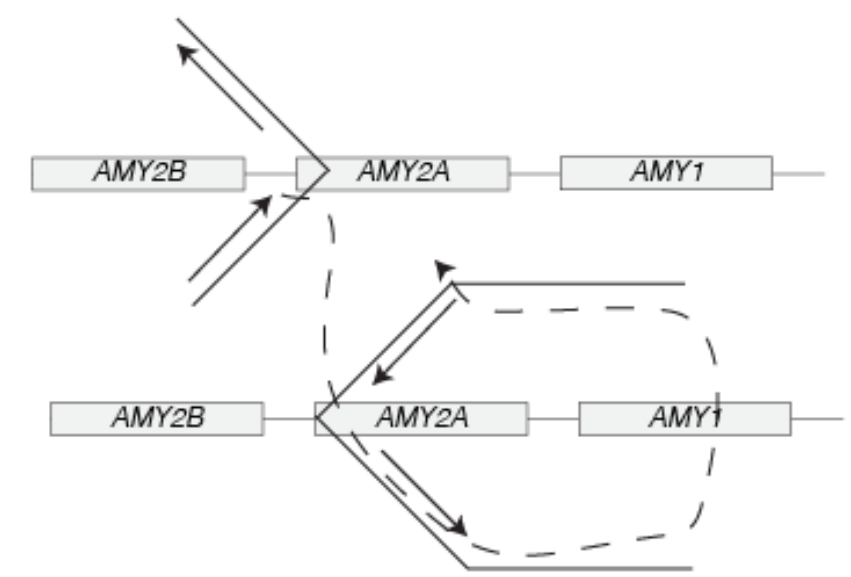

Figure I-9. Possible replication fork-linked mutational mechanism.

If the replication fork (marked by solid lines in the shape of a V) stalled or collapsed, the leading strand (dotted line) may invade a nearby replication bubble, copy the $A M Y 2 A$ and $A M Y 1$, switch to the other side of the bubble to copy an inverted $A M Y 1$ before the original fork restarts to finish copying part of the $A M Y 2 A$ and all the $A M Y 1$.

Humans are not the only species to have had duplication events at the amylase locus. Dogs also have duplicated $A M Y 2 B$, resulting in up to 30 copies in a single genome $^{147}$. In addition, other proteins in the dog starch metabolism pathway, a maltase and a glucose transporter, are under positive selection. Dogs do not express amylase in their saliva, so these other adaptations may have been necessary to cope with a starchrich human-influenced $\operatorname{diet}^{147}$. Mice have also duplicated their $A M Y 2 B$ into two versions: one expressed in the pancreas and the other in the salivary glands ${ }^{148}$. Coincidentally, the 4 or 5 pancreatic amylase genes are named Amy $2 a 1-5$, the salivary amylase is $A m y 1$, and a pseudogene is named $A m y 2 b^{149,150}$ (Figure I-10). There are indications that the copy 
numbers of these genes may vary across strains ${ }^{151,152}$. In addition, there is direct and indirect evidence that salivary amylase is duplicated in other species such as bonobos, gorillas, and orangutans ${ }^{18,153}$.

As such, I selected the amylase locus for its variation in copy numbers and possible phenotypic impact.

Chr 3 Position:
Genes:
Segmental
Duplications:
Figure I-10. The
and psuedogenes like the human locus, it does not resemble the pattern of the human
locus.




\section{Chapter 1. Measuring the amylase locus}

In order to investigate the challenges of associating mCNVs to phenotypes, I needed to select a model mCNV that had a range of copy numbers and a likely association to a phenotype. The amylase locus contains the three related genes responsible for digesting starch into sugar and also contains inversions, deletions, and duplications ${ }^{95}$. Each of the three amylase genes varies in copy number, with $A M Y 1$ varying from 2-17 copies $^{18,96}, A M Y 2 A$ from $0-8^{96}$, and $A M Y 2 B$ from 2-6 (as I report here).

Given their role in starch metabolism and $A M Y 1$ 's greater average copy number in three populations with high starch $\operatorname{diets}^{18}$, it was long hypothesized that $A M Y 1$ copy number plays a role in dietary and metabolic disorders, such as obesity and diabetes, but at the start of this study, nothing convincing had yet been reported (see Chapter $\mathbf{4}$ for the recently reported association).

After selecting the amylase locus as a testing ground, I wanted to eventually perform an association. Yet, first I needed to be able to accurately measure copy number, preferably in such a way that I could definitevely call an integer copy number for most 
individuals. Southern blot and qPCR have both been used previously at this locus, but Southern blot is not high-throughput, and qPCR gives imprecise $A M Y 1$ copy numbers that form a bell-shaped distribution ${ }^{18,95}$. I decided to use droplet digital PCR $(\mathrm{ddPCR})^{154,155}$ and Genome STRiP ${ }^{16,156}$, which have been used to precisely measure copy number at other loci in large sample sizes ${ }^{16,21}$.

In short, ddPCR can be thought of as a qPCR that has been emulsified into droplets. After amplification, the droplets that contain the target sequences are fluorescent, due to the action of the TaqMan assays, and the number of fluorescent droplets is counted. In rough terms, one fluorescent droplet represents one target sequence that was present in the $20-\mu l$ reaction. Thus, ddPCR gives a near-exact estimate of the number of target and control sequences, which can be calculated back to a CNV copy number. The advantage of ddPCR over qPCR lies in the fact that ddPCR counts target and control sequences (as opposed to comparing PCR curves) and does so after amplification (which reduces the effect of differential PCR efficiencies between the control and target regions).

Despite the advantages of ddPCR, it still requires access to $\sim 20 \mathrm{ng}$ of quality DNA. Genome STRiP, a computational method that can determine copy number from sequencing data, can increase the number of known-copy number individuals by utilizing public sequencing databases. This algorithm counts how many sequencing reads align to a target region, compared to those aligning elsewhere. Regions containing more reads than the genome-wide average are called as duplications, whereas those that have less are deletions. This change is quantitative and can be translated into a copy number measurement at the specified locus. This method has the advantage of genotyping any 
particular locus relatively quickly (after the sequencing has already been done) and allows for genotyping refinement, in that certain parameters of the algorithms can be adjusted and optimized until the copy numbers cluster at integers.

While Robert Handsaker, the creator of Genome STRiP, used his program to measure amylase gene copy number in 569 individuals sequenced in Phase 1 of the 1000 Genomes Project ${ }^{157}$, I used ddPCR to genotype an overlapping set of individuals: 114 parent-offspring trios from HapMap ${ }^{158}$. This parallel effort allowed us to separately investigate copy number and to draw our own conclusions - if our conclusions matched, then there was a higher likelihood of our findings being correct.

\section{Optimizing the measurement of $A M Y 1$}

Though ddPCR has worked well on other loci, those loci tended to have fewer than 6 copies $^{16,21}$ (and unpublished findings). AMY1 has an average of 6 copies and can have as many as 17 , which requires an increased level of sensitivity and precision. Using ddPCR worked well for genotyping $A M Y 2 A$ and $A M Y 2 B$, but $A M Y 1$ initially gave poor genotypes and, thus, required an extensive amount of optimization, as described below. 
(1) The AMYl assay: Normally, qPCR assays function the same or better in a ddPCR reaction (unpublished data). However, using a previously published qPCR assay ${ }^{18}$ failed to give tight, separate clusters of target-positive, control-positive, and doublepositive droplets (Figure 1-1). Additionally, the assay failed to give copy number measurements that clustered at integers in both ddPCR and $\mathrm{qPCR}^{18}$. I ordered and screened four custom assays that were predicted to only bind within the $A M Y 1$ gene (as opposed to within $A M Y 2 B$ or $A M Y 2 A$, based on Clustal analysis ${ }^{159}$ ), to amplify efficiently (based on Primer 3 analysis ${ }^{160}$ ), and to avoid SNPs (UCSC Genome Browser $\left.{ }^{149}\right)$. This group yielded two assays, AMY1_assay1 and AMY1_assay2 (Appendix B), that produced tight droplet clusters (Figure 1-2) and copy numbers that were closer to integers, though more optimization was needed.

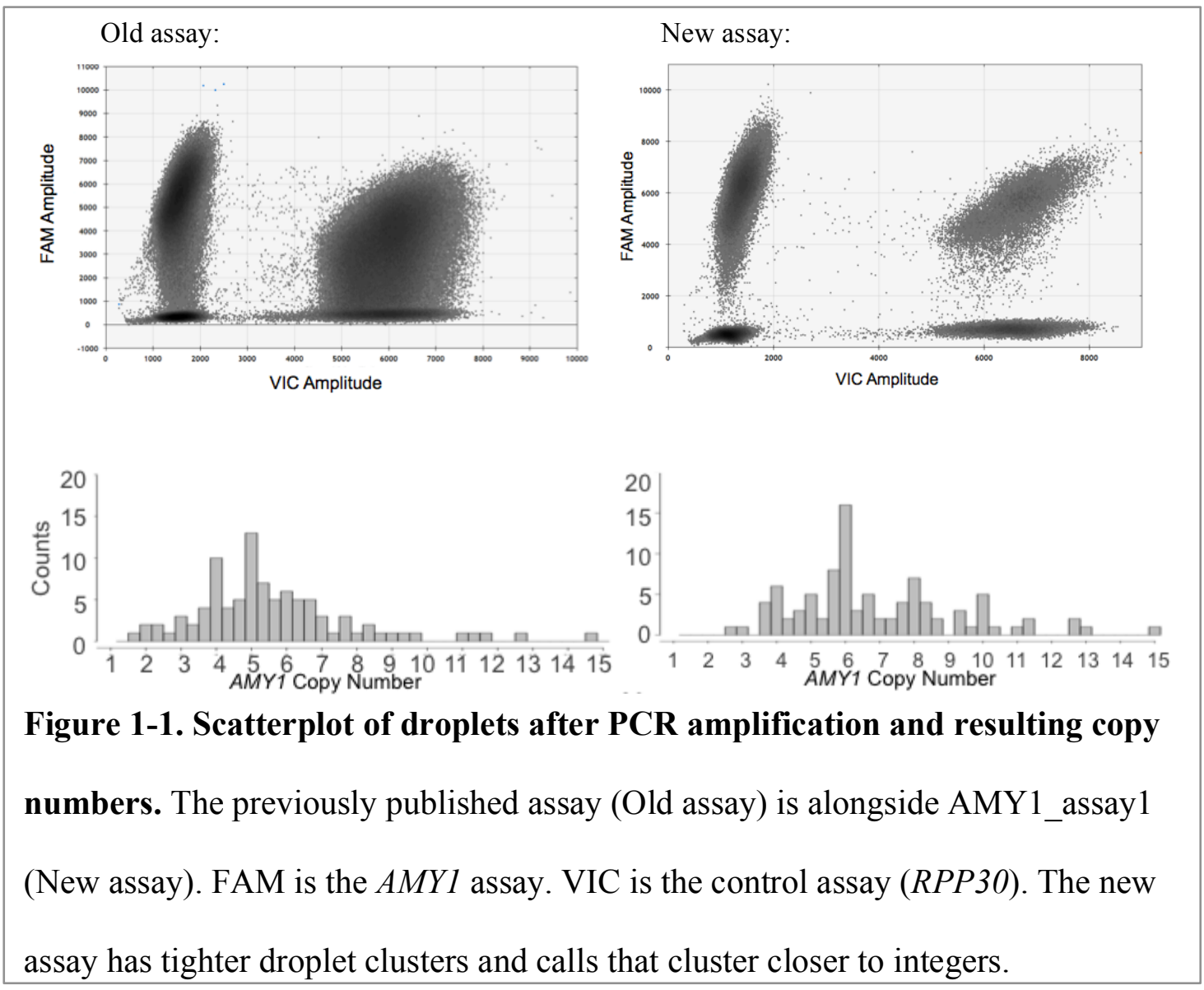



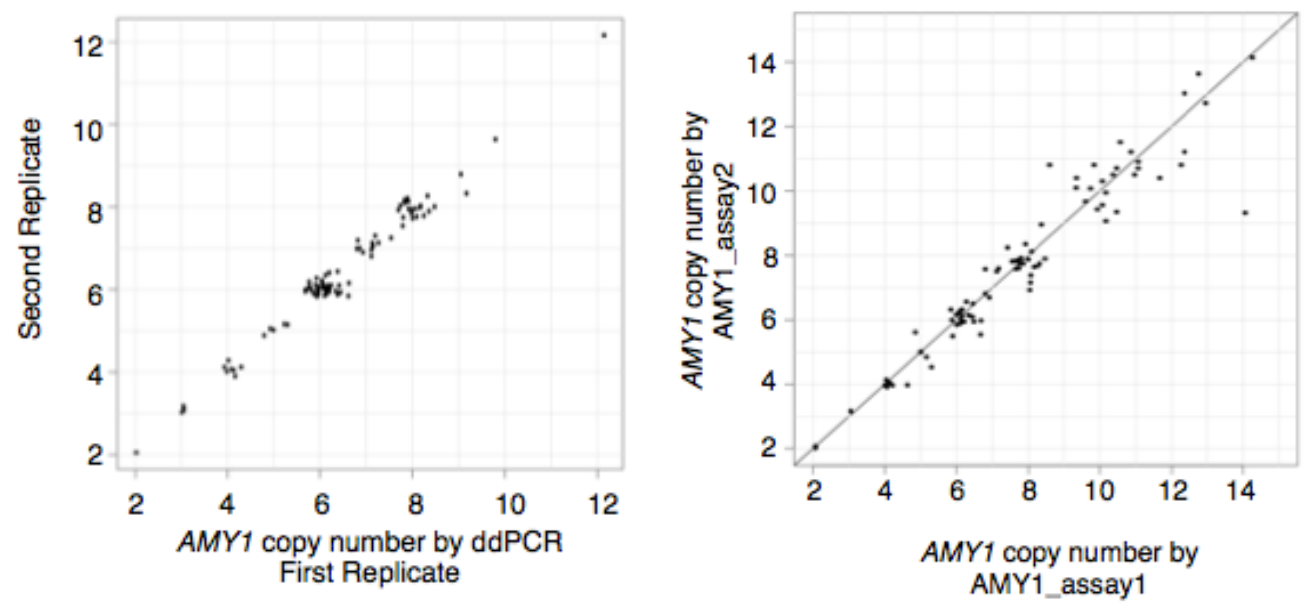

Figure 1-2. Replicability of $A M Y 1$ copy number between technical replicates and different assays. For the most part, $A M Y 1$ copy number is concordant across replicates, more so if the same assay is used, reflecting that there are assayspecific factors at play.

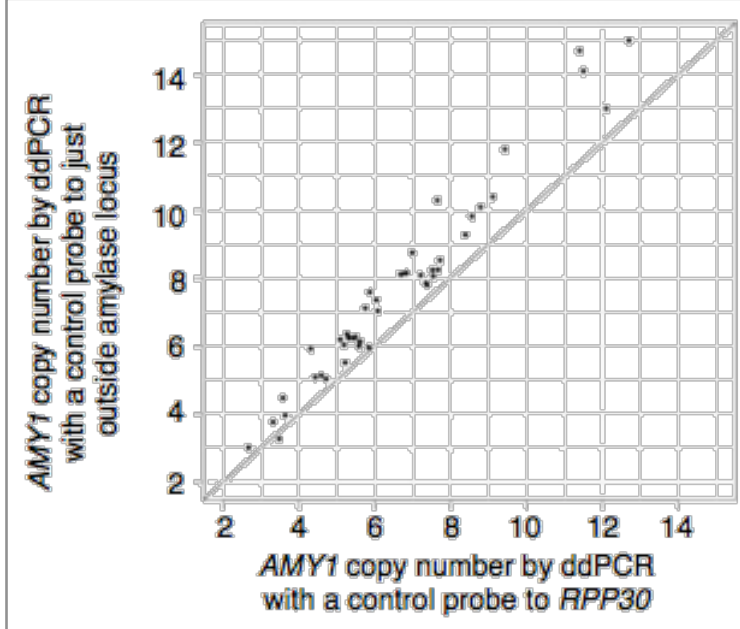

Figure 1-3. Effect of control assay on copy number call. Amylase is in a latereplicating region, whereas $R P P 30$ is not. This leads to calls that are $\sim 10 \%$ below the true value, as estimated by a replicationmatched control.
(2) The control assay: A standard commonly used in ddPCR is an assay to RNaseP Protein P30 (RPP30) ${ }^{154,155,}$ which is a housekeeping gene located in a genomic region that replicates early in the cell cycle ${ }^{161}$. However, since the amylase locus is in a late-replicating region, DNA isolated from replicating cells will naturally have less of it than $R P P 30$ (Figure 1-3). This artificially deflates the copy number calls by an 
amount corresponding to the percent of cells that had already replicated $R P P 30$, but not $A M Y 1$, at the time the DNA was extracted. Because the raw copy number calls using the $R P P 30$ probe are about $10 \%$ less than those with a replication-matched control, I can calculate the percent of cells caught between early and late replication at $\sim 20 \%$ of cells, though the exact number most likely varies by cell line. To counteract the replication effect, I used a control assay targeted to just outside the amylase region, referred to as Near_AMY (Appendix B).

(3) Batch effects: A plate-wide correction factor was applied to each plate to correct for batch effects. The factor was generally between -5 and $5 \%$, and was calculated by averaging the distance to the nearest integer for the best genotypes (the ones with a confidence interval range less than 0.75 and having more than $10 \%$ negative droplets, indicating the input DNA was at an optimal concentration). Because the same DNA samples needed different correction factors for different genotyping runs, the factor(s) causing the discrepancy are most likely technical and/or assay-specific.

(4) DNA concentration: The sometimes high copy numbers of $A M Y l$ can oversaturate the ddPCR reaction, resulting in an inaccurate genotype call. The likelihood of this happening correlates with the amount of input DNA and the $A M Y 1$ copy number; generally, many samples oversaturated at DNA concentrations greater than $5 \mathrm{ng} / \mu \mathrm{l}$ in the ddPCR reaction. Combined with the fact that samples in the same plate often vary in DNA concentration, one plate-wide ddPCR reaction nearly always resulted in some samples being oversaturated $(<10 \%$ double-negative droplets) and others undersaturated. Precise copy number calls could only be obtained by performing two reactions - one at a high sample concentration designed to ensure each sample has the 
minimum DNA input ( $1 \mathrm{ng} / \mu \mathrm{l}$ in the reaction) and one at a low sample concentration, to genotype those that oversaturated their first reaction (using a concentration that was determined by evaluating the first run).

(5) After our initial investigations into 1000 Genomes, we found that $A M Y 1$ copy number was only even when $A M Y 2 A$ copy number was even, and odd when $A M Y 2 A$ was odd (discussed below). This observation led to a genotyping pipeline where the $A M Y 2 B$ and $A M Y 2 A$ assays were done first to determine the concentrations of the samples (enabling the high-low AMY1 genotyping scheme discussed above). Then, if the two $A M Y 1$ runs disagreed on the genotype of a particular sample, the $A M Y 2 A$ call was used to decide which $A M Y 1$ call was correct (Figure 1-4).

In parallel, Robert Handsaker was optimizing Genome STRiP to work on the lowcoverage sequencing data of 1000 Genomes. He optimized the genotyping of amylase by specifying various ranges of base pairs as the "official" $A M Y 1$ duplicated region; certain ranges yielded better copy numbers than others, and the best range was used for genotyping. In this way, he defined to near-base pair resolution which segments of the amylase locus were commonly duplicated. Figure 1-5 displays these breakpoints for the GRCh37/hg19 human reference sequence. Figure 1-6 gives the unrounded copy number measurements for both ddPCR and Genome STRiP for the three amylase genes. Though there was still noise present in the measurements, the copy number calls clustered near integers and were concordant between both methods. 

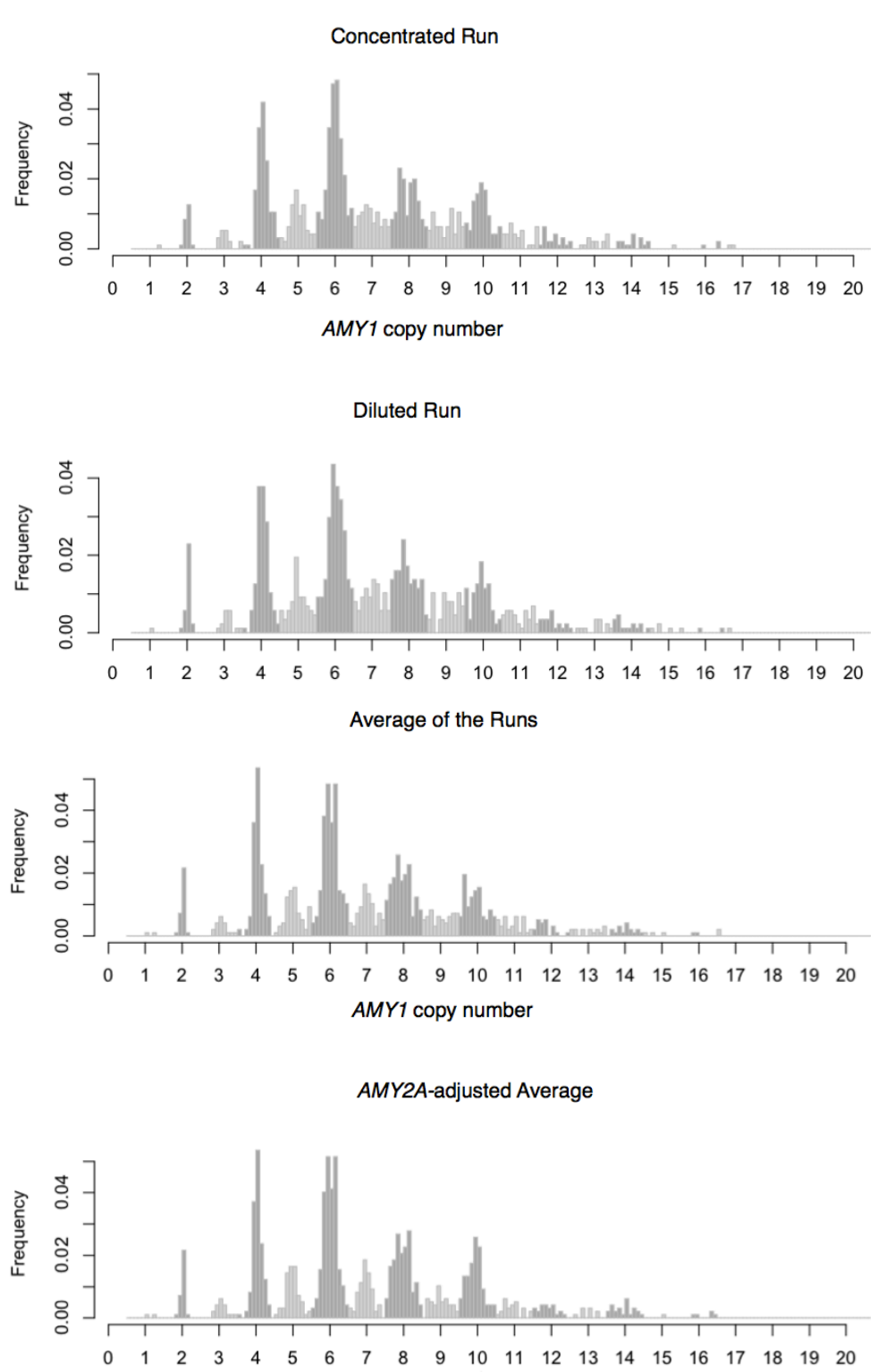

Figure 1-4. Copy number distribution during an $A M Y 1$ genotyping workflow.

The top two histograms are for the concentrated and diluted sample DNA concentration runs, respectively. The bottom two are the average of the runs and the $A M Y 2 A$-adjusted copy number, respectively. The bars are colored gray and white for easier viewing. Copy numbers are closer to integers when adjusting for $A M Y 2 A$, indicating that it screens out imprecise $A M Y 1$ copy number calls, whereas simple averaging allows those imprecise calls to distort the good calls. 


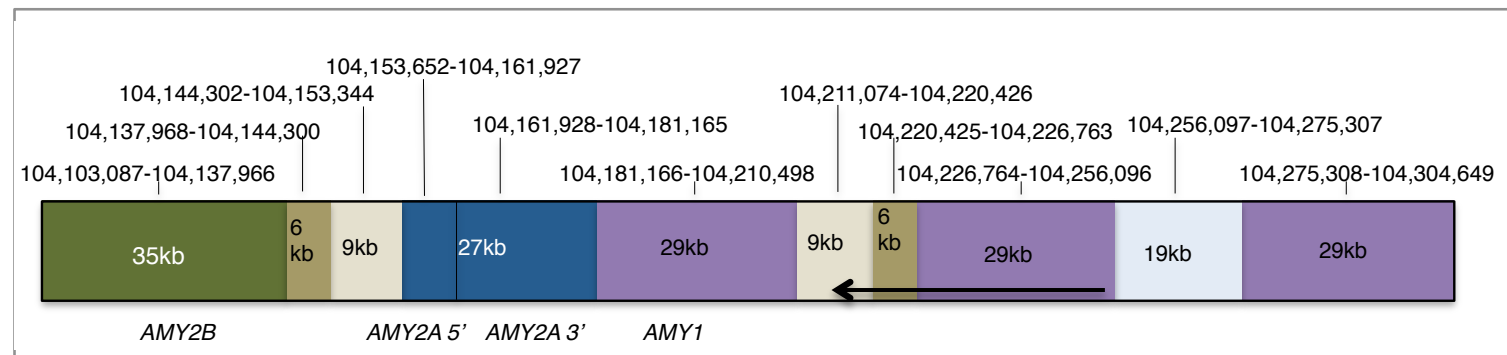

Figure 1-5. Reference sequence divided into the blocks of genetic sequence that vary in populations. The displayed numbers are the chromosome coordinates of the blocks. The brown segments are devoid of genes, but vary in complicated patterns (Chapter 2). In the haplotypes of Figure 1-8 these regions are condensed into one for simplicity.

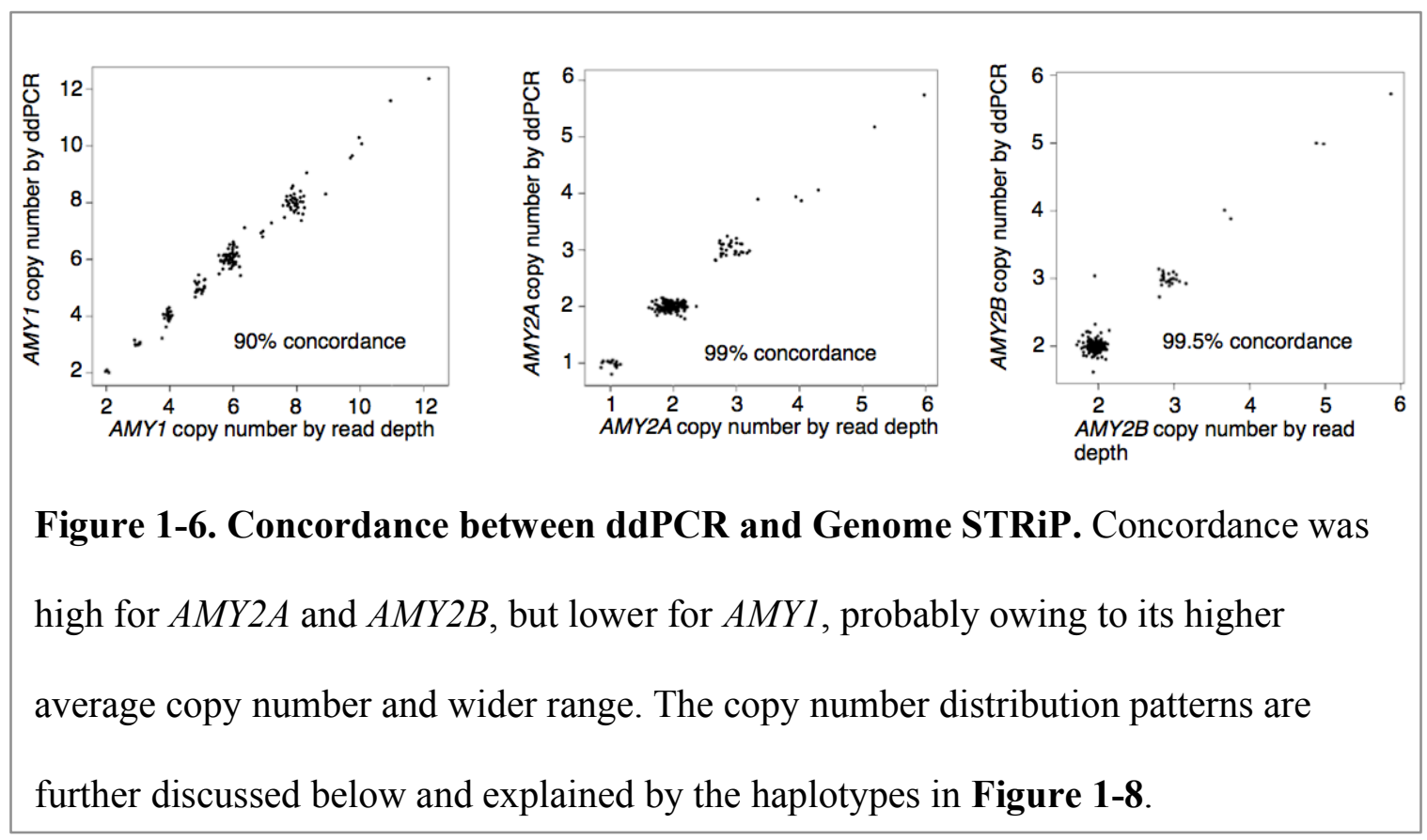

\section{The distribution of amylase gene copy number}

After optimization, we had copy number measurements for 569 individuals of the 1000 Genomes Project ${ }^{157}$ (covering three populations: European American, Chinese/Japanese, and West African) and an overlapping set of 114 European American 
and West African parent-offspring trios from HapMap ${ }^{158}$. These data, which were concordant across overlapping samples, revealed two novel relationships (Figure 1-7):

1) Individuals are four times more likely to have an even copy number $(2,4,6$, etc.) than an odd copy number $(1,3,5$, etc.) of $A M Y 1$.

2) $A M Y 1$ and $A M Y 2 A$ share parity - the copy numbers of $A M Y 1$ and $A M Y 2 A$ are almost always both odd, or both even.

These features - which we observed by two independent methods (ddPCR and Genome STRiP) - had not been reported in studies that used lower-precision methods, such as real-time qPCR or array CGH, to measure amylase gene copy number ${ }^{18,28,162}$.
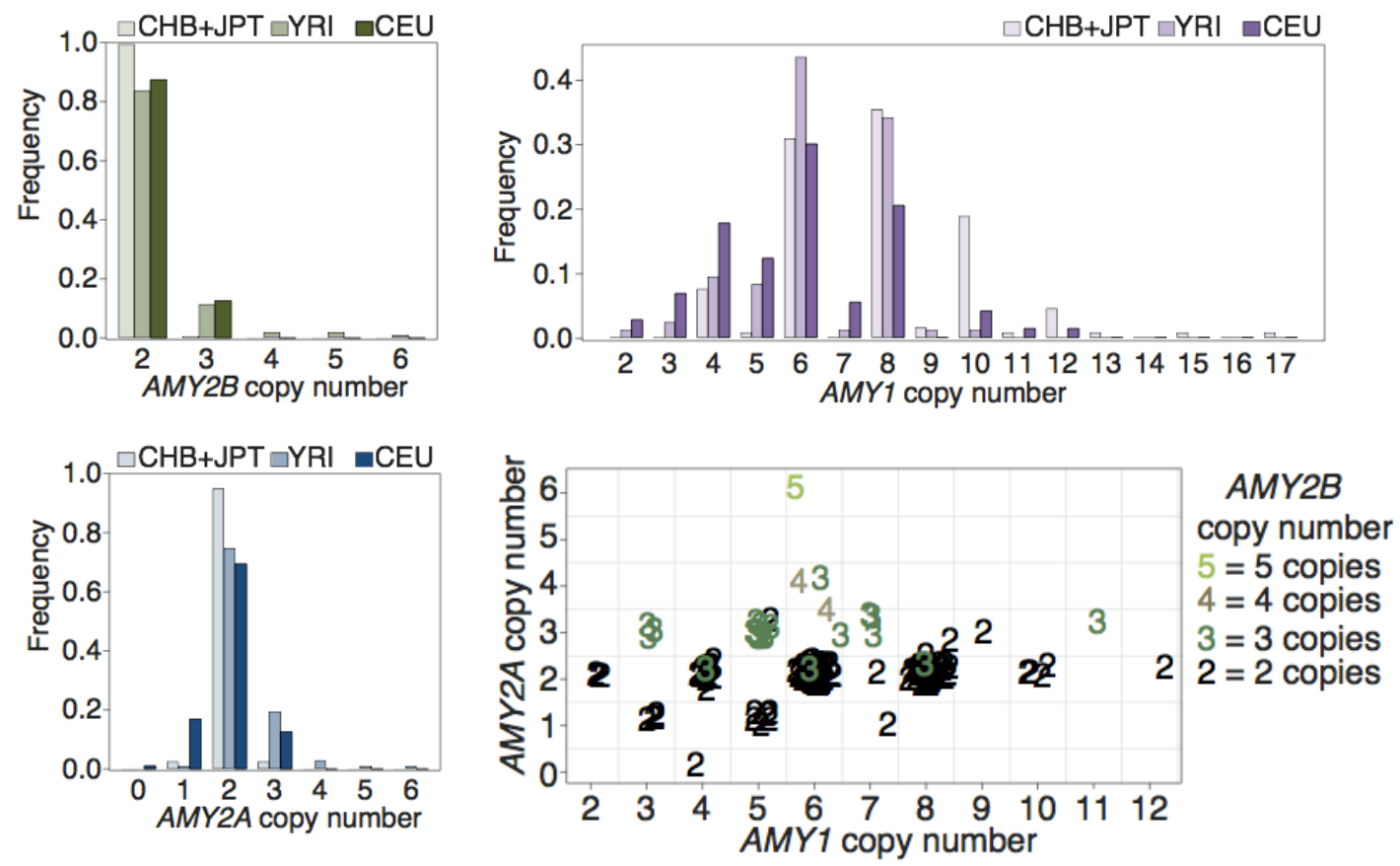

Figure 1-7. Population distributions of amylase gene copy number, and the relationship of the amylase genes to each other. Copy numbers are from Genome STRiP. Scatterplot uses only CEU and YRI data, because the JPT and CHB rarely have odd copy numbers. Chinese (CHB), Japanese (JPT), Yoruban (YRI), European American (CEU) 
These copy numbers must have arisen from an underlying set of structural haplotypes of the amylase locus. However, the known alleles could not account for these patterns, particularly the variation in $A M Y 2 B$ and $A M Y 2 A$ copy number ${ }^{18,95,145}$. I needed to characterize the novel structural haplotypes and confirm the known ones before I could investigate their relationships with each other and with the surrounding SNPs. Though I could technically perform a phenotype association study using only the copy numbers, I wanted to first investigate the haplotypes and alleles, because they could have held clues on how to better perform such an association study.

\section{The structural alleles of the amylase locus}

To determine the structural alleles of the amylase locus, I extended an approach used for the 17q21.31 locus, one of the first structurally complex loci to be resolved into a set of common structural haplotypes ${ }^{21,22}$. I followed the segregation of each amylase gene's copy number in the 114 father-mother-offspring trios and inferred the copy numbers of the amylase genes on the transmitted and untransmitted chromosomes (a process known as phasing) by comparing the copy numbers between parent and child. Assigning only the haplotypes that had been previously described ${ }^{18,95,145}$ to the chromosomes resulted in successful phasing for only $7 \%$ of the trios and $15 \%$ of individuals.

In the remaining unphased trios, there were patterns that could only be explained by novel haplotypes - in particular, patterns concerning duplications in $A M Y 2 B$ and partial deletions/duplications of $A M Y 2 A$ (Figure 1-8). When I added the three novel haplotypes that best explained the data to the phasing program, $98 \%$ of the 480 European-ancestry individuals in the 1000 Genomes Project had copy numbers that could 
be explained. The West Africans had additional, rare novel haplotypes that could not be fully identified with certainty, and when two of these haplotypes were added into the phasing program (totaling 10 haplotypes), $27 \%$ of trios and $39 \%$ of individuals, in both populations, could be successfully phased, with the vast majority of the remainder having copy numbers that were consistent with multiple combinations of haplotypes.

In summary, I inferred 8 common haplotypes consistent with 5 of the 6 haplotypes previously identified using cosmids ${ }^{95,145}$ and fiber $\mathrm{FISH}^{18}$, along with 3 novel haplotypes in the European American trios and evidence for additional, rarer haplotypes in the West African trios (Figure 1-9). To complete the list of haplotypes, I would need to know the allele frequencies. These frequencies could also provide clues about the evolution of the amylase locus.

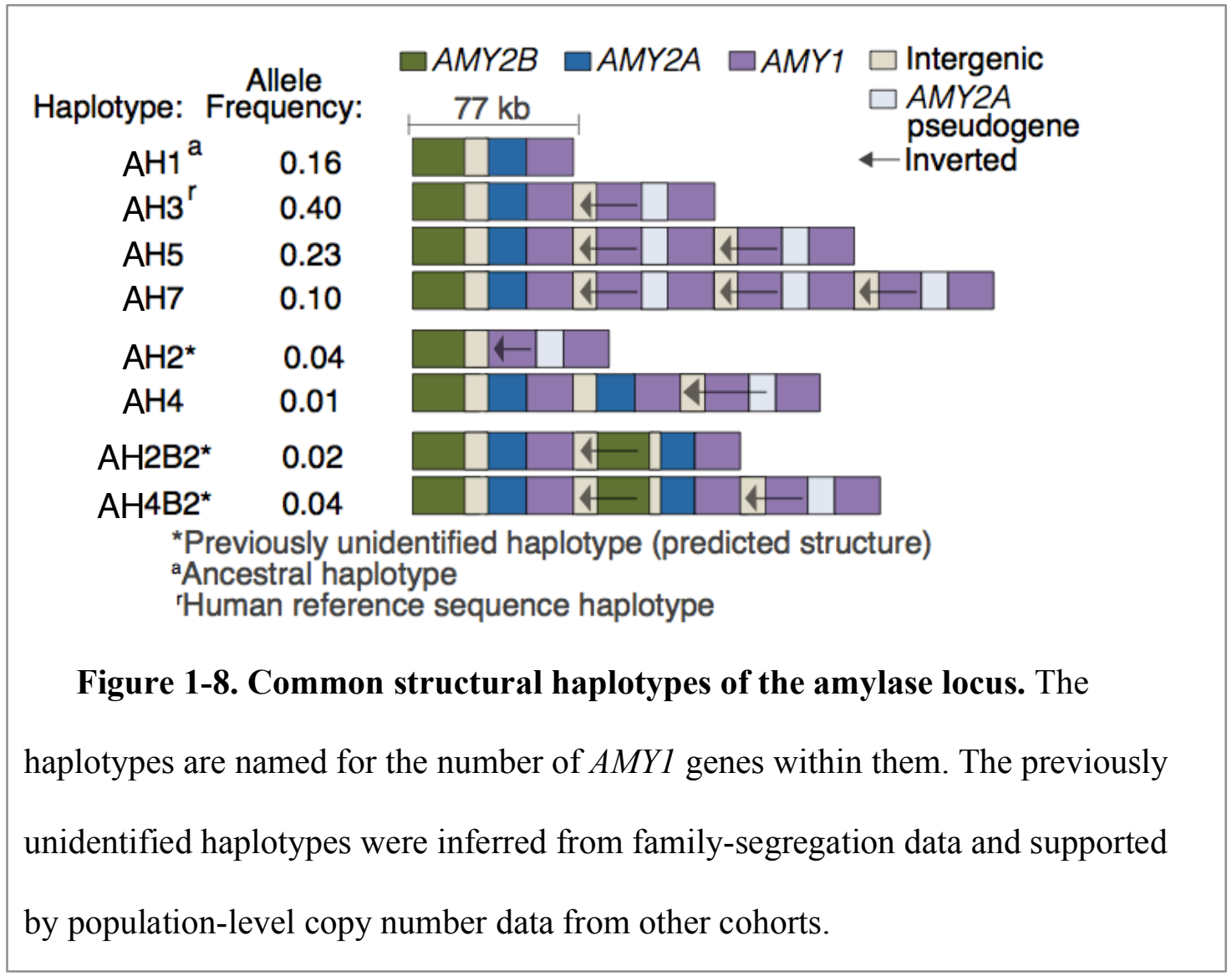



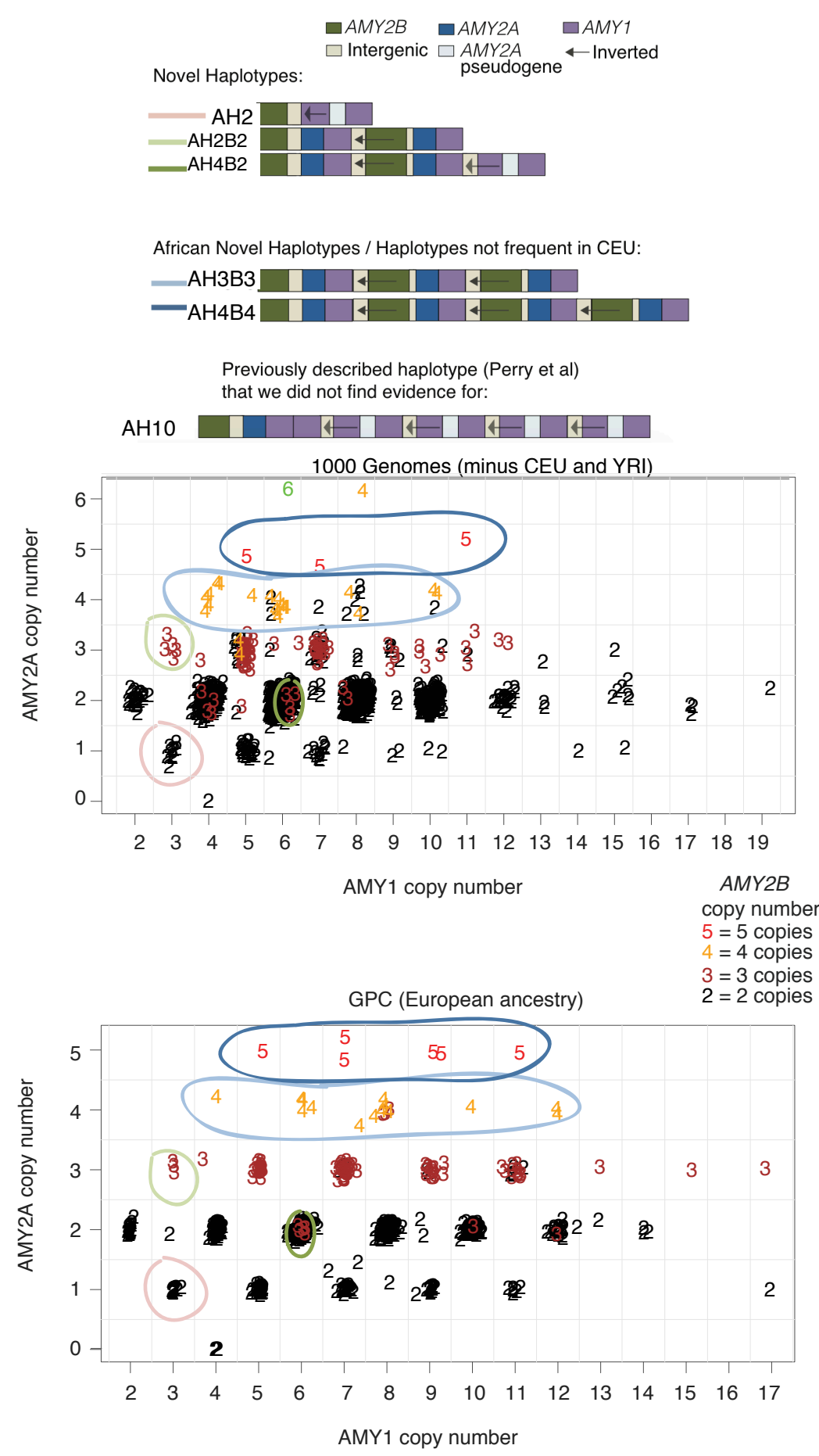

Figure 1-9. Evidence for novel haplotypes in other populations. The copy numbers of 1000 Genomes and the GPC cohort (768 European-ancestry individuals) are plotted. Evidence for each novel haplotype is circled in the corresponding color. The haplotypes also in Figure 1-8 were also found in family-segregation data. 
I could not calculate the allele frequencies of the haplotypes from the individuals I could phase, since this would artificially enrich for haplotypes that create unique, phaseable genotypes. Instead, I utilized the individuals carrying haplotype $\mathrm{AH} 2$, who can be identified because of their lower $A M Y 2 A$ copy number. I selected individuals carrying AH2 from the GPC cohort (768 European-ancestry individuals) and the European Americans of 1000 Genomes (142 individuals total) and identified their other haplotype by subtracting copy numbers. I calculated the frequency of each haplotype in this pool of other haplotypes. Unfortunately, the frequency of AH4 could not be determined this way, since it causes an increase in $A M Y 2 A$ that balances $\mathrm{AH} 2$ 's decrease. Instead, its frequency was determined by identifying individuals that carry AH4 (marked by an increase in $A M Y 2 A)$ in GPC and 1000 Genomes, and dividing it by the total.

One reason for characterizing the common haplotypes and their frequencies was to discover what was driving the patterns of $A M Y 1$ variation. The enrichment in diploid even copy numbers was caused by $89 \%$ of chromosomes carrying an odd number of $A M Y 1$ copies (referred to as $A M Y 1$-odd haplotypes). The parity between $A M Y 1$ and $A M Y 2 A$ was caused by the $A M Y 1$-odd haplotypes each having one copy of $A M Y 2 A$, while the $A M Y 1$-even haplotypes have either zero or two copies of $A M Y 2 A$.

Yet, I could not be sure of the order of genes on the haplotypes, which could help determine the evolutionary history of the locus and explain the presence or absence of SNP correlations to copy number - a feature potentially usable in a SNP-based association study. To arrive at the haplotypes presented in Figure 1-8, I conducted a literature review to assemble known haplotypes and assembled the novel haplotypes using optical mapping. 


\section{Chapter 2. Assembling the alleles of the amylase locus}

\section{Published assemblies}

To assemble the amylase haplotypes I took two broad approaches: re-analyzing previous literature in light of our discoveries and de novo assembly. For the published assemblies, I started with Gumucio et al., who assembled a partial amylase haplotype through restriction mapping ${ }^{163}$. Groot et al. expanded on the work by assembling the first full amylase haplotype with probes ${ }^{145}$. Perry et al. followed, with fiber FISH assemblies of four haplotypes ${ }^{18}$, and Teague et al. used genome-wide optical mapping on three individuals, with the amylase locus uninvestigated ${ }^{164}$. How these haplotypes relate to the ones I found is a story best told through pictures; thus Figures 2-1-4 step through each study's findings and methods ${ }^{9,63-65}$ and how they relate to the findings of this study. Please note that the intergenic region presented in Figure 1-8 is divided into two regions in the graphics below, because it was later found to consist of two segments. 
Key:

\begin{tabular}{|l}
$\square$ AMY2B $\square$ AMY2A $\square A M Y 1$ \\
$\square$ Intergenic A $\square$ Intergenic B $\square$ AMY2A pseudogene -Inverted
\end{tabular}

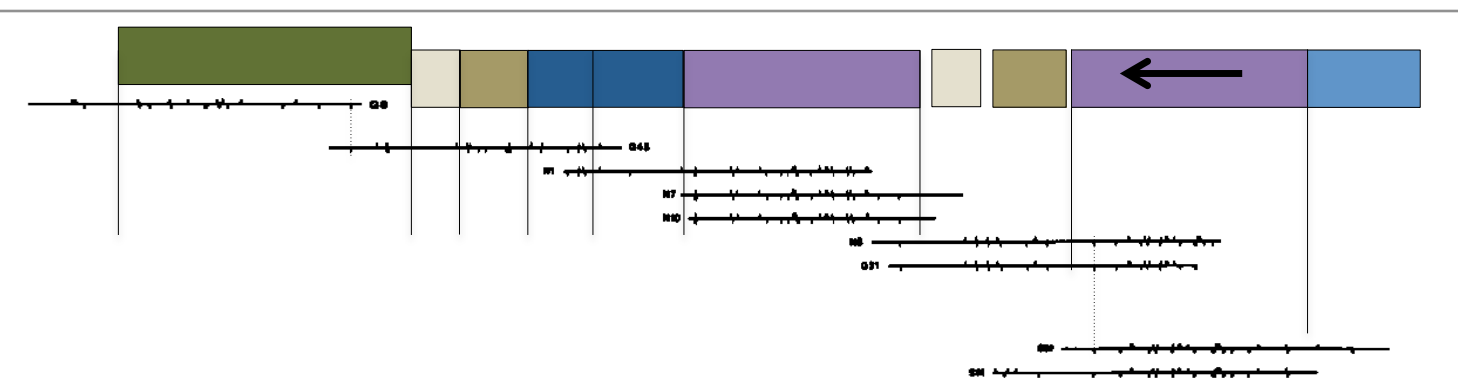

Figure 2-1. Gumucio et al. isolated cosmid clones carrying amylase genes and restriction mapped them using EcoRI and HindIII ${ }^{163}$. The black and white drawing is from the original paper. The horizontal lines are the cosmid inserts (order has been modified). The tick marks on the top and bottom are the EcoRI and HindIII cut sites, respectively. The colored boxes are our defined segments mapping to the assembly.

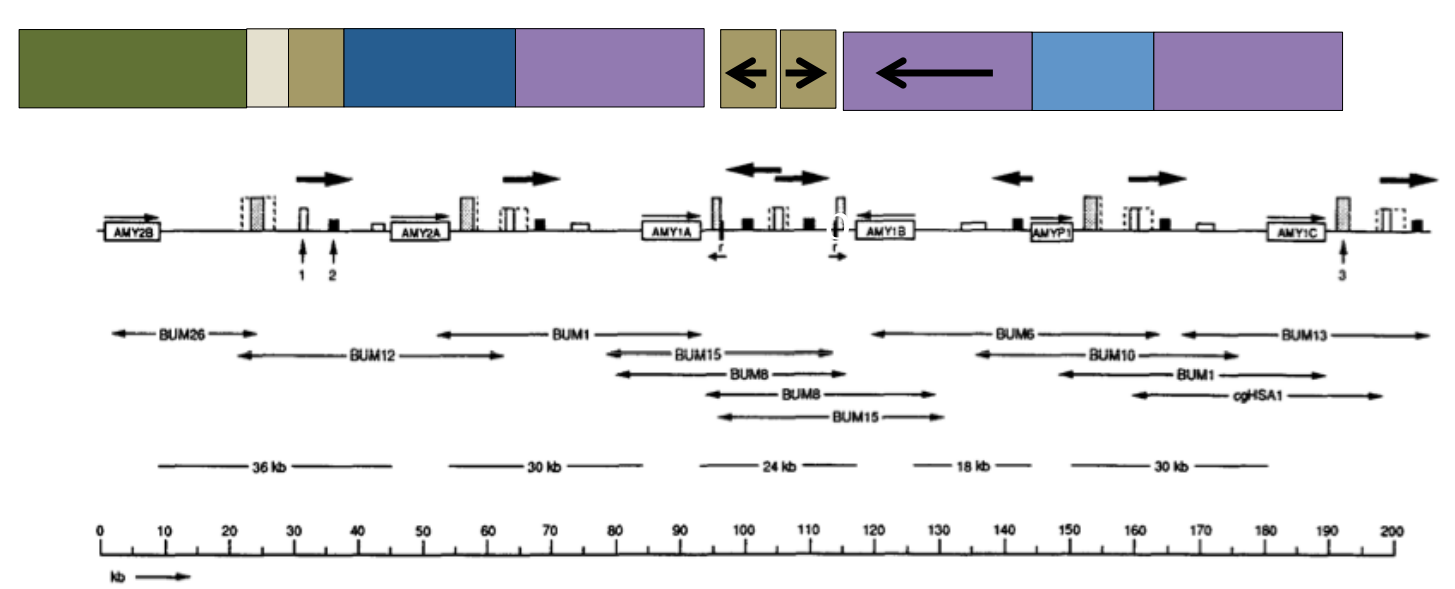

Figure $\overrightarrow{2-2}$. Haplotype AH3 in Groot et al. Cosmids containing amylase genes were isolated and assembled into a contig by mapping the hybridization of restriction fragments ${ }^{145}$. The black and white drawing is the original figure. A size scale is at the bottom and the lines above are the cosmids. The rectangles are the hybridization sites. White $=X b a \mathrm{I}$ fragment. Black $=B g l \mathrm{II}$ fragment. Dotted/Gray $=$ SstI fragment. Above are the modern segments. 
Key:

$\square$ AMY2B $\square$ AMY2A $\square$ AMY1
$\square$ Intergenic $\square$ aMY2A pseudogene $\longleftarrow$ Inverted
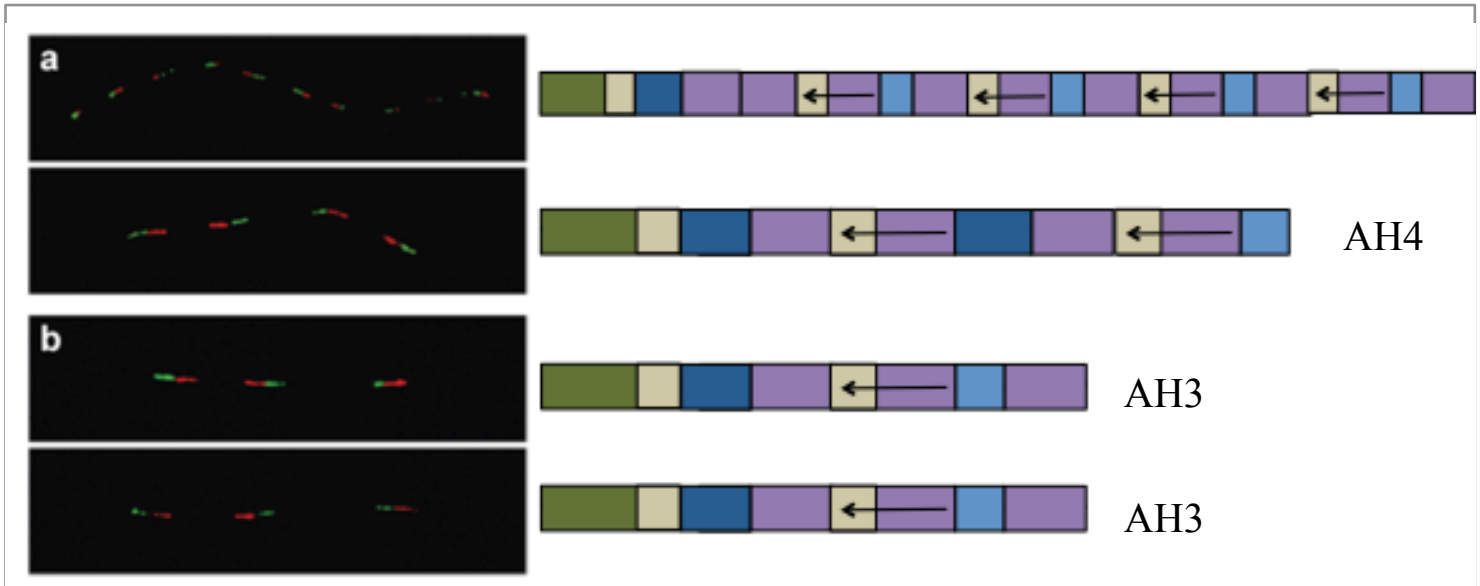

Figure 2-3. Haplotypes AH4 and AH3 in Perry et al. Fiber FISH is a

modification of the FISH technique. DNA fibers are stretched on a glass slide and allowed to hybridize with markers. The resulting fluorescence gives the order of markers on the strand. In the original picture (left), red corresponds to $A M Y 1$ and green to $A M Y 1$ 's promoter ${ }^{18}$. Because this only informs on the location and orientation of $A M Y 1$, the haplotypes to the right have only the inferred locations of $A M Y 2 B$ and $A M Y 2 A$. Of note, we did not find evidence for the upper haplotype in our samples, and the structure we found for the second-most upper haplotype (AH4) did not agree with this assembly. These two haplotypes could potentially be fiber FISH artifacts (personal correspondence with John Armour). 
Key:

$\begin{array}{cl} & \square \text { AMY2B } \square \text { AMY2A } \square A M Y 1 \\ \text { Intergenic A } \square \text { Intergenic B } \square A M Y 2 A \text { pseudogene } \text {-Inverted }\end{array}$

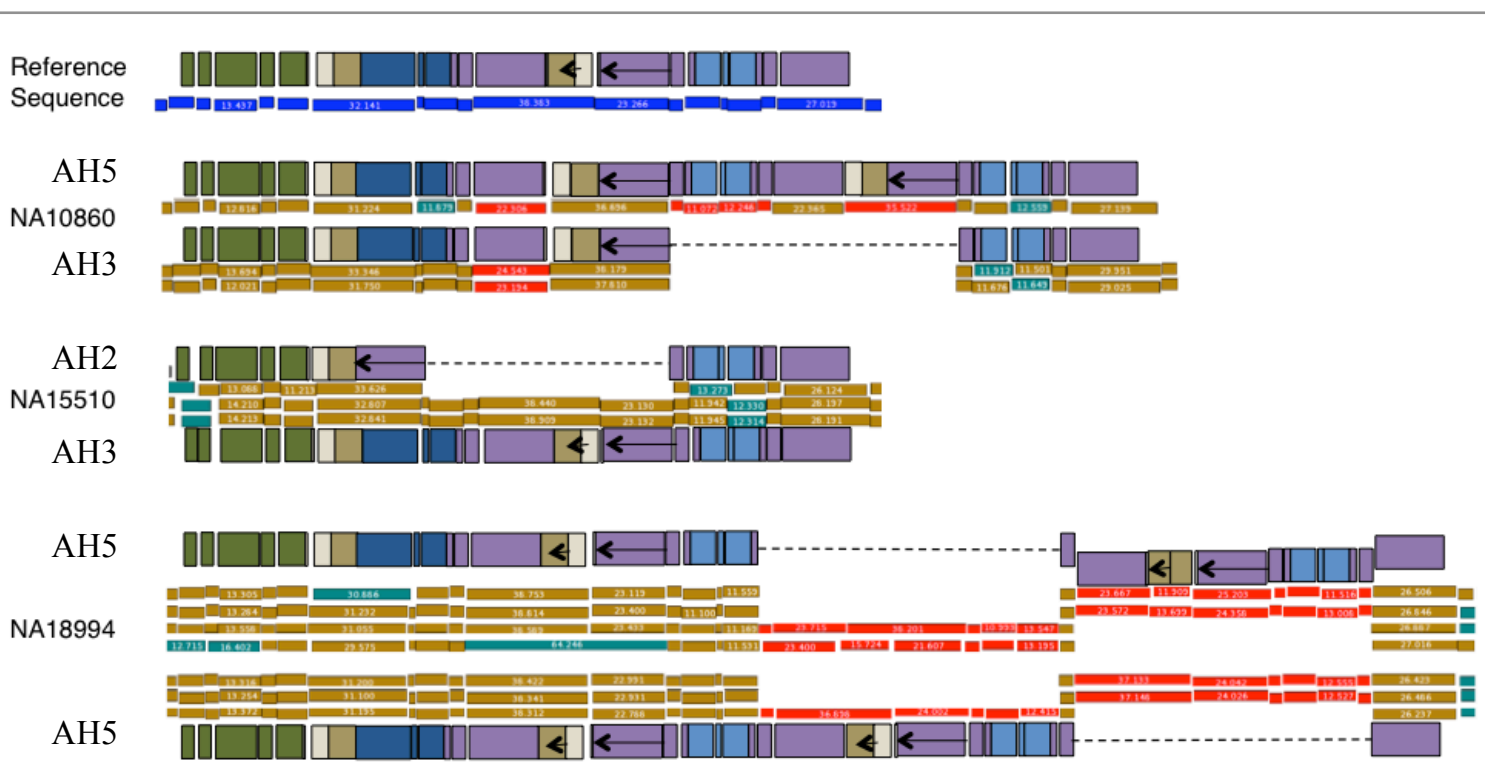

Figure 2-4. Haplotypes AH5, AH3, and AH2 in Teague et al. In this method of optical mapping, DNA is spread on a glass slide and digested with SwaI. The DNA is dyed and imaged, with the cut sites marked by a lack of fluorescence. The gold, red, and teal boxes are screenshots from the companion program that aligned and graphically depicted the data. Each box represents a strand of DNA, with the breaks between boxes representing cut sites. The reference sequence is at the top. Drawn above and below the assemblies are the amylase haplotypes that best match the box/break pattern. Though the existence of AH2 has not been previously reported, it is present in this data ${ }^{164}$. 


\section{Novel assemblies}

After analyzing previously assembled haplotypes, I wanted to assemble the haplotypes that had not yet been captured. To do this, I used optical mapping, the technology of Teague et al., but on the BioNano Genomics platform. The techniques are similar, in that strands of whole DNA are stretched and subjected to a restriction enzyme. However, with the BioNano platform, this enzyme leaves fluorescence at the cut sites (as opposed to the Teague et al. platform, where only the intact DNA is fluorescent). This creates patterns that can be used to assemble a whole genome, or pieces thereof, in a manner similar to restriction mapping. Each amylase gene has its own restriction pattern, and because optical mapping uses whole strands of DNA, I can determine the order and orientation of the genes from these patterns.

I selected two individuals who together had two unreported haplotypes (AH2 and AH2B2), one partially assembled haplotype (AH4), and one known haplotype to serve as a positive control (AH3). The BioNano team did the work of extracting and analyzing the DNA, while I worked with Alex Hastie to align the data (Figure 2-5). The haplotypes that had already been assembled ( $\mathrm{AH} 3$ and $\mathrm{AH} 2)$ were largely consistent. On the other hand, the AH4 structure contradicts the Perry et al. structure (Figure 2-3) with one fewer inverted $A M Y 1$ copies, though Perry et al. could have characterized another variant that was not present in our copy number data. In addition, anonymous samples that BioNano had access to, as well as a European American family, contained AH1, AH3, and AH5, which assembled into structures consistent with the known haplotypes. Of note, in several haplotypes, the $A M Y 2 A$ pseudogene is inverted. This feature appears to be stably inherited, but has not been confirmed using a second technology. 
Figure 2-5. Optical mapping assembly of structural haplotypes. For optical mapping, strands of whole, labeled DNA (nick labeled and backbone stained with YoYo1) DNA are electrophoresed through a NanoChannel array. The array straightens the DNA and the labeling enzyme leaves fluorescence at the nick sites, creating patterns that can be used to assemble a whole genome, or pieces thereof, in a manner similar to restriction mapping. Each amylase gene has its own restriction pattern, so we can determine the order and orientation of the genes from these patterns. 


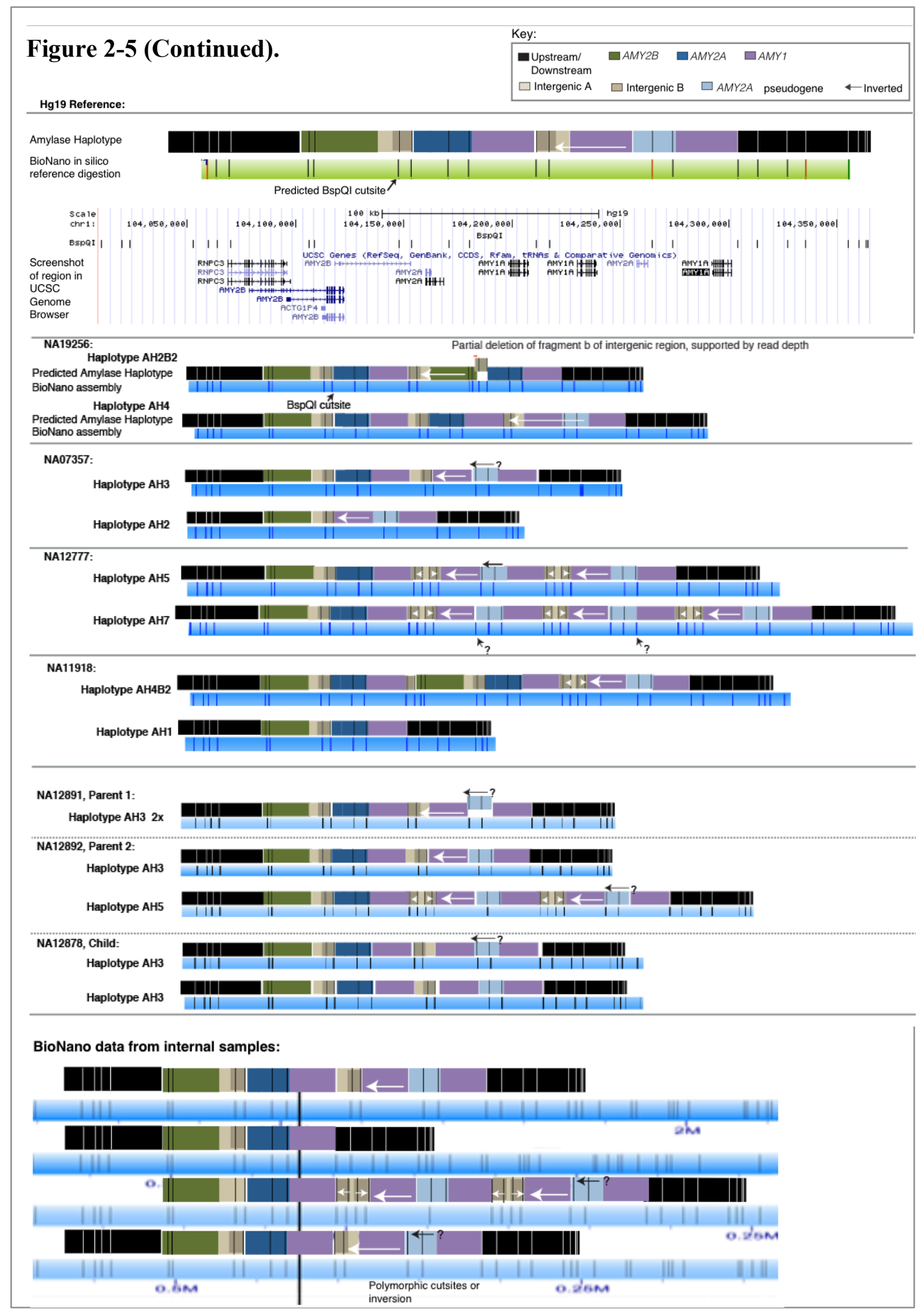




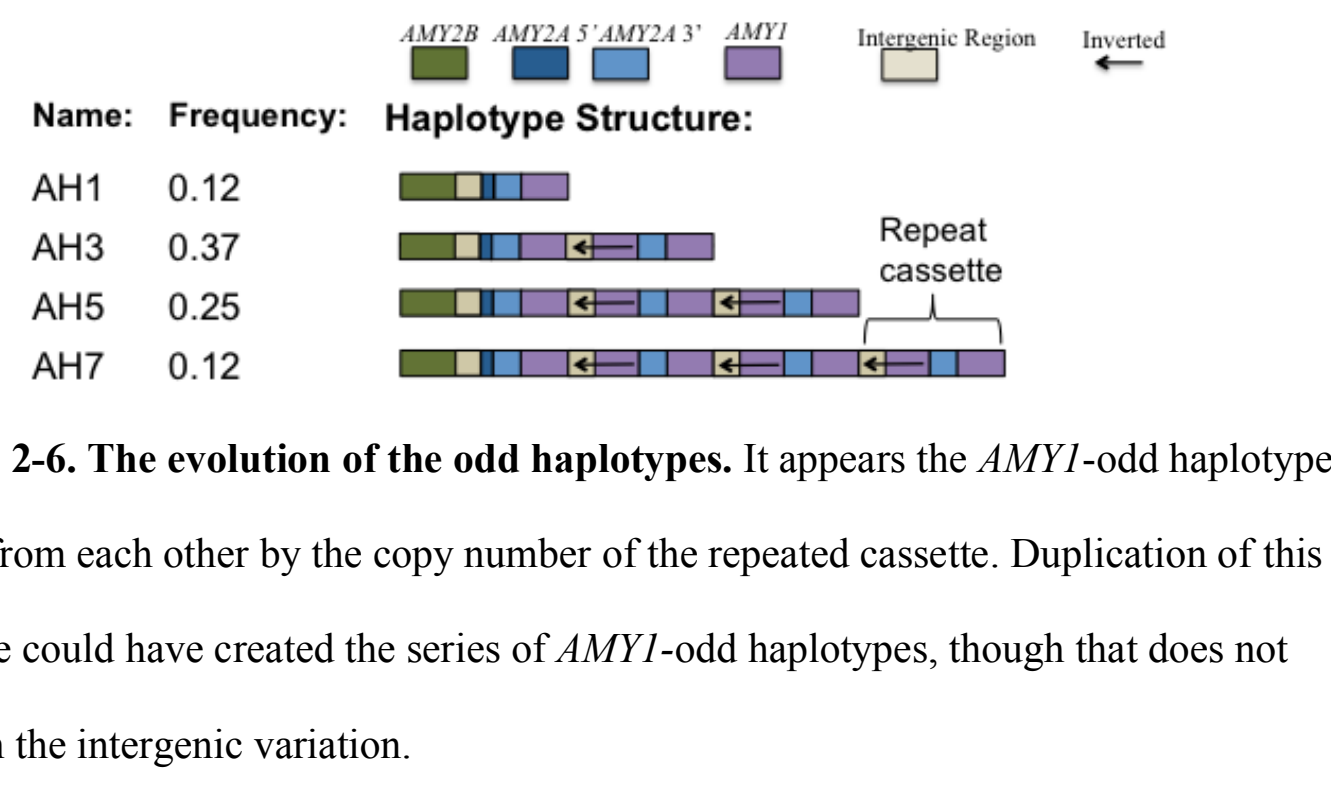

Figure 2-6. The evolution of the odd haplotypes. It appears the $A M Y 1$-odd haplotypes differ from each other by the copy number of the repeated cassette. Duplication of this cassette could have created the series of $A M Y 1$-odd haplotypes, though that does not explain the intergenic variation.
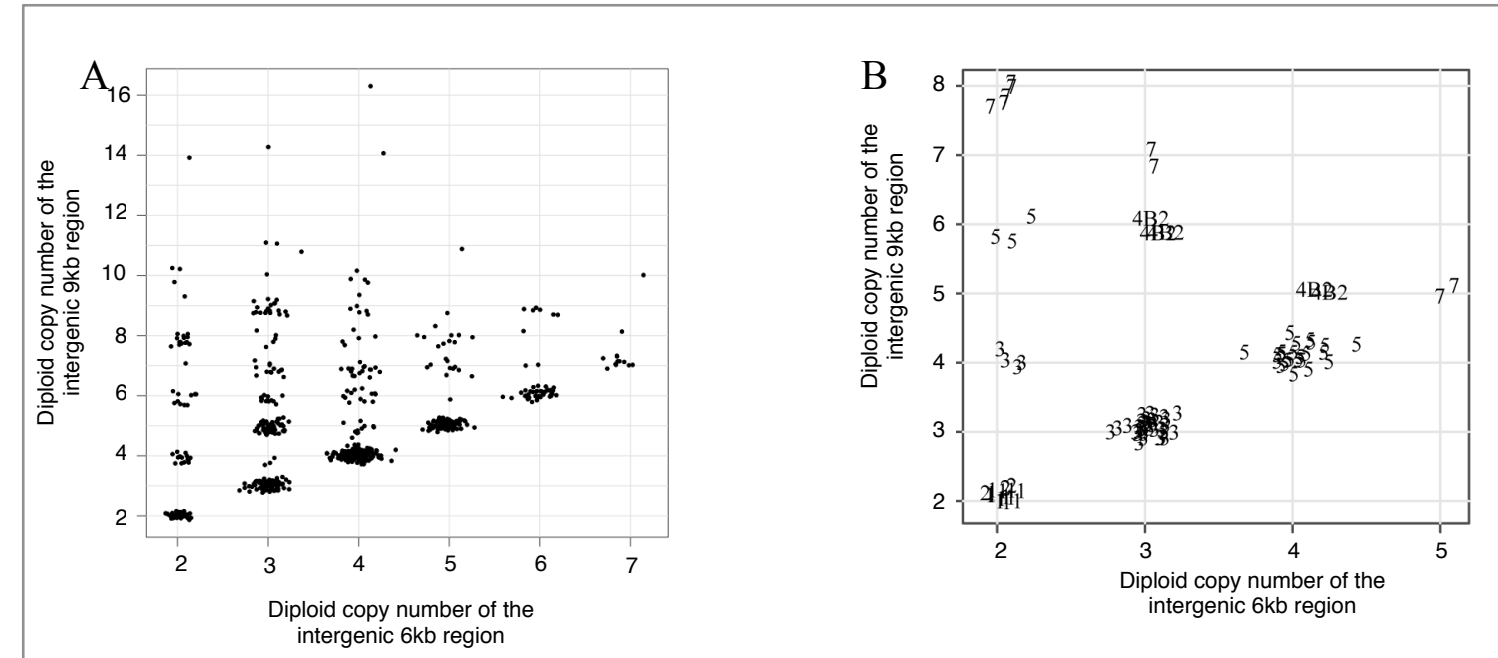

Figure 2-7. The relationship between the two intergenic segments. (A) The diploid copy number of the $9 \mathrm{~kb}$ intergenic segment (Intergenic region $\mathrm{B}$, drawn in other figures as brown) is plotted against the $6 \mathrm{~kb}$ segment (Intergenic region A, drawn in other figures as tan). (B) Individuals with AH2 were selected out of the GPC cohort, and their other haplotype plotted as a number. Numbers off the 1:1 line indicate variation in the intergenic regions of the those haplotypes. Most haplotypes have this variation, with the exceptions of AH1, AH4, AH2, and AH2B2. 
As a set, the structural haplotypes suggest the mutational history of the amylase locus. The more common $A M Y 1$-odd haplotypes (haplotypes containing an odd number of $A M Y 1$ ) differ in the number of copies of a cassette containing two head-to-head $A M Y 1$ genes separated by the last 6 exons of $A M Y 2 A$ (a pseudogene)(Figure 2-6). Frequent nonallelic homologous recombination ${ }^{165}$ (NAHR) involving this tandem array could have generated these many structural forms, but that hypothesis is complicated by the fact that the intergenic region varies, something we would not expect in NAHR ${ }^{165}$. This variation could be coincidental or it could be the scars left behind by other, unknown mutational mechanisms. Either way, with each $A M Y 1$-odd haplotype having at least two different structural forms when accounting for the intergenic region, it suggests these haplotypes each have multiple origination events (Figure 2-7 and -8).

\begin{tabular}{|c|c|c|c|}
\hline Name: & Freq.: & Haplotype Structure: & \\
\hline $\mathrm{AH} 1$ & 0.12 & ш & $\triangle A M Y 2 A$ \\
\hline $\mathrm{AH} 3$ & 0.37 & 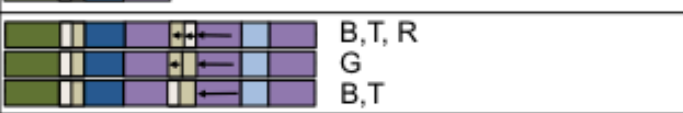 & $\square_{\text {pseudogene }}^{A M Y 2 A}$ \\
\hline $\mathrm{AH} 4$ & 0.01 & 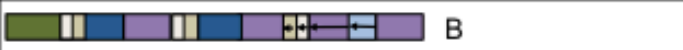 & $L^{A M Y T}$ \\
\hline AH5 & 0.25 & 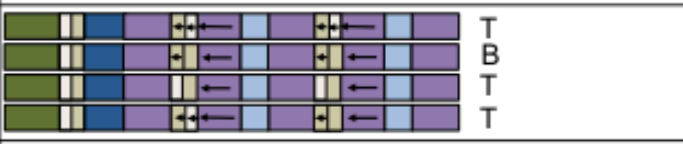 & $\begin{array}{l}\text { Intergenic A } \\
\square \text { Intergenic B } \\
\leftarrow \text { Inverted }\end{array}$ \\
\hline $\mathrm{AH} 7$ & 0.12 & 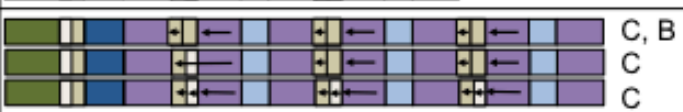 & \\
\hline $\mathrm{AH} 2$ & 0.07 & पझ्षा & \\
\hline AH2B2 & 0.01 & II) $|\mathrm{HF}=1| \mathrm{B}$ & \\
\hline AH4B2 & 0.05 & 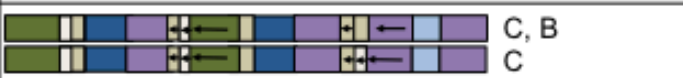 & \\
\hline
\end{tabular}

Figure 2-8. A list of haplotypes, including the intergenic variation. $G$ = haplotype assembled by Groot et al.; T=Teague et al. optical mapping; B=BioNano optical mapping; $\mathrm{C}=$ inferred from copy numbers; $\mathrm{R}=$ reference haplotype. 
As opposed to the $A M Y 1$-odd haplotypes, the $A M Y 1$-even haplotypes do not appear to have a mutational history in common. The lack of commonalities in this group suggests these haplotypes most likely have separate origination events, one each. A possible exception is AH4B2, which has variation in its intergenic region, though it is unclear whether that indicates two origins or a mutation after the origin.

Given that the more common $A M Y 1$-odd haplotypes originated multiple times, it was unlikely there were any SNPs that would correlate well with haplotype or with copy number. Polymorphisms within the actual, varying part of the amylase locus proved hard to genotype, and with the multiple structural forms of the amylase locus, surrounding SNPs likely had low correlations with copy number. However, even low correlations can be useful given a large enough sample size. Because of this, I went on to investigate and quantify the relationships the surrounding SNPs have to the amylase locus. 


\section{Chapter 3. SNP correlations to the amylase locus}

I first visualized the relationships SNPs had with the amylase locus by making a spiderplot using the individuals I could phase $(\mathrm{n}=158)($ Figure 3-1). In this plot, the SNP haplotypes on either side of the locus are clustered as a phylogenetic tree, with the amylase structural haplotypes on them plotted at the distal ends.

AH2 clustered on one branch, as did AH2B2 and AH4B2, in both the GPC cohort and 1000 Genomes, suggesting that the structural data are correct: each of these haplotypes has one origin, with the possible exception of AH4B2. Haplotype AH4 did not cluster consistently between cohorts, though it is a low frequency haplotype with less than three instances per cohort.

Unlike the $A M Y 1$-even haplotypes, the more common $A M Y 1$-odd haplotypes are intermixed on nearly every branch, indicating that most have arisen at least 7 times (one for each SNP haplotype branch). It is possible that each branch has its own set of haplotype variants, as marked by the intergenic region; however, I did not have a large enough cohort to investigate these sub-groups of haplotypes. Such an endeavor would likely require hundreds of parent-child trios to obtain enough phase-able individuals and a method that can genotype which intergenic regions have which structure. 


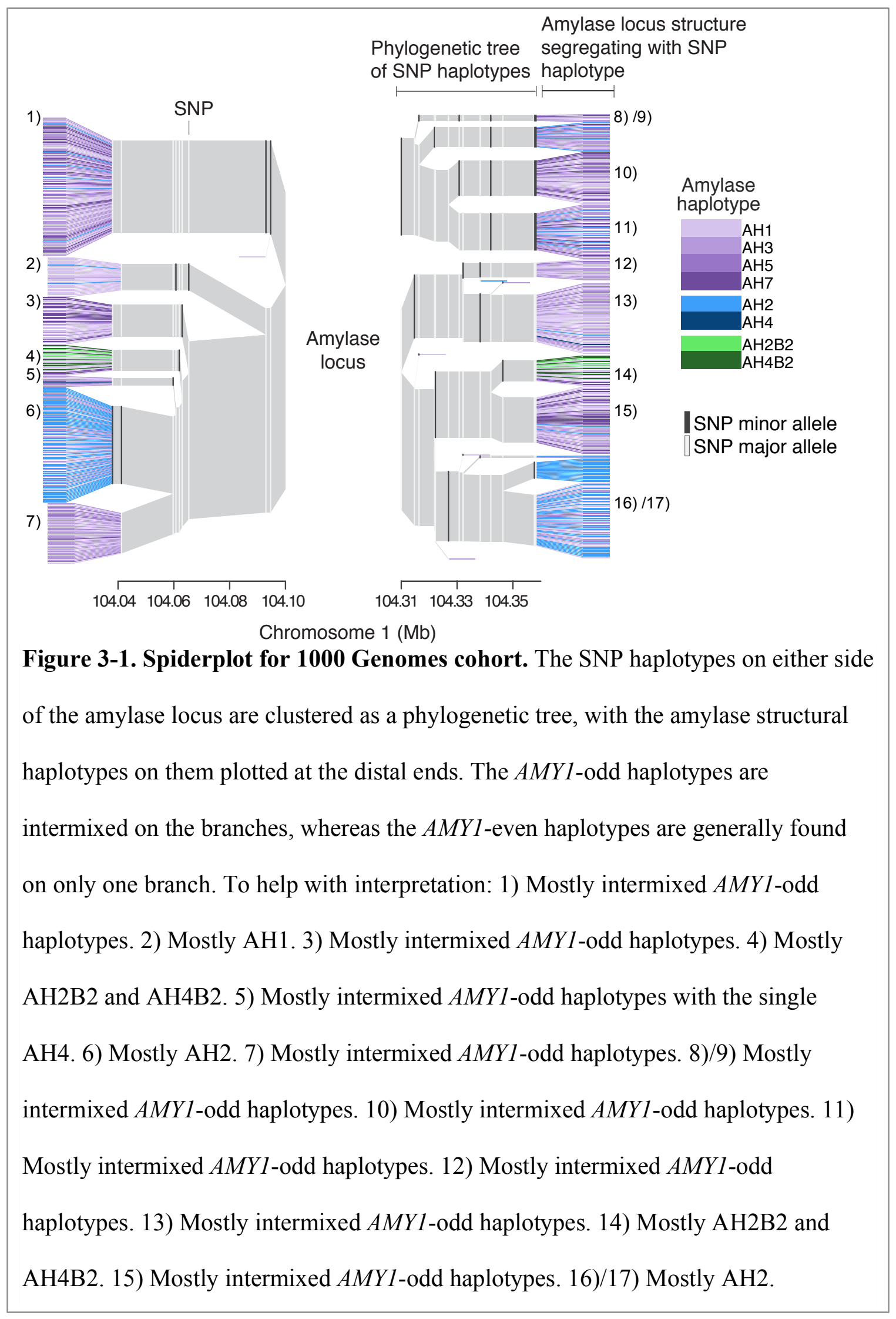




\section{SNPs capturing structural haplotypes}

Table 3-1. The analysis displayed was conducted in the GPC cohort, due to better SNP coverage and genotyping.

\begin{tabular}{cccccccccc}
\hline $\begin{array}{c}\text { Haplotype } \\
\text { (AH\#) }\end{array}$ & $\begin{array}{c}\text { Associated } \\
\text { SNP }\end{array}$ & $\begin{array}{c}\text { Minor } \\
\text { Freq. }\end{array}$ & $\begin{array}{c}\mathrm{r}^{2} \\
\text { of }\end{array}$ & \multicolumn{2}{c}{$\begin{array}{c}\text { Replicated } \\
\text { s- }\end{array}$} & $\begin{array}{c}\mathrm{r}^{2} \\
\text { in 1000 }\end{array}$ & $\begin{array}{c}\text { value } \\
\text { of }\end{array}$ & $\begin{array}{c}\text { Replicated } \\
\text { in } 1000\end{array}$ \\
\hline 1 & rs113922683 & 0.19 & 0.22 & $<10^{-6}$ & yes & 0.23 & $<10^{-6}$ & yes \\
3 & rs34848656 & 0.26 & 0.08 & $<10^{-6}$ & no & 0.23 & $<10^{-6}$ & yes \\
5 & rs1566154 & 0.24 & 0.07 & $<10^{-6}$ & no & 0.03 & $<10^{-3}$ & no \\
7 & s75133138 & 0.16 & 0.19 & $<10^{-6}$ & yes & 0.09 & $<10^{-5}$ & yes \\
2 & rs72694406 & 0.43 & 0.32 & $<10^{-6}$ & yes & 0.43 & $<10^{-6}$ & yes \\
4 & rs12740780 & 0.12 & 0.26 & $<10^{-6}$ & no & 0.37 & $<10^{-6}$ & no \\
2B2 & rs12076610 & 0.18 & 0.11 & $<10^{-6}$ & yes & 0.44 & $<10^{-6}$ & no \\
4B2 & rs79043596 & 0.17 & 0.27 & $<10^{-6}$ & yes & 0.24 & $<10^{-6}$ & no \\
\hline
\end{tabular}

Table 3-2. The "BMI association $P$ value" refers to data from the GIANT consortium, discussed in Chapter 4.

\begin{tabular}{|c|c|c|c|c|c|c|c|c|c|}
\hline \multirow[b]{3}{*}{ Gene } & \multirow[b]{3}{*}{ SNP } & \multirow{3}{*}{$\begin{array}{c}\text { Minor } \\
\text { Allele } \\
\text { Freq. }\end{array}$} & \multicolumn{6}{|c|}{$A M Y I$ copy number association } & \multirow{3}{*}{$\begin{array}{c}\text { BMI } \\
\text { association } \\
P \text { value }\end{array}$} \\
\hline & & & $\begin{array}{r}\text { Chan } \\
\text { number }\end{array}$ & $\begin{array}{l}\text { n copy } \\
\text { nor allele }\end{array}$ & \multicolumn{2}{|c|}{$r^{2}$} & \multicolumn{2}{|c|}{$P$ value } & \\
\hline & & & GPC & GoT2D & GPC & GoT2D & GPC & GoT2D & \\
\hline \multirow[t]{10}{*}{$A M Y I$} & rs4244372 & 0.33 & -1.23 & -1.25 & 0.111 & 0.118 & $<10^{-6}$ & $<10^{-6}$ & 0.09 \\
\hline & rs 11577390 & 0.07 & 2.08 & 1.88 & 0.104 & 0.089 & $<10^{-6}$ & $<10^{-6}$ & 0.13 \\
\hline & rs 1566154 & 0.19 & 0.90 & 0.88 & 0.044 & 0.038 & $<10^{-6}$ & $<10^{-6}$ & 0.11 \\
\hline & rs 1930212 & 0.18 & -0.89 & -1.05 & 0.041 & 0.053 & $<10^{-6}$ & $<10^{-6}$ & 0.74 \\
\hline & rs 10881197 & 0.35 & -0.66 & -0.73 & 0.037 & 0.042 & $<10^{-6}$ & $<10^{-6}$ & 0.75 \\
\hline & rs 2132957 & 0.03 & -1.95 & -1.29 & 0.036 & 0.022 & $<10^{-6}$ & $<10^{-6}$ & 0.73 \\
\hline & rs 11185098 & 0.26 & 0.70 & 0.79 & 0.032 & 0.035 & $<10^{-6}$ & $<10^{-6}$ & 0.80 \\
\hline & rs 1999478 & 0.18 & -0.76 & -0.92 & 0.030 & 0.042 & $<10^{-5}$ & $<10^{-6}$ & 0.53 \\
\hline & rs 1330403 & 0.14 & 0.82 & 0.75 & 0.029 & 0.020 & $<10^{-6}$ & $<10^{-6}$ & 0.42 \\
\hline & rs 6696797 & 0.35 & -0.60 & -0.72 & 0.028 & 0.041 & $<10^{-5}$ & $<10^{-6}$ & 0.63 \\
\hline$A M Y 2 B$ & rs 12076610 & 0.11 & 0.80 & 0.61 & 0.582 & 0.479 & $<10^{-6}$ & $<10^{-6}$ & ND \\
\hline$A M Y 2 B$ & rs 11185098 & 0.26 & 0.35 & 0.24 & 0.207 & 0.166 & $<10^{-6}$ & $<10^{-6}$ & 0.80 \\
\hline$A M Y 2 A$ & rs 28558115 & 0.11 & 0.90 & 0.72 & 0.398 & 0.270 & $<10^{-6}$ & $<10^{-6}$ & ND \\
\hline$A M Y 2 A$ & rs 11185098 & 0.26 & 0.42 & 0.32 & 0.154 & 0.112 & $<10^{-6}$ & $<10^{-6}$ & 0.80 \\
\hline
\end{tabular}

Confirming the patterns of clustering in the spiderplot, I did not find a SNP that tagged any structural haplotype with an $r^{2}$ greater than 0.32 , and imputation faired only slightly better (imputation is a process that involves correlating whole SNP haplotypes with the structural haplotypes) (Table 3-1). In both methods, AH2 was the best correlated, with an $\mathrm{r}^{2}$ of 0.32 for a tag SNP (rs72694406) and 0.43 for imputation. With 
its spiderplot clustering, one would expect the correlations for $\mathrm{AH} 2$ to be higher, but there were other amylase haplotypes on its branch and $\mathrm{AH} 2$ appeared on other branches at a low frequency, possibly indicating errors in genotyping or phasing.

\section{SNPs capturing copy number}

Even if SNPs do not have good correlations $\left(r^{2}>0.8\right)$ to amylase structural haplotypes, they can still have a correlation with gene copy number, a measurement that is used in association studies. I correlated the AMY1 copy number of the Europeanancestry individuals of the 1000 Genomes Project ${ }^{157}$ to their SNP genotypes and found SNPs that had an average difference of 0.6 to $2.0 A M Y 1$ copies per SNP minor allele. Because of the non-normal distribution of amylase copy numbers, the $P$ value of the correlations had to be determined by permutation

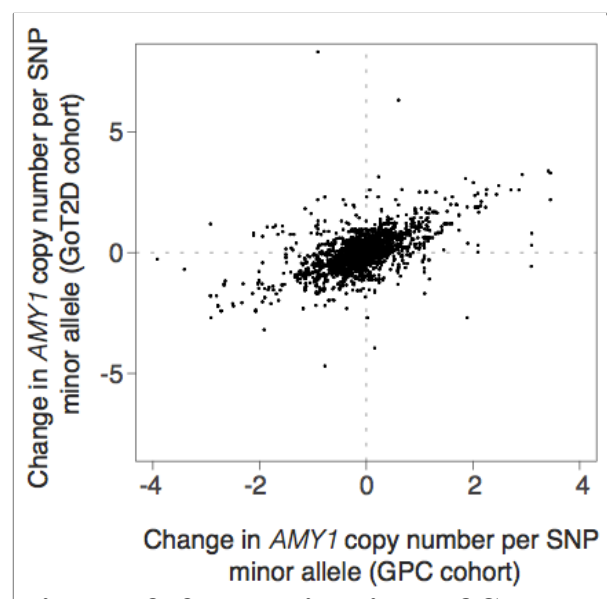

Figure 3-2. Replication of SNP correlations. SNP correlations to AMY1 copy number were compared across cohorts, revealing that the effects generally replicate.

tests. The amylase copy numbers were shuffled and a new set of correlations were calculated one million times; the $P$ value was the fraction of permutations that produced a better result than the observed result. These tests established that the correlations were statistically significant. These effect sizes and $r^{2}$ values replicated in two independent cohorts: 768 and 2,807 European-ancestry individuals in the GPC and Got2D cohorts, respectively (Figure 3-2 and Table 3-2). This shows that, despite poor correlations, the SNPs do contain information on the amylase locus. 


\section{Chapter 4. Associating the amylase locus to obesity}

After I (with the help of Bob Handsaker and Alex Hastie) finished characterizing the locus, it was time to test it for associations to phenotypes. As chance would have it, during our investigation into the amylase locus, Falchi et al. reported that each copy of $A M Y 1$ decreased the risk of obesity 1.2 fold $^{28}$, a profound effect considering $A M Y 1$ copy number varies so widely. This effect would explain $11 \%$ of the genetic contribution to obesity, far greater than the effect of fat mass and obesity-associated protein $(F T O)$ - the largest effect detected thus far - yet amylase went undetected in other association studies, particularly the GIANT Consortium's GWAS of 339,224 people for body mass index $(\mathrm{BMI})^{28,94,166}$. This finding provided a good opportunity to accomplish the goal of my dissertation; I could demonstrate how precise genotypes, knowledge of the locus, and utilizing the surrounding SNPs could lead to a well-supported, and potentially replicable, result that would confirm or contradict the previous study ${ }^{28}$.

I first used the surrounding SNPs to interrogate the locus. Although the SNPs capture only a small fraction of $A M Y 1$ copy number variation, statistical power is the product of linkage disequilibrium $\left(r^{2}\right)$ and sample size. Given the GIANT Consortium's large cohort of 339,224 individuals ${ }^{94,166}$ and the reported $A M Y 1$ copy number effect on 
$\mathrm{BMI}^{28}$, the SNPs most correlated with amylase would be $99 \%$ likely to be nominally associated $(P<0.05$, calculated by Tõnu Esko) in the GIANT GWAS. However, none of the 17 SNPs covered by GIANT reached even nominal significance, and the SNPs as a group showed no trend toward low association statistics (Table 3-1 and Figure 4-1).

Since this lack of evidence for $A M Y l$ 's association to $\mathrm{BMI}$ is indirect, we conducted our own association analyses by directly measuring the copy numbers of the amylase genes using our high-resolution methods in three different cohorts of Europeans. Tõnu Esko did the majority of the statistics, Robert Handsaker ran Genome STRiP, Jennifer Moon did the SNP genotyping, and I led the project.

We began by analyzing 1,000

Estonians selected from a broader Estonian Biobank cohort ${ }^{167}$ (51,535 individuals) for 
being in the tails of the BMI distribution -500 individuals with $\mathrm{BMI}<22$ and 500 individuals with BMI $>33$, making a lean/obese case-control cohort. The individuals were randomized onto 96-well plates, and we were blinded to their case/control status. We observed an association to the SNPs that were associated with BMI in earlier studies ${ }^{94}$, including SNPs at the fat mass and obesity-associated protein $\left(F T O, P=3.5 \times 10^{-7}\right)$, SEC16 homolog B $\left(S E C 16 B, P=5.3 \times 10^{-4}\right)$, and mitochondrial carrier homolog 2 $\left(\mathrm{MTCH} 2, P=9.6 \times 10^{-3}\right)$ loci. For added power to detect a positive-control association, we also tested a group of 11 known obesity/BMI SNPs, referred to collectively as the polygenic score, and found the score was associated $\left(P=3.7 \times 10^{-12}\right)$ (Table 4-1,

\section{Appendix B: Table B-2).}

With these positive controls validating the study design and demonstrating power to detect the effect of $A M Y 1$, I used ddPCR to obtain crisp, integer genotypes of all three amylase genes, again observing the abundance of even $A M Y 1$ copy numbers. With our study design, we had $>99 \%$ power to detect (at nominal significance) effects as strong as those reported ${ }^{28}$. However, we did not observe any association between obesity and the copy number of any amylase gene ( $P=0.70$ for $A M Y 1)$ (Figure 4-2).

We then analyzed two other cohorts of Europeans - one consisting of 2,807 individuals $(1,437$ type 2 diabetes cases and 1,370 controls) sequenced to $>5 \mathrm{x}$ average coverage (GoT2D cohort), and the other of 657 Europeans sequenced to 7x median coverage (InCHIANTI cohort ${ }^{168}$, completed with the help of Michael Weedon and Marcus Tuke). Analysis of amylase gene copy number using Genome STRiP again showed the enrichment of even copy numbers, replicating our earlier results and validating the precision of the analysis within the new cohorts. The GoT2D cases and 
controls each had $95 \%$ power, while InCHIANTI had $77 \%$ power, to detect the reported effect $^{28}$ of $A M Y 1$ at nominal significance. Yet $A M Y 1$ copy number did not associate with BMI in any group $(P=0.31$ for GoT2D controls, $P=0.24$ for GoT2D cases, $P=0.53$ for InCHIANTI), or in a meta-analysis of all 3,464 replication samples $(P=0.38)$. By contrast, SNPs at FTO and other loci showed the associations expected given sample size and statistical power.

Table 4-1. The association results of the three cohorts.

\begin{tabular}{|c|c|c|c|c|c|c|}
\hline Cohort & $\begin{array}{c}\text { Sample } \\
\text { size }\end{array}$ & Gene & $\begin{array}{l}\text { Variant } \\
\text { genotyped }\end{array}$ & Power & Odds ratio, obesity & $P$ value \\
\hline \multirow[t]{4}{*}{ Estonian } & \multirow[t]{4}{*}{1000} & FTO & rs 1558902 & 0.96 & $1.61(1.34-1.93)$ & $3.5 \times 10^{-7}$ \\
\hline & & Polygenic & $11 \mathrm{SNPs}$ & $>0.99$ & $1.61(1.41-1.84)$ & $3.7 \times 10^{-12}$ \\
\hline & & $A M Y 1$ & Copy number & $>0.99$ & $1.01(0.96-1.06)$ & 0.7 \\
\hline & & & & & Beta, BMI & \\
\hline \multirow[t]{3}{*}{ InCHIANTI } & \multirow[t]{3}{*}{657} & FTO & rs1558902 & 0.32 & $0.41(-0.04-0.86)$ & 0.07 \\
\hline & & Polygenic & 11 SNPs & 0.64 & $0.50(0.20-0.81)$ & 0.001 \\
\hline & & $A M Y 1$ & Copy number & 0.77 & $0.04(-0.08-0.15)$ & 0.53 \\
\hline \multirow{3}{*}{$\begin{array}{l}\text { GoT2D } \\
\text { controls }\end{array}$} & \multirow[t]{3}{*}{1370} & FTO & rs 1558902 & 0.58 & $0.03(-0.02-0.07)$ & 0.22 \\
\hline & & Polygenic & $11 \mathrm{SNPs}$ & 0.91 & $0.08(0.04-0.12)$ & $4.2 \times 10^{-4}$ \\
\hline & & $A M Y 1$ & Copy number & 0.95 & $0.01(-0.01-0.03)$ & 0.31 \\
\hline \multirow{3}{*}{$\begin{array}{c}\text { GoT2D } \\
\text { cases }\end{array}$} & \multirow[t]{3}{*}{1437} & FTO & rs1558902 & 0.60 & $0.03(-0.01-0.07)$ & 0.21 \\
\hline & & Polygenic & $11 \mathrm{SNPs}$ & 0.93 & $0.06(0.02-0.10)$ & $6.3 \times 10^{-3}$ \\
\hline & & $A M Y 1$ & Copy number & 0.95 & $0.01(-0.01-0.03)$ & 0.24 \\
\hline \multirow{3}{*}{$\begin{array}{c}\text { GoT2D } \\
\text { meta-analysis }\end{array}$} & \multirow[t]{3}{*}{2807} & FTO & rs1558902 & 0.87 & $0.05(0.02-0.08)$ & $4.2 \times 10^{-3}$ \\
\hline & & Polygenic & 11 SNPs & $>0.99$ & $0.10(0.06-0.13)$ & $3.9 \times 10^{-8}$ \\
\hline & & $A M Y 1$ & Copy number & $>0.99$ & $0.01(-0.01-0.02)$ & 0.44 \\
\hline
\end{tabular}



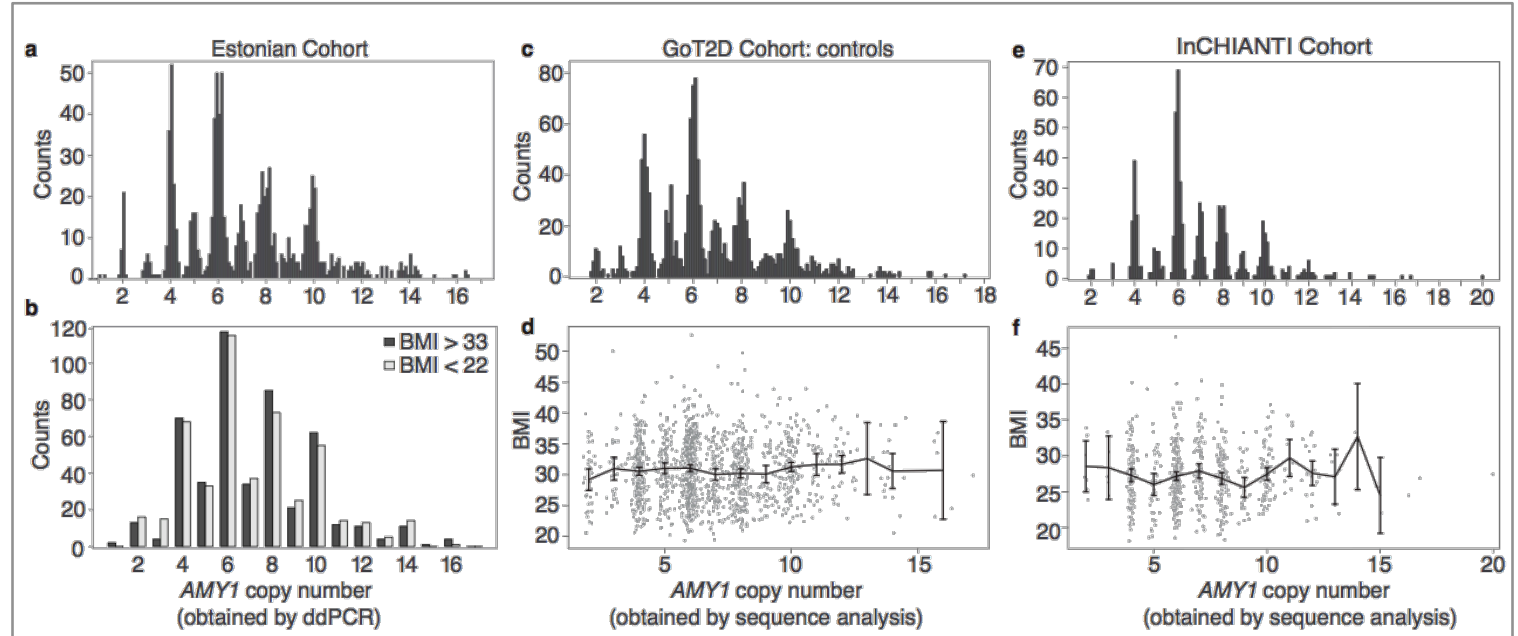

Figure 4-2. The association analysis of $A M Y 1$ copy number to obesity or BMI in

three cohorts. (a) Measurements of $A M Y 1$ copy number in the Estonian cohort. (b)

Obese and lean individuals show indistinguishable distributions of $A M Y 1$ copy

number $(P>0.05)$. (c) Measurements of $A M Y 1$ copy number and (d) association are shown for the GoT2D cohort controls. (e) Measurements of $A M Y 1$ copy number and (f) association are shown for the InCHIANTI cohort.

These results contrast with those of Falchi et al., who found that AMY1 copy number influenced obesity with an odds ratio of 1.19 per copy and changed BMI by -0.15 per copy, both with a $P$ value on the order of $10^{-10}$. Though I have not found a definitive explanation for this contradiction (discussed in the Discussion), I believe it likely stems from my use of higher-resolution genotyping approaches. Though low-resolution calls cannot cause a spurious association, they may conceal confounds that can.

Though we cannot specify a reason why our results contradict, I did accomplish what I set out to do: propose and utilize a study design that is resistant to the known confounds and can replicate across cohorts and genotyping methods. 


\section{Discussion}

\section{Discussion of the previous report}

My results contrast with those of Falchi et al., who found that $A M Y 1$ copy number influenced obesity with an odds ratio of 1.19 per copy and changed BMI by -0.15 per copy, both with a $P$ value on the order of $10^{-10}$. Though I have not found a definitive explanation for this contradiction, I believe it likely stems from my use of higherresolution genotyping approaches.

The previous study failed to see an enrichment in even $A M Y 1$ copy numbers in any of their reported genotyping runs (6868 samples with qPCR). The qPCR assay was purchased from Life Technologies, mapping with undisclosed coordinates to somewhere in exon 1 , which is $98 \%$ identical to the exon 1 of $A M Y 2 A$ and $B$. The copy numbers obtained from this assay do not appear to cluster near integers, based on the raw data in their Figure 3a,b (presented as $\Delta \Delta \mathrm{Ct}$, copy number $\left.=6 /\left(2^{\wedge} \Delta \Delta \mathrm{Ct}\right)\right)$. Despite their assay potentially binding the other amylase genes, their $A M Y 1$ copy number distribution appeared to be shifted left from my distribution. To make my distribution match theirs, I had to multiply my calls by a random number between 0.5 and 1.1 , suggesting that their assay was noisier than mine and had a tendency to underestimate copy number. 
The copy numbers from their replication runs with ddPCR and read-depth (the algorithm mrCaNaVar) actually clustered near integers and had an enrichment in particular copy numbers; however, only a few samples were analyzed with those alternative methods ( 32 for read depth and 96 for ddPCR) and no association analyses were conducted using those calls. Two other methods were used for association analysis: an RNA expression microarray using adipose tissue and expression assays using serum. I was unable to find the probe data or the genomic coordinates of the microarray probes (proprietary property of Affymetrix), so I cannot comment on them. However, whether $A M Y 1$ is expressed in adipose appears to be controversial, with some studies finding it $^{169,170}$ and others not ${ }^{110}$. The expression assay using serum was based on enzymatic activity and utilized a product that had, afterwards, been recalled and discontinued by Roche for producing falsely low results in particular samples.

Though the low-resolution qPCR calls cannot cause a spurious association, they may conceal confounds that can. However, it is difficult for me to pinpoint a single confound that could cause an association in all the cohorts of Falchi et al., because they had multiple cohorts drawn from populations and case/control studies. If it is indeed a spurious association, it may be caused by a constellation of small, hard-to-identify artifacts, perhaps some cohort-specific. I sought to determine how the cohorts were collected, how the DNA was collected, and whether there were different ancestries in the cohort (Falchi et al. stated they were all Europeans, with the exception of the Singapore cohort, and so did not appear to correct for cryptic ancestry differences). Unfortunately, there was not much information available on some of these cohorts. What I found is assembled below: 
- TwinsUK. Used by Falchi et al. as a population cohort and as case/control cohort (using the tails of the BMI distribution). It is from the St. Thomas' UK adult twin registry, part of the Wellcome Trust ${ }^{171}$. This cohort has both DNA from blood and lymphoblastoid cell lines, and I cannot determine which DNA was used. This cohort also appears to contain non-European ancestry individuals, though presumably Falchi et al. screened those out.

- DESIR. Used by Falchi et al. as a population cohort and as a case/control cohort (using the tails of the distribution). It is from The DESIR Study Group ${ }^{172}$ : French individuals in a longitudinal study on Diabetes. Falchi et al. used the controls and freshly extracted the DNA, but didn't state whether it was from blood or tissue and I cannot determine which source DESIR normally collects. This group is composed of French citizens, so may contain non-European individuals.

- AOB. Used as a case/control cohort. This cohort is from the Pasteur Institute. Falchi et al. refers to them as French, but the original study states they had collected different subsets of European ${ }^{173}$. The original study did not state what they did to avoid batch effects in DNA extraction, and their cases were defined as either having morbid obesity or early onset obesity (before 6 years of age). Falchi et al. used what appears to be an undefined subset of 563 samples (out of 2796).

- SP2. Used as a case/control cohort. It is a Singaporean-based cohort with a population mix of Chinese, Malays, and Asian-Indians ${ }^{174}$. Though it appears to be used in many studies, I cannot find information on the methods of collection. It appears to have originally been a population sample, so presumably Falchi et al used the tails of the BMI distribution to assemble a case/control cohort. 
- ABOS. Used in the serum expression assays. This cohort is from Lille University Hospital, France. It does not specify a population on their website (https://clinicaltrials.gov/ct2/show/NCT01129297), but Falchi et al. refers to them as French samples.

- Swedish Families. Used as a case/control cohort in the RNA expression assay. I believe the official name is the SOS Sib Pair study ${ }^{175}$, in which siblings discordant for obesity were collected along with their families. The original study analyzed gene expression in adipose tissue, and Falchi et al. appears to have re-used that data. I cannot find any information on what the cohort collectors did to prevent batch effects.

We also considered the possibility that our study could have failed to see a real effect. We used an Estonian cohort, which is a population with a slightly different diet than that of other European countries ${ }^{176}$, though it appears to be similarly rich in starch ${ }^{177}$. In addition, our two European cohorts had an elevated average BMI, and though it is possible that amylase acts in ways that are specific to lean individuals, we saw no evidence for this in our BMI cohorts (Appendix B, Table B-3) and note that other BMIassociated variants have tended to associate in ways that are consistent across the BMI spectrum $^{178}$.

\section{Discussion of my proposed study model}

Fully understanding human genetic variation and its relationship to phenotypes will require characterizing hundreds of complex loci that mutate at high frequencies, causing large-scale changes to gene dosage and confounding older methods of 
genotyping. The amylase locus offers several insights to guide future studies of these loci:

1) The copy numbers found at any particular locus most likely arise combinatorially from a modest number of structural alleles, and understanding these alleles may assist association studies. In the case of amylase, the knowledge that $A M Y 1$ and $A M Y 2 A$ share parity, confirmed by the structural haplotypes, led to better genotypes (Chapter 1).

2) Structurally complex loci may have both common and rare variation within them, reflecting both ancient and recent mutations, and may be best understood through combinations of analysis methods developed for each, including tagging, imputation, and direct measurement. In the case of amylase, direct measurement was ideal, but if the duplication of $A M Y 2 B$ was of scientific interest, the use of tagging or imputation may have been possible due to its evolutionary history.

3) Although GWAS may miss or greatly under-estimate the relationships of structurally complex loci to phenotypes, accurately-typed SNP markers are useful for understanding and constraining expectations about the strength of an mCNV's potential effect on phenotypes.

Each of these points relies on having accurate measurements of copy number, which can be difficult when using the older genotyping methods ${ }^{48-50,89,90}$. Several emerging technologies have promise for acquiring precise copy number measurements that cluster at integers. The paralog ratio test (PRT, a technique that compares the amount of amplification product between a control and target locus that both use the same primers) has been used effectively in mCNV associations, often giving copy number 
measurements with enough resolution to detect batch effects ${ }^{50,89}$, and ddPCR, as shown here, may also be used. However, PRT and ddPCR can only target one locus at a time. As large disease studies based on whole genome sequencing are just beginning, it is likely that the field of mCNV associations will move towards a genome-wide model, much like SNP associations did 10 years ago with the advent of arrays and GWAS methodology. Though there are notable genome-wide CNV studies done with arrays ${ }^{57,179}$, read-depth algorithms like Genome STRiP will most likely become the genotyping method of choice for mCNVs, because they allow for genotyping refinement, in that certain parameters can be adjusted and optimized until the copy numbers cluster at integers.

Yet, a gestalt approach that accounts for the structural haplotypes and SNPcorrelations of the mCNVs will likely still be necessary. mCNVs can contain elements that frustrate both computational and PCR-based approaches, such as stretches of extensive homology and varying breakpoints, elements that can be found and accounted for by the analysis of alleles. The SNPs in the vicinity of mCNVs also offer substantial information that is generally unexploited. While it is true that an individual SNP cannot serve as a proxy for a multi-allelic variant, it is also likely that the SNPs maintain some correlation between the copy number and/or haplotypes of an mCNV. As a result, a SNP's ability to capture an mCNV should not be thought of as a binary 'true' or 'false', but as a continuum. With some phenotype cohorts having as many as 120,000 individuals ${ }^{94}$, even a SNP with a low $\mathrm{r}^{2}$ can provide information on an mCNV's association.

Querying surrounding SNPs and SNP haplotypes can be used as a preliminary check for association in candidate gene studies, and upon completion of a genome-wide 
catalog of SNP to mCNV associations, for assaying mCNVs genome-wide in a hypothesis-blind manner. This analysis method would allow the field to make full use of current patient cohorts and avoid the time-consuming process of measuring the copy number of single candidate genes with PCR-based technologies. These computational results would likely have to be followed by direct measurement of the copy number of the $\mathrm{mCNV}$, but they can serve as a preliminary check to ensure that the disease field's resources are being focused on the most promising candidates.

This study was done with a focus on mCNV disease associations, but these methods could also be applicable to evolutionary biology. Being able to accurately measure the copy number of an mCNV across species, or even across time, may help unravel the evolutionary history of a species or genera. It may be that some mCNVs duplicated in response to the same selection pressure in separate species, resulting in convergent evolution - as in the case of amylase duplications in domestic dogs and humans ${ }^{18,147}$. Using optical mapping or other methods to assess mCNV haplotype structure may also be used to glean insights into how the mCNV, itself, evolved into its many forms. This could, perhaps, lead to a better understanding of mutational mechanisms, such as in the case of amylase in humans, where the duplications appear to have not have originated from the known mutation mechanisms. 


\section{Future Directions}

\section{What locus next?}

Someone seeking to enter the field of mCNV associations may ask which locus I recommend studying next. I could answer by pointing out loci that are expected to have a phenotypic effect because they contain genes that function in immunity, metabolism, development, the nervous system, or outward appearance. I could also point out genes that have a wide range of copy numbers, which could imply recent selection. However, following these suggestions means using a candidate-gene approach, in which there is a declared hypothesis that a particular gene influences a particular phenotype (or set of phenotypes). Though candidate gene studies have done much in the past, the field of human genetics has been moving towards a hypothesis-blind, genome-wide approach.

To this end, I would recommend a hypothesis-blind approach for finding the next mCNV to study. It is now possible to globally determine which SNPs most correlate to the copy numbers of $\mathrm{mCNVs}^{16}$. I would cross-reference the most correlated SNPs with GWAS databases to determine their P values for phenotypic associations. After crossreferencing, I would search the SNPs (binned by the mCNV they correspond to) for an enrichment in lower $\mathrm{P}$ values for any given phenotype, suggesting that the $\mathrm{mCNV}$ may be driving the association. From this, I would assemble a list of potentially phenotypicallyinfluential $\mathrm{mCNVs}$ that could be investigated further. 


\section{Technologies}

As hinted above, locus-specific techniques like qPCR and ddPCR will likely be supplanted by genome-wide approaches such as genome sequencing. With each year, more cohorts of genomes are becoming available, though many are only exomesequenced. It is easy to assume that exome-sequencing is not suitable for algorithms like GenomeStrip, because the selection process would distort the ratios of reads originating from a CNV versus the surrounding genome. However, it is possible that, though precise measurements may be impossible, there could still be a correlation between read depth and copy number. Across large numbers of samples, this correlation may provide information on the relative copy number status of the individuals.

Genome sequencing and other genome-wide techniques will still need secondary technologies for validating results and addressing technical limitations. Fiber FISH and optical mapping will likely be needed to assemble structural haplotypes, and ddPCR can target smaller regions that are difficult to assess with read depth. Even in situations where a single technology may suffice, a second technology should still be used to validate the initial results. An apt example would be Falchi et al.'s qPCR results. Though they had replicated their genotype calls with other technologies, they had used so few samples in the replications $(<96)$ that it was not apparent how imprecise their qPCR really was.

\section{Quality standards}

Having a second technology evaluate and replicate the results of the first technology should be a strived-for quality check, but it is not the only quality standard 
that should be used in future studies. To increase confidence in the association results, the copy number genotypes should cluster at integers (and confirm a lack of confounding factors) and SNPs should be utilized in the association as a positive control (if surrounding SNPs are correlated with the mCNV, then they should be modestly associated with whichever phenotype that $\mathrm{mCNV}$ is).

However, quality standards should extend beyond study design and into study reporting. At a minimum, the genotyped copy numbers should be clearly displayed within a figure of the paper, in a way that makes assessing their precision simple (no rounding the calls, masking the copy numbers with a box plot, or using a cryptic axis name or log scale). Ideally, all of the copy number calls would be published in the supplemental methods, though ethics and confidentiality agreements may make that impossible. In addition, the genotyping protocol should also be available, not only stated in paragraph form within the Methods; this ensures that no changes to the manufacturer's instructions are left accidentally unstated and that the reader does not miss an important point that was buried in a paragraph.

In the end, the final step of quality control lies with the reviewers and the readers. As a field, we need to be educated and vocal about the need for quality mCNV studies. We need to hold mCNV studies to the same standards we now use for SNPs and simple CNVs. We should also be open to the fact that, though there are controversies, there is a correct answer that we need to find. We cannot assume the original authors are correct, and we cannot assume that all controversial results are wrong. Together, we can investigate the mysteries of the human genome and potentially find therapeutic targets for devastating diseases. 


\section{Appendix A. Dissecting the timing \& origin of gene expression}

\section{variation}

\section{CONTRIBUTIONS}

Tom Mullen: Provided the initial idea for the project and did the majority of the wet-lab work.

Jim Nemesh: Did all of the groundwork computational analysis, such as sequencing read alignments and $P$ value adjustments.

Christina Usher: Did most of the analyses for the results, such as allelic imbalance comparisons across genes and across the genome.

\footnotetext{
ABSTRACT

Human gene expression variation contributes to the etiology of common diseases, yet it is unknown when and how these allelic differences in RNA abundance emerge. To address this, we developed a genome-wide approach (Het-Seq) to measure gene expression within lymphoblastoid cells using seven fractions representing different stages of the RNA lifecycle: nascent, early, nuclear, cytoplasmic, polyA, and ribosomal-bound RNA. We used a custom capture strategy and an informatics pipeline to quantify the RNA abundance from each allele of each gene, allowing us to measure the timing and spatial origin of expression differences. We found that $\sim 75 \%$ of variation is already present in nascent RNA, indicating a large role for transcription and a small, prominent role for post-transcriptional mechanisms. Overall, the allele expression differences were modest (less than 1.6 fold), but influenced $20-24 \%$ of the genes and correlated with local
} 
genetic variation $\left(\mathrm{r}^{2}\right.$ of 0.82$)$. These results indicate an extensive opportunity for new mutations and inherited polymorphisms to modify gene expression.

\section{INTRODUCTION}

Genome-wide association studies (GWAS) have mapped hundreds of traits to thousands of single nucleotide polymorphisms (SNPs) and the haplotypes those variants form ${ }^{180}$. While more than $80 \%$ of phenotype-associated haplotypes overlap with proteincoding genes, fewer than $15 \%$ contain a protein-altering allele ${ }^{181}$. This implies that intronic, synonymous, intergenic, and other untranslated variants are affecting phenotypes, most likely through gene expression. Supporting this idea, several studies have shown that, within a disease-relevant tissue, inter-individual variation in mRNA abundance tends to associate with the same SNPs as the disease $\mathrm{e}^{28,33,182-185}$.

Allele-differential expression (ADE) studies, which compare the expression of alleles within an individual, have estimated that $4-30 \%$ of genes have unequal expression $^{94,186-189}$, and expression quantitative trait loci studies (eQTL studies) have found many cis variants that could be causing this $\mathrm{ADE}^{33,182,190-195}$. But eQTL studies are not well-powered to detect trans effects on mRNA levels and cannot measure the impact of epigenetic variation that is not genetically controlled such as imprinting and random monoallelic expression ${ }^{196-199}$.

In addition, it is unknown when in the RNA lifecycle these variants alter expression. Transcription seems to be a key step, with genetic variants affecting transcription factor binding and epigenetic variants influencing the openness of chromatin $^{183,184,200-203}$, yet SNPs influencing splicing, polyadenylation, decay, and 
miRNA have been shown to affect expression levels, whereas other mechanisms, like nuclear export and translational regulation, have been understudied ${ }^{204-206}$. There is most likely a temporal distribution of effect, with some variants affecting transcription and others affecting post-transcriptional processes. This distribution could be determined using an ADE or eQTL study, but previous studies have used total mRNA - thus, mixing the effects of transcription, splicing, polyadenylation, and decay into a single measurement of RNA abundance.

We determined when variants exerted their influence over the lifecycle of RNA and whether those variants were solely genetic. We used two human lymphoblastoid cell lines (LCLs) from the 1000 Genomes Project - GM12878, a female with European ancestry, and GM19240, a female with Yoruban ancestry ${ }^{207}$. We isolated specific fractions of their transcriptome - nascent RNA (maximum of 5 minutes old), early RNA (60 minutes old), nuclear RNA, cytoplasmic RNA, polyribosome-associated RNA, total mRNA, and total RNA. We took the ratio of the two alleles present for each gene and charted the emergence of allele-differential expression spatially within the cell (using the nuclear and cytoplasmic fractions) and temporally over the lifecycle of RNA. Though this experiment cannot map the variants affecting a gene, it can identify when those variants act, hint at the regulatory mechanisms they modify, and determine whether they tend to be local and genetic.

\section{RESULTS}

\section{Experimental dissection of the maturing transcriptome}


RNA derived from whole cells reflects the influences of many regulatory mechanisms, including those that act upon transcription, splicing, nuclear export, translation, and mRNA stability. To parse the influences of different regulatory mechanisms, we first dissected the transcriptome into biologically meaningful fractions (Figure A-1A), combining many earlier innovations in RNA analysis ${ }^{208-210}$. We purified nascent $R N A$ by metabolically-labeling new transcripts with a pulse of 4-thiouridine (4SU) for 5 minutes in live cells before RNA extraction. The 4SU-labeled RNAs were harvested by biotinylating the $4 \mathrm{SU}$ tag and then capturing on streptavidin-coated beads. We similarly purified early $R N A$ by 4 SU-labeling for 60 minutes before extraction. We separated nuclear RNA from cytoplasmic RNA by hypotonically swelling living cells and dounce-homogenizing the resulting suspension, then centrifuging at a force sufficient to pellet nuclei. We further fractionated the cytoplasmic RNA to identify actively translating, polyribosome-associated $R N A$ by ultracentrifugation through sucrose gradients, eluent collection, and identification of the polyribosome-containing fractions. Within each fraction, we sequenced total RNA without poly(A) selection, after depleting fractions of ribosomal RNA (rRNA). For comparison to more conventional RNA sequencing techniques, we also sequenced total $m R N A$ and total $R N A$.

To evaluate the integrity of each cellular RNA fraction, we analyzed the RNA sequences using both genome-scale and locus-specific analyses (Figure A-1B). At the genome scale, the maturity of RNA can be monitored from the portion of RNA arising from intergenic, intronic, and exonic segments of the genome as currently annotated (albeit imperfectly) in the human reference sequence. In genomic DNA, 55\% of sequence reads arose from annotated intergenic regions, and only $1-2 \%$ from annotated exons, 
consistent with the size and number of these features in the reference genome sequence $^{211}$. In nascent RNA, only $8 \%$ of sequence reads arose from intergenic regions, while $70 \%$ arose from annotated introns. This was followed, as the transcriptome matured, by a gradual increase in the contribution of annotated exonic sequences, and a concomitant decrease in the contribution of annotated intronic sequences, reflecting processes of RNA maturation, splicing, and intron degradation. Total mRNA corresponded to the most mature of these fractions, while total cellular RNA appeared to be a mixture of all of them. 


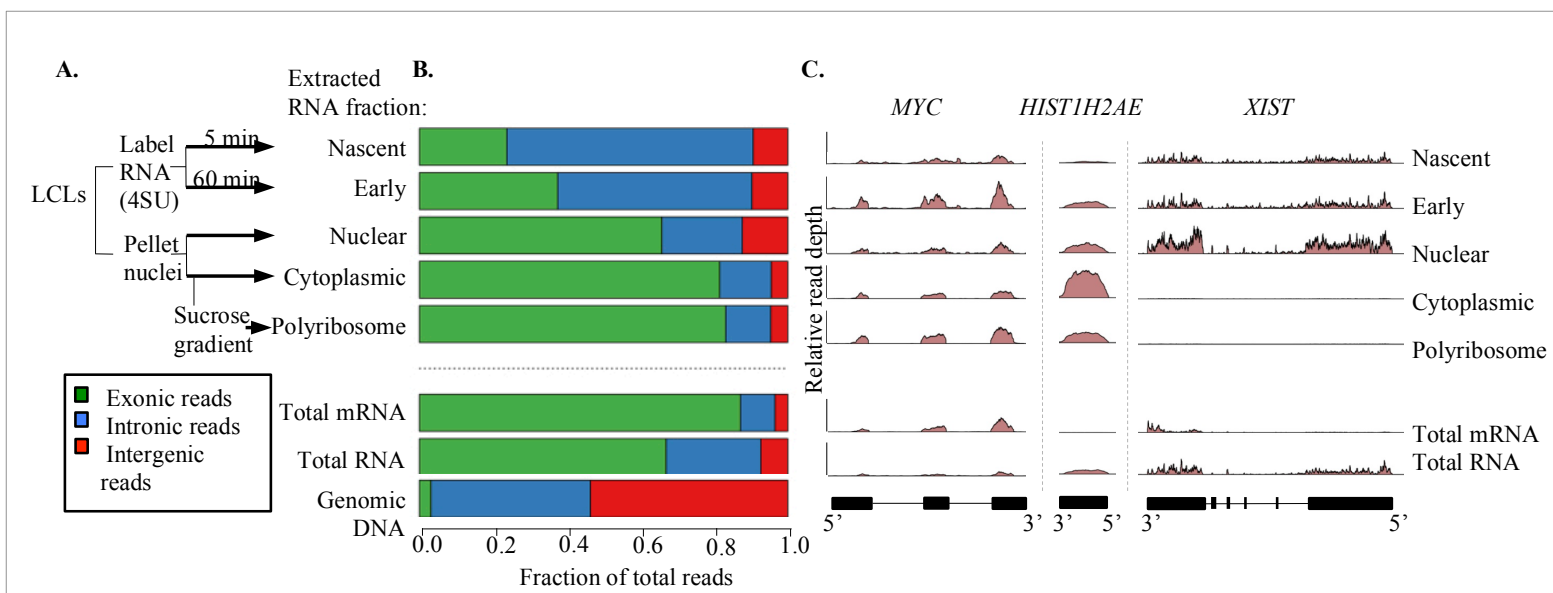

Figure 1. Efficient isolation of important fractions of the transcriptome. (A) The

method of extracting important fractions of the transcriptome - nascent, early, nuclear, cytoplasmic, and polyribosome-associated RNA transcripts. (B) The proportion of sequence reads arising from exons, introns, and intergenic regions for each transcriptome fraction. Maturation of the transcriptome is apparent with the loss of intronic reads. (C) Maturation of RNAs from example genes. Normalized read depth is plotted in red and binned in 25-bp segments over the gene structures in black. Our results agree with the current knowledge that $M Y C$ encodes a typical mRNA transcript, HISTIH2AE RNA is not polyadenylated, and XIST encodes a nuclear-retained RNA.

We further evaluated these transcriptome fractions utilizing genomic loci whose transcriptional biology is well understood (Figure A-1C). Most multi-exonic, proteincoding genes produced sequence reads across their exonic and intronic regions in nascent RNA, then matured to exon-only profiles in mature RNA fractions. Transcripts with eccentric, and well characterized, life cycles also showed the expected behaviors in our transcriptome fractions. For example, XIST RNA, which is retained in the nucleus ${ }^{212}$, was 
abundant in nascent, early, and nuclear RNA fractions, but absent from cytoplasmic and polyribosome-associated fractions. Histone-protein-encoding RNAs, which are not polyadenylated and are transcribed at low levels but accumulate to high abundance due to low turnover in dividing cells ${ }^{213}$, showed low relative expression in nascent RNA, greater abundance in more-mature fractions, and absence from the total mRNA fraction. As expected, polyA-selected mRNAs showed a 3' bias, but our compartmentalized RNA fractions (which were sequenced in total, without poly(A) selection) did not.

Thus, this series of RNA fractions form a five-scene movie of the maturing transcriptome. The next step was to identify the scenes in which allele-specific effects appeared. We sought to understand to what extent regulatory sequence variation shapes each fraction, and what distribution of large and small effects such variants encode.

\section{Het-Seq: Measuring allelic effects with precision and accuracy}

To monitor allele-specific regulatory effects in a precise and well-controlled way, we utilized the heterozygosity that exists within each diploid genome to separately follow the expression of homologous copies of each gene (one derived from each parent) (Figure A-2A). Alleles of the same gene in the same individual are potentially powerful controls for each other, as both are subject to the same trans-acting genetic and

environmental influences ${ }^{214}$. Cis-acting genetic variation can cause alleles to diverge from the expected 1:1 ratio, displaying "allelic skew" or "allelic imbalance". 
A.

B.
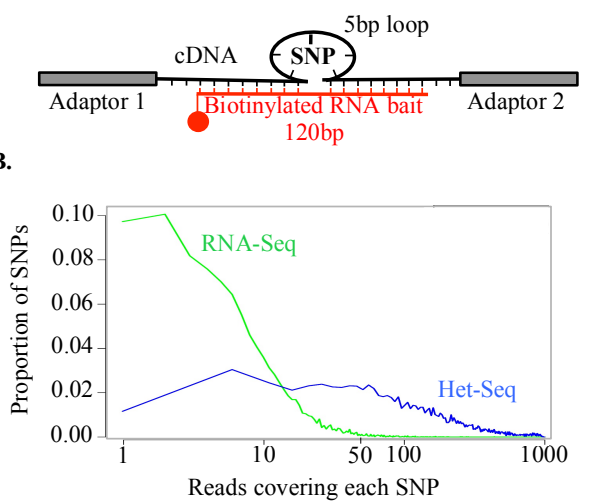

D.

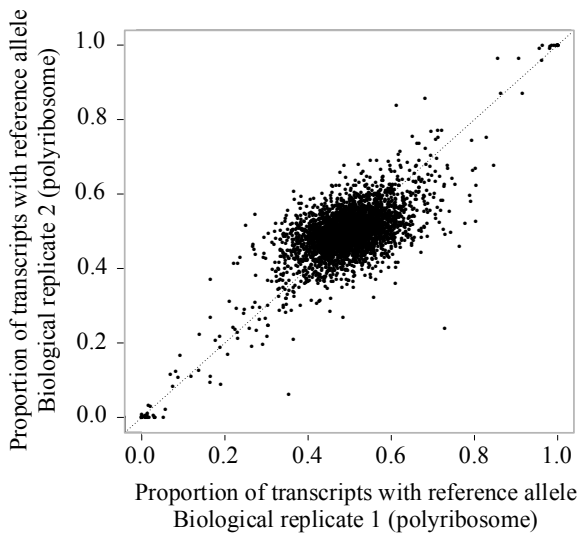

C.

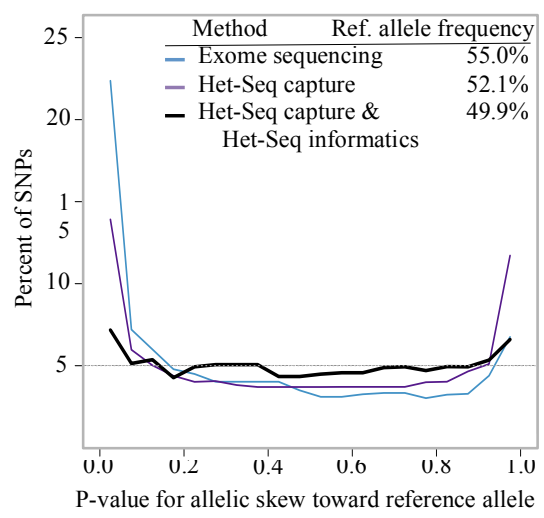

E.

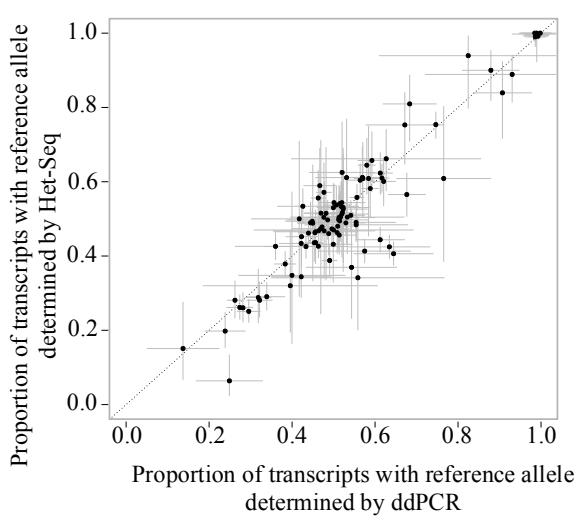

Figure 2. Equal capture of alleles allows precise measurements of AR. (A) The

Het-Seq capture strategy allows the equal capture of cDNA from both alleles by using RNA baits that bind the non-variable flanking sequence around each SNP.

This capture strategy is effective for increasing coverage on sites of interest. (C)

SNPs in genomic DNA exhibit a nearly uniform distribution of p-values when captured with Het-Seq probes and analyzed with Het-Seq informatics. (D)

Biological replicates showed a good concordance for both modest and large allelic skew events. (E) Confirmation of a subset of Het-Seq measurements was done on the ddPCR platform. 
Allelic ratios (AR) in RNA can be obtained by RNA sequencing, which has previously been used to detect the extreme forms of allelic skew found at imprinted $\operatorname{loci}^{147}$ and to validate eQTLs ${ }^{192,193}$. Standard RNA sequencing, however, is not sensitive enough to monitor modest allelic skew in most genes. Because the expression levels of different genes vary across many orders of magnitude, a few highly expressed genes consume much of the bandwidth of a sequencing experiment (Figure A-2B). Thus, while a few genes may achieve average read depth of 100 reads or more across their length, allowing measurement of ARs with a precision of $+/-10 \%$, the vast majority of hetSNPs are interrogated by far fewer sequencing reads, allowing the detection of only extreme allelic skews in individuals (such as those present at imprinted loci) and requiring averaging across many individuals to detect more subtle skews. Enriching RNA-seq libraries for sites of interest can be done with PCR, arrays, or padlock probes ${ }^{147}$, but such approaches have tended to distort the relative representation of pairs of alleles or to obscure the extent to which measurements derive from PCR duplicates.

To measure allelic ratios across the transcriptome with high precision and accuracy, we developed an approach, Het-Seq, for enriching RNA-seq libraries for heterozygous sites while preserving allelic ratios and information about the molecular origin of each sequence read. In Het-Seq, we first computationally design thousands of "enrichment probes" to the sequences flanking hetSNPs (Figure A-2A). The probes contact only invariant sequence close to the hetSNP, so that the allelic state of the SNP does not influence the avidity of capture. These biotinylated probes are then used to capture and enrich RNA-sequencing libraries for molecular inserts containing the informative SNPs. A custom informatics strategy aligns the resulting sequence reads to a 
reference genome on which heterozygous sites have been masked out, making sequence reads with the reference and alternative alleles equally likely to align to that genomic location. Potential PCR duplicates are systematically removed from analysis, ensuring that each allelic count represents a distinct observation of the original first-strand cDNA. In this way, we obtain an integer measurement of the relative abundance of each allele in the original RNA sample. If a read equally matches more than one site (as in the case of $\mathrm{CNVs}$ ), it is aligned randomly to one site. The digital nature of this count, and its derivation from independent reverse-transcriptase events, allows the significance of AR measurements to be evaluated using binomial statistics.

Het-Seq dramatically enriched RNA-seq libraries for reads that were informative about the states of heterozygous SNPs, allowing thousands of hetSNPs to each be evaluated for AR with more than 100 molecularly independent observations on each one (Figure A-2B).

\section{Critical evaluation of Het-Seq}

It was important to critically evaluate the extent to which Het-Seq was susceptible to artifacts that would undermine the measurement of allele-specific cis-regulatory effects. Such artifacts can be discerned by analyzing genomic DNA, in which each pair of alleles is equally abundant, allowing robust detection of technical influences on measurement.

The first potential problem involves systematic bias toward alleles present in the reference human genome sequence. Such "reference bias" arises wherever pairs of alleles are captured with unequal avidity or are aligned to the reference genome with unequal 
efficiency (for example, because the alternative allele is disadvantaged by already containing one mismatch to the reference sequence). Supporting the idea that these are important effects, we found that exome sequence data (from NA12878 and NA19240 in the 1000 Genomes Project) contained the reference allele in $55 \%$ of aligned sequence reads. By contrast, in Het-Seq, the reference and alternative alleles were ascertained equally (49.9\% reference, $50.1 \%$ alternate). About three-fifths of this improvement appeared to arise from Het-Seq's allele-neutral molecular capture strategy, with the rest coming from a custom informatics pipeline that neutralized sources of reference-allele bias (Appendix B).

The second potential problem comes from the tendency of PCR to create duplicate copies of the sequences in molecular libraries. If scored and treated as independent observations, PCR duplicates cause measurements of allelic ratio to be overdispersed - to tend toward extreme ratios - relative to a null hypothesis in which allelic counts arise purely from random statistical sampling. The resulting $P$ values would be systematically lower, with the result that the breadth and magnitude of allelic influence would be overestimated. Because Het-Seq involves paired-end sequencing of a molecular library with diverse molecular sizes and offsets relative to each SNP, we can filter all data points that are potential PCR duplicates (those with the same start and end location on the genome) while maintaining those observations that are molecularly independent. This ensures that each allelic count represents a distinct observation of the original first-strand cDNA.

Encouragingly, allelic imbalance $P$ values for genomic DNA showed a uniform distribution similar to the distribution expected under the null hypothesis (of random, 50- 
50 sampling of each pair of alleles) after allele-neutral capture and Het-Seq informatics analysis (Figure A-2C). Though there is still a minor inflation, the effect sizes of these skews are small and disappear in the RNA libraries, which have a lower read count (Discussed in Appendix B. Experimental Procedures).

Because we were analyzing highly manipulated biological samples, it was critical to understand the extent to which AR measurements reflect durable features of the underlying biology. To evaluate this, we grew the cells in distinct experiments months apart, harvested RNA at distinct times (also months apart), and processed the samples separately. AR measurements were reproducible across these biological replicates. Notably, the high correlation of AR measurements was observed not only for genes with extreme measurements of allelic skew, but for genes that showed modest allelic skewing of 10-50\% (Figures A-2D).

With our precision confirmed, we tested our measurements' accuracy. We selected 20 SNPs covering a range of Het-Seq allelic skews and assayed them with allelespecific droplet digital PCR across the RNA fractions and individuals. This created over 100 independent calls that were broadly concordant with Het-Seq (Figures A-2E). Thus, we believe this is the first study of allele-differential expression that has simultaneously met the criteria of (i) deep ascertainment of heterozygous sites, (ii) allelic neutrality during detection, (iii) dispersion statistics that closely match random sampling, (iv) precise, accurate measurements of even modest allelic skew, and (v) independent measurements across the lifecycle of RNA. 


\section{An allelic-skew profile acts as a fingerprint of genome identity}

These data allowed the detailed profiling of a transcriptome in terms of the quantitative influences of genome sequence variation on thousands of genes. Though the causal sequence variants are unobserved, their direct effects are measured via the systematic use of sequence variation on nearby transcripts to monitor allele-specific regulatory effects in an internally controlled way.

We sought to understand the global properties of these genome-wide profiles of allelic skew. We found that when we used the RNA-seq data to measure absolute gene expression levels in nascent, early, nuclear, cytoplasmic, polyribosome-attached, and mRNA fractions, these genome-wide profiles clustered by the RNA fraction from which they were derived (rather than the individual from whom we derived them), reflecting substantial differences among the fractions in the abundances of different transcripts. However, when we clustered Het-Seq allelic-ratio profiles derived from these same RNA libraries (limiting analysis to 1,795 hetSNPs that were heterozygous in both probands and deeply interrogated by $>100$ reads in each), AR measurements clustered by the genome of the individual from whom they were obtained, reflecting the unique set of unseen regulatory variants in each individual (Figure A-3A). 
A.

By gene expression:

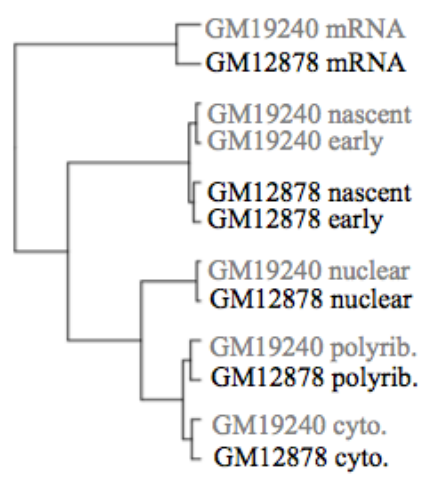

By allelic skew:

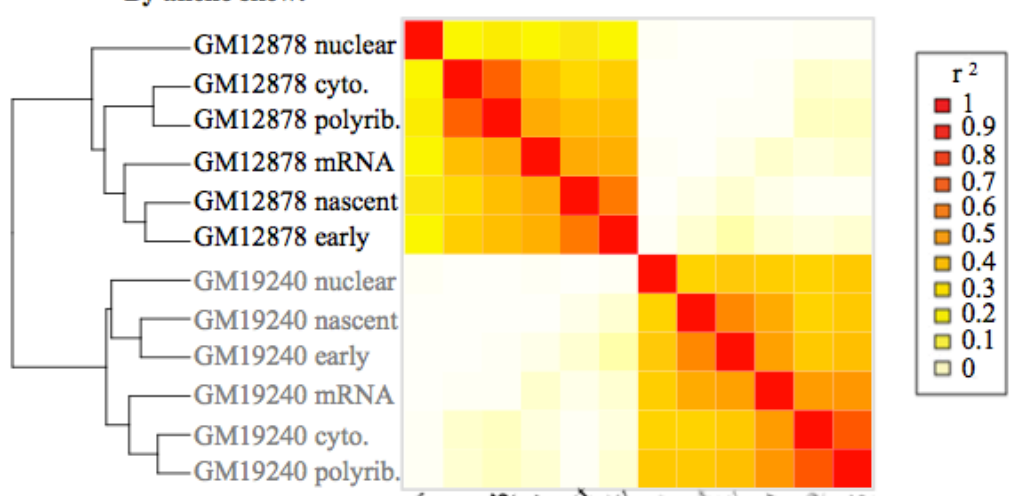

a

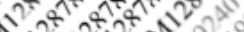

तो

B.

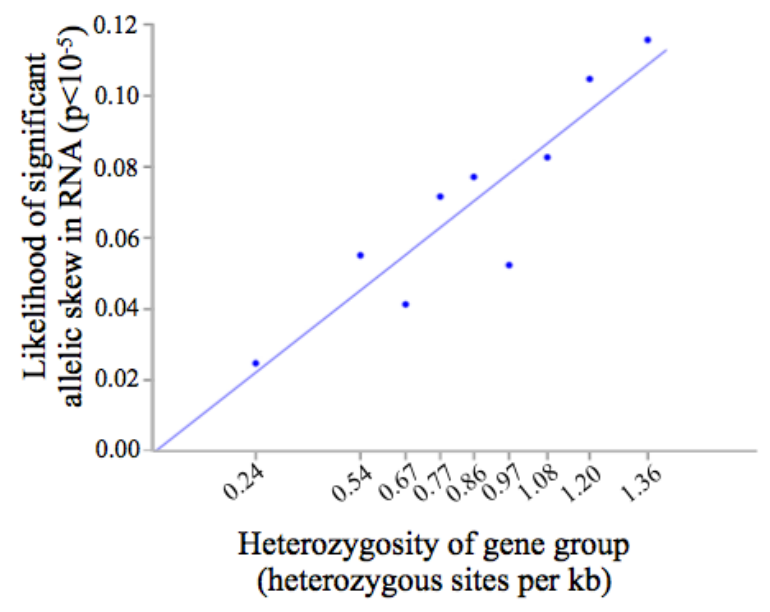

Figure 3. Allelic skew correlates with genetic variation. (A) Allelic skew profiles cluster by individual, even when gene expression profiles tend to cluster by fraction. Analysis was limited to captured SNPs that were heterozygous in both individuals (1795 SNPs with $>100$ reads). (B) To identify whether the allelic skew profile of an individual was due to genetic variants, we correlated the likelihood of a SNP showing skew with the heterozygosity around that SNP. There was a strong correlation with an intercept of nearly 0 , suggesting that the majority of the skews have a genetic basis. 
We sought to determine whether the regulatory variants were genetic, or from other non-genetic forces such as random or programmed monoallelic expression ${ }^{196}$, or subtle, technical biases not detected in the above evaluation. To distinguish between these hypotheses, we developed a test that utilizes the mosaic distribution of heterozygosity in a typical human genome. Each diploid human genome is a mosaic of segments of low and high heterozygosity, ranging from regions where one's maternal and paternal haplotypes are very similar, to regions where they have many sequence differences per kilobase. In each sample, we divided all protein-coding genes into ten groups (deciles) based on the level of heterozygosity at each locus, from the most-homozygous loci to the most-heterozygous loci. The heterozygosity present in each decile strongly predicted the tendency of its genes to exhibit definitive allelic skew (Figure A-3B). This relationship was linear with a y-intercept of almost zero. This result suggests that allelic skewing in RNA arises overwhelmingly from genome sequence variation, rather than from other mechanisms. 
A.

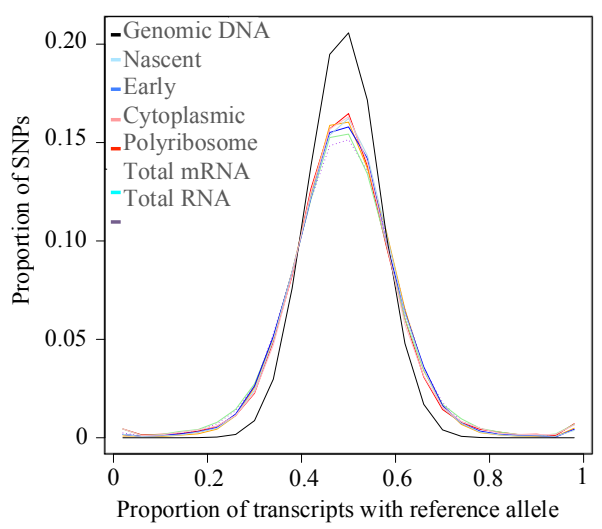

C.

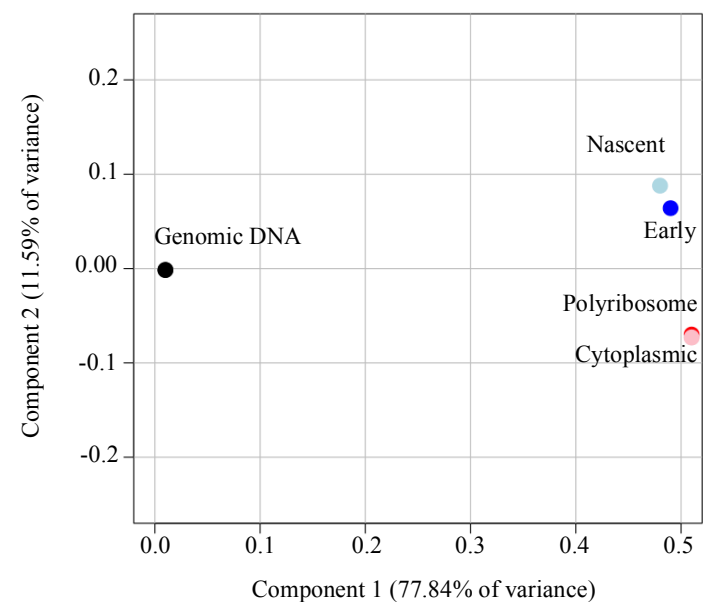

B.

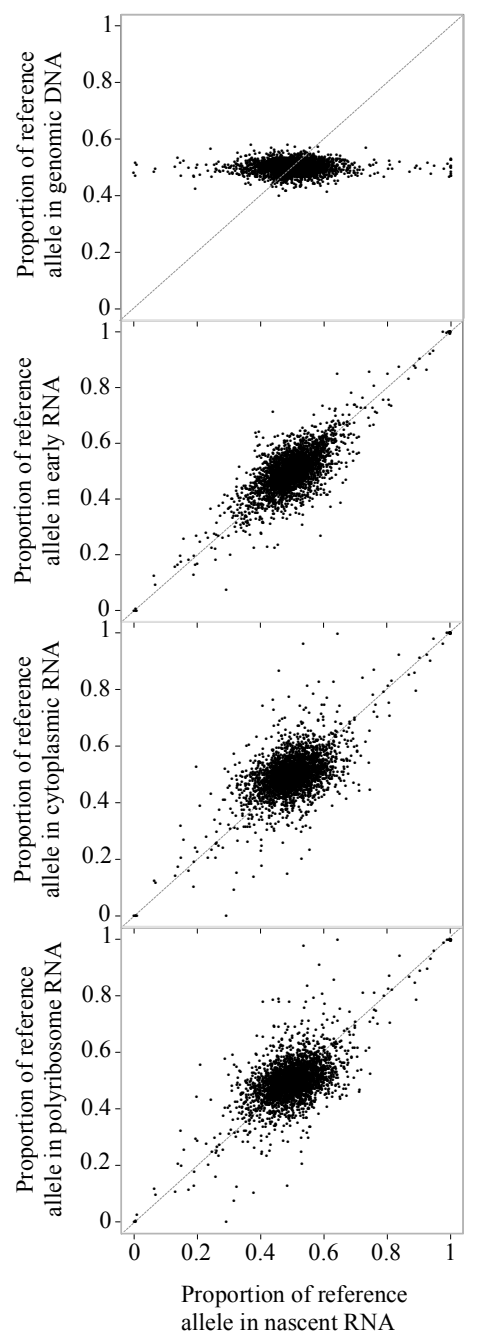

Figure 4. Most allelic skews arise at transcription. (A) Distribution of allelic skew across phases of RNA maturation in GM19240. SNPs included in the analysis were present in all fractions with greater than 50 reads. In order to equalize the distribution-slimming effects of high coverage in some fractions, allelic counts for each SNP were down-sampled to 50 before plotting. (B) Pairwise comparison of each SNP's allelic skew. (C) Principal components analysis of allelic skew profiles. 


\section{Allelic skew affects $20-24 \%$ of genes and is modest in magnitude.}

Het-Seq measurements of

allelic ratios in each RNA sample

showed a distribution substantially

wider than the distribution derived

from analysis of genomic DNA or

expected from random statistical

sampling of pairs of alleles (Figure

A-4). The observed variance in

allelic-ratio measurements is a

composite of (i) true regulatory

influences, and (ii) the effect of

random ("binomial") statistical

sampling upon each measurement.

To parse these effects and infer the

true distribution of regulatory effect

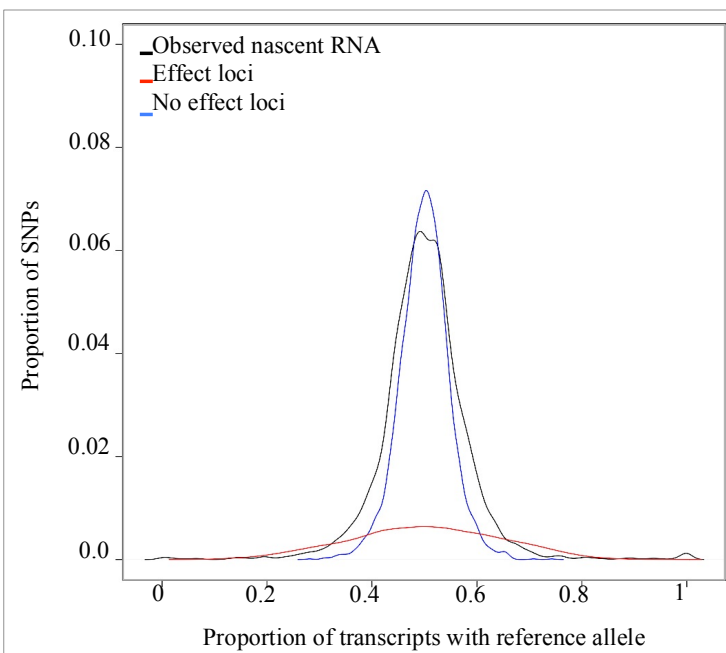

Figure 5. Allelic skew is common and modest in RNA

Beta-binomial analysis splits the observed distribution into a distribution of real effects and a distribution of null effects. It estimates that $24 \%$ of genes exhibit a skew with a median effect size of 1.5 -fold $(60: 40)$ in GM19240.

sizes, we used beta-binomial

statistics, which model the data as arising from both an underlying distribution of real regulatory effects (affecting a subset of genes, with effect sizes exhibiting a beta distribution across these genes), and the influence of binomial sampling upon all measurements. We found that beta-binomial statistics fit the experimental data well, and used the beta-binomial model to estimate two key parameters: the fraction of genes affected by allele-specific influences, and the distribution of regulatory effect sizes. 
The model estimated that $\sim 24 \%$ of genes in GM19240 displayed alleledifferential expression with a median influence of 1.5 -fold, corresponding to an allelic ratio of $60: 40$ (20\% of genes at 1.6-fold for GM12878). In fact, $80 \%$ of the effects changed expression 2.0-fold or less (2.1-fold for GM12878). This is mostly consistent with a recent study that found the majority of mutations within mammalian enhancers influenced activity less than 1.5 fold, with only $3 \%$ of mutations affecting activity by more than two-fold ${ }^{215}$. Our effect sizes are higher, probably due to the additional variation expected to occur within an individual's enhancer, promoter and transcript that may influence expression.

\section{How expression variation affects individual genes.}

Once we had characterized genome-wide expression variation, we documented how this affected particular genes. A majority of genes showed no apparent allelic influence - the maternal and paternal alleles were equally abundant in nascent transcripts and at every subsequent stage of RNA maturation, export, and processing. Four examples are shown in Figure A-6A. 
A. No allele-specific regulation:

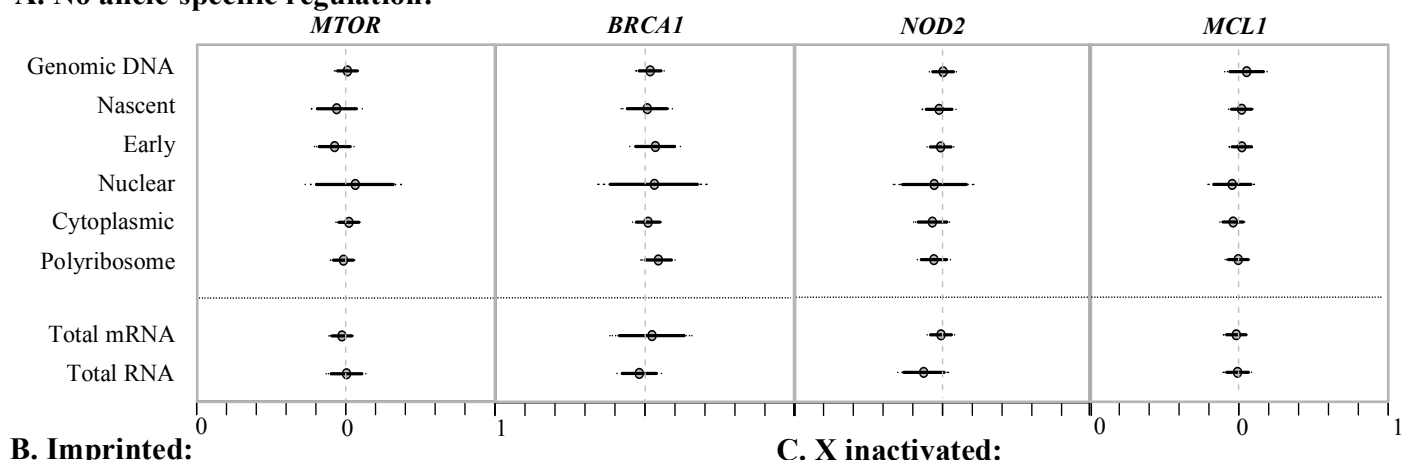

B. Imprinted:

C. $X$ inactivated:

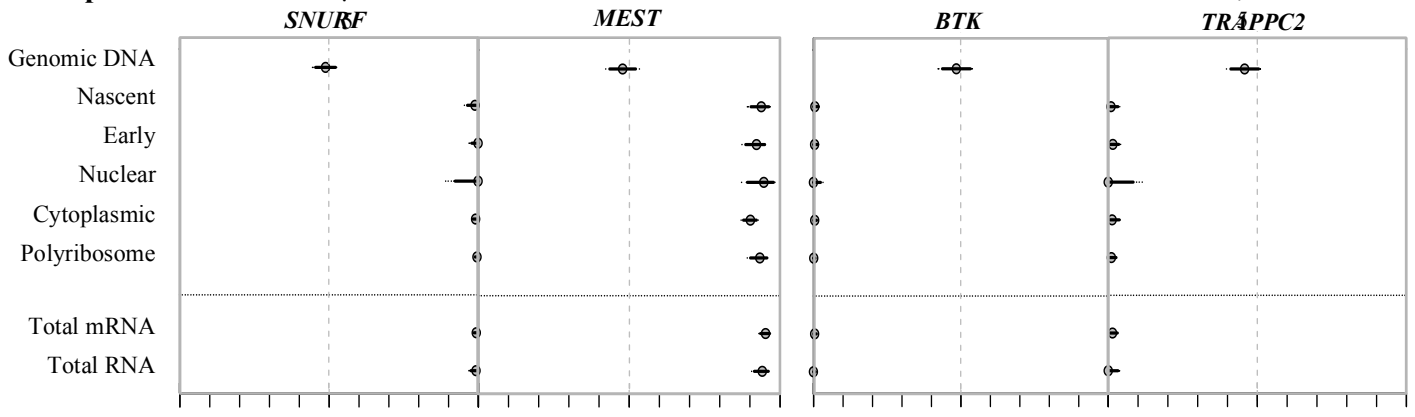

D. Allele-specific regulation at transcription:

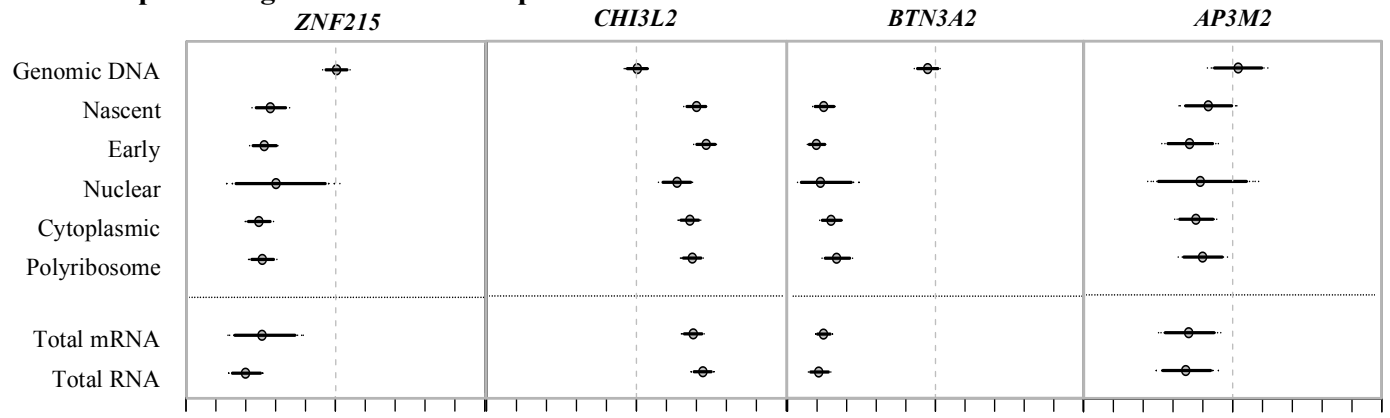

E. Allele-specific regulation after transcription:

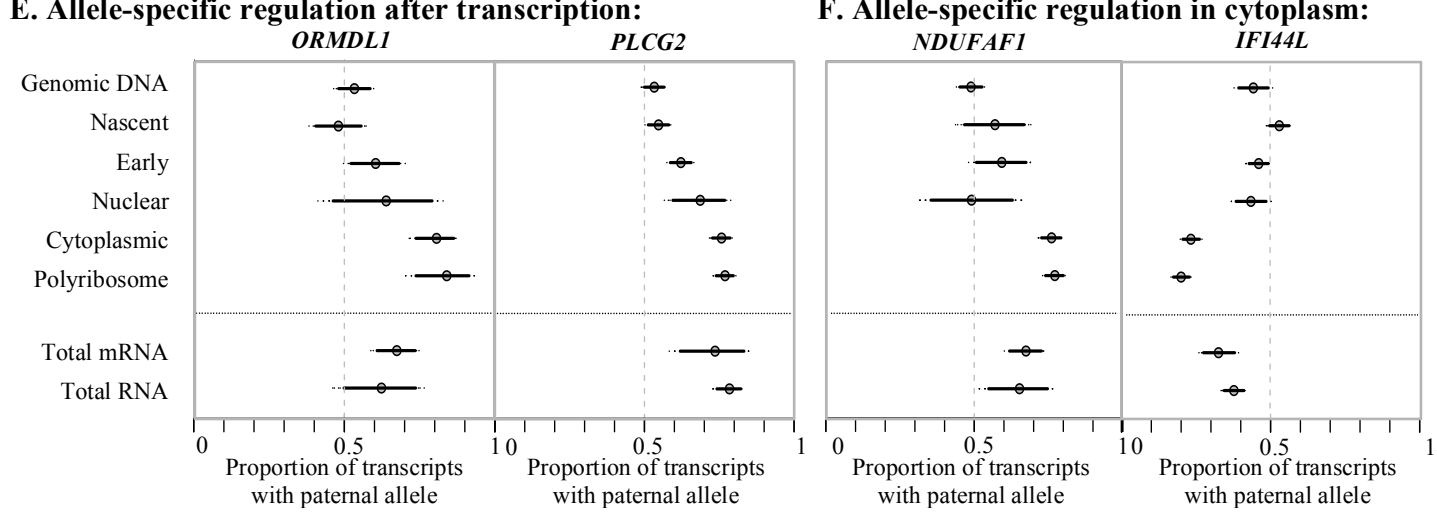

Figure 6. Examples of different allelic skew profiles. Most genes are like those of (A),

with no effect. However, a substantial fraction are like those in (D), where an effect manifests at transcription and persists through RNA maturation. Rarely, an effect manifests in the cytoplasm, presumably from a cytoplasmic-specific RNA maturation step. 
We documented extreme allelic skew from known imprinted such as $S N U R F$, a paternally imprinted gene residing in the Prader Willi locus on chromosome 15q11, and MEST (also known as PEG1), a paternally imprinted gene residing on chromosome 7q32 ${ }^{216}$. Notably, some imprinted genes, such as MEST, showed incomplete inactivation of the non-favored allele, producing as much as $5 \%$ of the transcripts from the gene. We also found new genes that fit the pattern of being imprinted (TMEM173, MACC1, AL662904.1, and FHOD3), though FHOD3 and MACC1 were only skewed in one individual. As expected, most genes on the X chromosome were inactivated. However, $M S L 3, Z F X, R B B P 7$, and $K D M 6 A$ escaped inactivation in both individuals. $Z F X$ is an important, dosage-dependent cancer gene in non-small cell lung cancer ${ }^{217}$, but it could be an artifact of cell culture so more investigation is needed. In total, cases of overwhelming (>90\%:10\%) allelic skew were found in only $2.7 \%$ and $7.3 \%$ of skewed genes in GM12878 and GM19240, respectively - over half of which were on the X chromosome.

Modest allelic skew was far more common than extreme allelic skew. Consistent with the genome-wide analyses, the most common pattern was an effect arising at transcription. This pattern involved an allelic skew that appeared for the first time in nascent RNA, then persisted at a constant magnitude across all subsequent fractions (Figure A-6D). 61\% of classifiably skewed genes exhibited this pattern in GM12878 and $58 \%$ in GM19240. This number rises to $85 \%$ and $80 \%$, respectively, when genes with an additional post-transcriptional effect are included.

For some genes (7.8\% and 7.3\% of skewed genes in GM12878 and GM19240), the allelic skew appeared to grow steadily in magnitude as the transcriptome matured (Figure A-6E). Such observations were particularly common among genes with different 
isoforms, and tended to involve a hetSNP in an alternatively spliced exon. However, some of these could result from different rates of decay.

Other genes (4.7\% and 5.0\% of skewed genes in GM12878 and GM19240) exhibited allelic balance in all nuclear-resident fractions but strong skew in the two cytoplasmic fractions, suggesting that they are regulated in an allele-specific way by mechanisms that reside in the cytoplasm, such as miRNAs or nonsense mediated decay, or were blocked from the cytoplasm, perhaps by nuclear export mechanisms. Examples of these genes are the complex I assembly protein $N D U F A F 1$ and the interferon-induced family member IFI44L (Figure A-6F). Consistent with the hypothesis of cytoplasmic regulation, IFI44L had a heterozygous SNP in a predicted binding site for the LCLexpressed microRNA1206, and the abundance of the allele that better matched the mir1206 binding site was reduced in cytoplasmic RNA fractions.

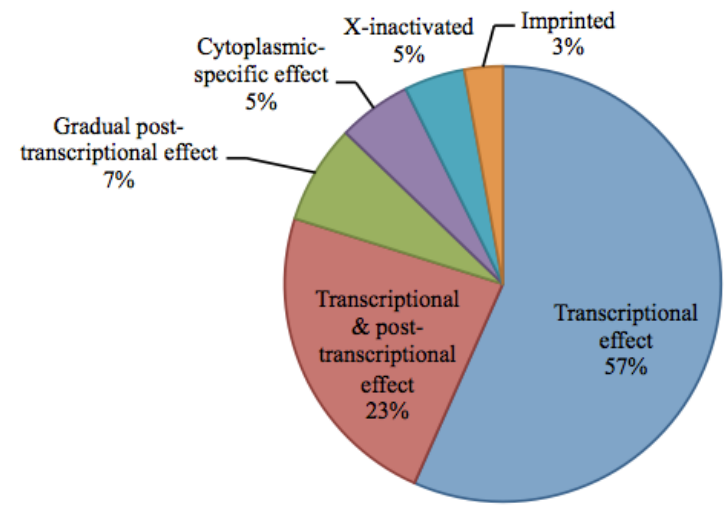

Figure 7. When allele-specific effects arise in the transcriptome of GM19240.

Genes with more than 50 reads in each fraction and at least one significant allelic skew were sorted into 6 different categories of allele-specific regulation (424 genes total). Even though this data is based on a small number of genes, it is concordant with the other genome-wide analyses. 
Overall, the classification of genes agrees with the genome-wide assessments of the impact of transcriptional and post-transcriptional effects. Transcriptional effects seem to dominate, yet there is a substantial effect produced by alternative splicing and a few convincing instances of cytoplasmic-based regulation (Figure A-7). Given that 20-24\% of genes exhibit these skews, there are many ways a variant can affect mRNA expression and thereby influence phenotype.

\section{DISCUSSION}

We described an approach for quantifying the effects of cis-acting allelic perturbations across the genome. Data from this approach are highly reproducible and robust to statistical tests of validity, and offer a promising, straightforward way to profile the effects of genome sequence variation upon most genes.

We anticipate that Het-Seq will have many other applications. One set of applications will involve further characterizing and mapping the regulatory perturbations ascertained in genome-wide association studies (GWAS). Critical directions in the biological exploitation of GWAS results include determining which gene(s) are affected at each locus, determining the sign and magnitude of allelic effects on each gene's expression level, and localizing these effects to specific populations of cells. By performing Het-Seq on cell-type-specific populations of RNA, regulatory effects in complex tissues could be systematically mapped to specific cell types, analogous to our mapping of such effects to compartments within cells of a single type. This will be useful for going from these early genetic leads to models of pathophysiology in complex tissues. 
Our results suggest that transcriptional regulation predominates among the influences of genome sequence variation on gene expression. Several potential reasons are suggested by the data in this study. First, transcriptional regulation appears to present a substantial mutational target - we estimate from the density of heterozygous sites (about one per 1,250 bp) and the frequency of allelic skew (about $20 \%$ of genes) in an individual genome that, even in a single cell type, the average gene contains at least 250 bp of sequence that can mutate to an expression phenotype. Such sequences could include not only enhancers but also others classes of transcriptional regulatory elements, such as insulators, repressors, pause sites, and matrix attachment regions. Second, transcriptional regulation appears to offer many opportunities for mutation to give rise to soft, quantitative effects on the expression level of a gene. We estimate from the mathematical distribution of allelic skews that most regulatory influences involve a less than 1.4-fold difference in the expression levels of the two alleles, and fewer than $10 \%$ involve a 1.8fold difference. Such quantitative changes may be well-tolerated in robust biological systems, allowing such functional variants to become common in populations and to contribute to variation that is generally within the healthy range. It is likely that constellations of many such variants in the same pathway may, in some individuals, push phenotypic variation to extremes within and outside the range defined as normal. The high evolvability of regulatory sequences may help us to understand why they account for the phenotypic variation that arises within species on short time scales. 


\section{Appendix B. Experimental Methods}

\section{Amylase}

\section{Cohort Collection:}

Estonian Sample Collection: The Estonian Biobank is the population-based biobank of the Estonian Genome Center of the University of Tartu (EGCUT) (www.biobank.ee). The EGCUT is conducted according to the Estonian Gene Research Act, and all participants are volunteers that have signed broad informed consent ${ }^{167}$. The cohort size is currently 51,535 people from 18 years of age and up. All subjects are recruited randomly by general practitioners and physicians in hospitals. A computerassisted personal interview is conducted at the doctor's office to record personal data, genealogical data, lifestyle data, and the subjects' educational and occupational history.

Medical history and current health status are recorded according to the ICD10 and medication according to the ATC. Anthropometric measurements are taken, along with blood pressure (sitting position at the end of the interview), and resting heart rate. 30-50 $\mathrm{mL}$ of venous blood are collected into EDTA Vacutainers. These are transported to the central laboratory of EGCUT at 4-6 degrees Celsius within 6 to 36 hours after collection. Upon arrival, DNA, plasma and WBC are immediately isolated and kept in aliquots in MAPI straws in liquid nitrogen.

A Hamilton Robotics Automated Sample Management system with a 100,000 tube capacity is used for intermediate storage of normalized DNA samples (50-100 $\mathrm{ng} / \mu \mathrm{L}$ ) in tubes with $2 \mathrm{D}$-barcodes. This enables a quick and highly accurate delivery of the samples by cherry-picking according to the selected barcodes. 
The 1,000 Estonian samples used in the current study were selected from the BMI extremes of the EGCUT population cohort of 51,535 samples. The lean $(\mathrm{BMI}<22)$ and the obese (BMI > 33) groups were matched on age-at-recruitment and year-of-birth to account for cohort and life-course effects on overall body composition, resulting in the obese being in the $98^{\text {th }}$ percentile (females: $99.5^{\text {th }}$ percentile) of the cohort's BMI and the lean being in the $87^{\text {th }}$ percentile (females: $82^{\text {nd }}$ percentile). The extreme sets included 250 samples from both genders and were limited to only individuals with Estonian nationality and whose self-reported mother tongue was Estonian. Further measures to account for potential population structure are described further down.

GoT2D Sample Collection: The Genetics of Type 2 Diabetes (GoT2D) study aims to characterize type 2 diabetes and related quantitative traits' genetic architecture through low-coverage whole-genome sequencing, deep ( 100x) exome sequencing, and 2.5M SNP genotyping of cases and controls from four large European cohorts: Diabetes Genetics Initiative (DGI), Finland-United States Investigation of NIDDM Genetics (FUSION), GoT2D-UK, and Kooperative Gesundheitsforschung in der Region Augsburg $(\text { KORA })^{13,218-221}$. These larger cohorts are a mixture of smaller ones obtained in Finland, Sweden, the UK, and Germany. Due to the confounding effect of diabetes on BMI, cases and controls were analyzed separately while controlling for the cohort of origin. Only the low-coverage data was used in the current study.

InCHIANTI Cohort Sample Collection: Individuals $(\mathrm{n}=680)$ were selected from the InCHIANTI study ${ }^{168,222}$ - a study of aging from the Chianti region in Tuscany, Italy - for low pass whole genome sequencing. Individuals were selected for sequencing 
based on the availability of gene expression and circulating biomarker data, though neither were used in this study.

Whole-genome sequencing was performed at the Beijing Genomics Institute (BGI), Shenzhen, China using the Illumina HiSeq 2000 to obtain a minimum read depth of $6 \mathrm{X}$. An average of 240 million paired-end 90bp reads per sample were aligned to the 1000 Genomes implementation of the Genome Reference Consortium's build 37 of the human reference genome $\mathrm{e}^{223}$, using the burrows-wheeler aligner (BWA) version 1.5.9. $9^{224}$.

GPC Cohort Sample Collection: Headed by the Center for Genomic Psychiatry at USC, the GPC cohort is a collection of individuals with schizophrenia and bipolar disorder, along with controls. Participants are recruited from the United States and selected sites abroad. The subset used in this study consisted of 768 self-reported (and genetically confirmed) European-ancestry patients from the United States that were whole genome sequenced from blood to a depth of 30x. Data on BMI were not available, so this cohort was used in the analyses determining the amylase structural forms and their relationships to SNPs.

\section{Droplet Digital PCR:}

General: Droplet digital PCR is similar in concept and preparation to a real-time qPCR reaction but with a few important modifications ${ }^{154}$. Before amplification, the DNA is first digested with a restriction enzyme in order physically separate the copies of the CNV that are on the same DNA strand (the enzyme is chosen based on the locus, but common choices include AluI, EcoRII, and RsaI). The PCR reaction mixture is prepared similar to qPCR, with each primer at $900 \mathrm{nM}$, the fluorescent probes at $250 \mathrm{nM}$, and the 
digested DNA around $1 \mathrm{ng} / \mathrm{ul}$. The reaction $(20 \mu \mathrm{l})$ is then emulsified into approximately 20,000 water droplets surrounded by oil using a droplet generator (BioRad). The droplets are thermocycled using a standard thermocycler with the BioRad-supplied PCR protocol (with an additional 10 cycles), and the droplets containing the probes' targets then become fluorescent. The fluorescent droplets are then counted by a droplet reader (BioRad). At low DNA input concentrations, each fluorescent droplet likely contains only one PCR target, thus allowing us to count the near-exact number of targets within the reaction - as opposed to comparing amplification curves, like in qPCR. A Poisson correction factor is applied by the software to account for droplets that contain more than one copies.

Control Probes: The standard control probe for ddPCR is targeted to RPP30, a non-CNV housekeeping gene. However, since amylase is in a late replicating region ${ }^{161}$, DNA isolated from replicating cells will naturally have less of it than other parts of the genome - the parts that have already replicated. To counteract this, we used a probe assay targeted to just outside the amylase region, called Near_AMY.

Genotype Calling: The output of the droplet reader is a scatterplot with FAM fluorescence on the $\mathrm{y}$-axis and HEX/VIC on the $\mathrm{x}$ axis. Each dot represents a droplet. The Quantasoft software draws suggested thresholds for positive FAM and HEX droplets. The experimenter checks those thresholds and redraws if needed (while still blinded to sample identity). A CNV copy number call is found by dividing the number of droplets fluorescing FAM, corresponding to the CNV target, by the number of droplets fluorescing VIC, the control target (both numbers being Poisson corrected). Before the 
final genotype call, the raw CNV calls of each plate are corrected by a plate-wide correction factor, generally between 0.97-1.05.

HapMap Samples: Plates containing the HapMap ${ }^{158}$ DNA samples for CEU and YRI were subjected to ddPCR in three reactions for $A M Y 2 B, A M Y 2 A$, and $A M Y 1$ using the "assay1" assays listed in Table B-1. All except half the CEU individuals on the $A M Y 2 B$ run were done using the control assay Near_AMY. DNA inputs varied, due to the variation of DNA concentration across the plate, but the ideal DNA concentration strived for was $1 \mathrm{ng} / \mathrm{ul}$ for $A M Y 1$ reactions and $0.5 \mathrm{ng} / \mu \mathrm{l}$ for $A M Y 2 B$ and $A M Y 2 A$ reactions. All copy numbers reported are from a single reaction for each gene. However, we do have multiple runs on file for these assays and others, runs that were done during the optimization stage. 


\section{Table B-1}

a.

ddPCR Assays:

$\begin{array}{lllll} & \text { Primer } & \text { Primer } & \text { Probe } & \text { Annealing } \\ \text { temperature used }\end{array}$

Controls:

\begin{tabular}{llll}
\hline & GATTTGGACCTGCGAGC & & \\
RPP30 & G & GCGGCTGTCTCCACAAGT & CTGACCTGAAGGCTCT \\
\hline & AAATTTATTGGAGGGAT & TTCAAGTTTGACTGCTAAC & TGGAATAAAGAATCATTGG \\
Near_AMY & GTTGG & TCCTG & GCACAGGT
\end{tabular}

b.

Read-depth bins

\begin{tabular}{|c|c|c|c|}
\hline (hg19): & $\begin{array}{l}\text { Bin } 1 \\
\text { chr1 } 104103087- \\
104137966\end{array}$ & Bin 2 & $\operatorname{Bin} 3$ \\
\hline & chr1 104153652- & & \\
\hline AMY2A - first part & 104161927 & NA & NA \\
\hline $\begin{array}{l}\text { AMY2A - } \\
\text { pseudogene }\end{array}$ & $\begin{array}{l}\text { chr1 104161928- } \\
104210498\end{array}$ & $\begin{array}{l}\text { chr1 104256097- } \\
104304649\end{array}$ & NA \\
\hline AMY1 & $\begin{array}{l}\text { chr1 104161928- } \\
104210498\end{array}$ & $\begin{array}{l}\text { chr1 104210499- } \\
104256096\end{array}$ & $\begin{array}{l}\text { chr1 104256097- } \\
104304649\end{array}$ \\
\hline $\begin{array}{l}\text { AMY1, InCHIANTI } \\
\text { cohort }\end{array}$ & $\begin{array}{l}\text { chr1 104181165- } \\
104209159\end{array}$ & $\begin{array}{l}\text { chr1 104228052- } \\
104256049\end{array}$ & $\begin{array}{l}\text { chr1 104275307- } \\
104303297\end{array}$ \\
\hline Intergenic & $\begin{array}{l}\text { chr1 104137967- } \\
104153344\end{array}$ & $\begin{array}{l}\text { chr1 104211074- } \\
104226763\end{array}$ & NA \\
\hline IntergenicA & $\begin{array}{l}\text { chr1 104137967- } \\
104144300\end{array}$ & $\begin{array}{l}\text { chr1 104220425- } \\
104226763\end{array}$ & NA \\
\hline IntergenicB & $\begin{array}{l}\text { chr1 104144301- } \\
104153344\end{array}$ & $\begin{array}{l}\text { chr1 104211074- } \\
104220426\end{array}$ & NA \\
\hline
\end{tabular}

Estonian Samples: The Estonian DNA was aliquoted into 96 well plates, with a random distribution of under- and overweight samples (KS test, $P=0.51)$. The ddPCR runs were done within a three week period in the same lab, using the same machines, with an experimenter blind to the case-control status of the samples - thus reducing the risk of batch effects and biases. Each sample had one genotyping run of each of these 
assays: AMY2B_assay1, AMY2A_assay1, AMY1_assay1, and AMY1_assay2 (with the exception of Plate 1, which did not have an AMY1_assay2 run).

$A M Y 2 B$ : Initially, 28 Estonians had copy number calls less than 2 for $A M Y 2 B(\mathrm{CN}$ of 0 or 1), a call that should be impossible given $A M Y 2 B$ 's copy number distribution. We hypothesized that an Estonian-specific SNP may be interfering with the assay and ran those samples again using AMY2B_assay2. All the samples, except for 3, then had calls consistent with the known $A M Y 2 B$ distribution ( $\mathrm{CN}$ of 2 or 3 ). In the association analysis, the AMY2B_assay2 genotype calls were used for these samples.

AMY1: Two different assays targeting $A M Y 1$ were used in order to reduce the noise a single assay may have. Two different DNA input concentrations were used with the $A M Y 1$ assays to ensure that each sample had at least one genotype call acquired when it was within the optimal concentration range for ddPCR. In the concentrated reaction, each sample of the plate was pre-calculated to have $>0.2 \mathrm{ng} / \mathrm{ul}$ DNA input concentration at a fixed input volume and were genotyped with the AMY1_assayl. However, given the wide distribution of sample concentrations on each plate, the concentrated run resulted in many of the samples oversaturating their reactions. In the diluted run (AMY1_assay2), each sample input was pre-calculated to produce $>10 \%$ probe-negative droplets, thus diluting the previously over-saturated samples.

$A M Y 2 A$-adjusted averaging of the $A M Y 1$ copy number calls: To avoid biases that may arise from sample DNA concentration differences between cases and controls, we did not filter or clean the data based on concentration and used every genotype call the BioRad QuantaSoft software provided. However, a straight average of these two AMY1 replicate genotype calls was not ideal, since many of the samples had one genotype call 
obtained when the sample was too dilute or over-concentrated, thus adding noise to their better genotype call. Instead, an $A M Y 2 A$-adjusted average was used, as described below.

Given that $A M Y 1$ and $A M Y 2 A$ share parity (that is, their copy numbers are either both odd or both even), we can check the correctness of the AMYI copy number call using the $A M Y 2 A$ call. In practice, this means checking each individual's two replicate $A M Y 1$ calls for concordance with their $A M Y 2 A$ call. If both $A M Y 1$ calls were concordant, they were averaged ( $70 \%$ of samples). If only one was concordant, only the concordant $A M Y 1$ genotype was used ( $24 \%$ of samples). If both calls were not concordant, they were averaged ( $6 \%$ of samples). This resulted in better clustering at integers (average deviation from integer 0.152 , compared to straight averaging 0.179 ) despite having nothing to do with either DNA concentration, distance from an integer, or confidence intervals. It should be noted that a BMI association was done separately with all three arrangements $A M Y 2 A$ adjusted average, straight average, and each run separately - all resulting in $P>$ 0.05 .

\section{Read-depth genotyping:}

Algorithm: As a second method for determining the integer copy number of the CNV segments, we used recent versions of the Genome STRiP software ${ }^{156}$ to determine copy number from whole-genome sequencing data. Briefly, for each $\mathrm{CNV}$, the number of unique sequencing reads falling within the target $\mathrm{CNV}$ were counted for each individual and compared to the expected number of reads. We required a minimum mapping quality of 10 and that the reads were aligned to a unique position on the reference genome, except in cases where the target $\mathrm{CNV}$ is duplicated in the reference genome (such as 
AMY1). The expected number of reads per copy was estimated based on the genome-wide sequencing coverage for each individual, correcting for the alignability of the $\mathrm{CNV}$ segment and for sequencing bias due to GC content. Alignability was estimated by mapping overlapping k-mers from the reference genome back to the reference. For the HapMap cohort (from 1000 Genomes Phase 1), we used a k-mer length of 36 and for the GoT2D, InCHIANTI, and GPC cohorts (which have longer reads), we used a k-mer length of 101. GC-bias was estimated by counting the number of aligned reads in overlapping 400bp windows binned by GC fraction compared to a set of selected reference windows having no evidence of copy number variability.

The vectors of observed and expected read counts were fitted to a constrained Gaussian mixture model with two parameters ( $\mathrm{m} 1$ and $\mathrm{m} 2)$ and a site-specific number of genotype classes corresponding to the potential copy numbers. The number of copynumber classes was based on the individual with the highest observed to expected read count ratio (rounding up to the nearest integer and adding one extra copy number class). The means of each genotype class were constrained to be proportional $(\mathrm{m} 1)$ to the copy number and the variances were constrained to be proportional (m2) to the copy number (or to a small constant $\mathrm{k}=0.2$ for the copy number zero class). After using an expectation maximization (EM) algorithm to determine the most likely values for $\mathrm{m} 1, \mathrm{~m} 2$ and the proportional weighting of each copy-number class, the relative likelihood of the observed read depth given each potential genotype class was calculated for each individual. Fractional copy-number estimates for each individual used in plotting are computed as the observed to expected ratios scaled by $\mathrm{m} 1$. 


\section{Determining the locations and boundaries of the copy-number variable}

genomic segments: We created an initial map of the potentially copy number variable segments at the amylase locus based on the paralogous gene annotations from the reference genome, annotated segmental duplications, and results from previous studies (Groot et al 1989, Perry et al 2007). ddPCR measurements were used to confirm copy number variability at specific primer amplification sites, while measurements from sequencing read depth were used to determined variability (or lack thereof) by interrogating the average copy number per individual across longer genomic segments.

Segmentation was further guided by building an alignability map of the locus by aligning $\mathrm{k}$-mers $(\mathrm{k}=36, \mathrm{k}=70$ and $\mathrm{k}=100)$ from the reference genome back to the reference genome using BWA ${ }^{224}$ and using this alignability map to generate hypotheses about the extent of the copy-number variable segments. The segment boundaries were refined based on prospective genotyping of multiple candidate segments using sequencing read depth and Genome STRiP and then optimizing for segments that yielded integer copy numbers in all samples and high posterior genotype likelihoods (similar to the automated method used in recent versions Genome STRiP for optimizing boundaries in non-repetitive sequence). When some individuals were observed to cluster at midinteger copy number estimates, suggesting the presence of additional copy-number variable sub-segments, we applied this procedure recursively down to the length scale resolvable from the available sequencing data sets. The variability of all segments, except for the intergenic region, was confirmed by designing ddPCR assays to these segments and carrying out ddPCR experiments to confirm the sequencing-based results 
The bins used for the read-depth analysis are in Table B-1. Even though the bins for $A M Y 1$ are substantially larger than the $A M Y 1$ repeated segment, most of the signal Genome STRiP used to call genotypes arose from the $A M Y 1$ repeated segment.

\section{Genotyping InCHIANTI cohort with alternative read-depth method:}

We analyzed 657 samples after quality control checks. Average depth was 7x. We aligned the reads to a repeat masked GRC build 37 reference genome using the mrsFAST ultra version 3.3.1 algorithm, which can align single reads to multiple positions in the genome and so is optimal for regions of variable copy number ${ }^{225}$. Repeats were detected and masked using both RepeatMasker Open-3.0 226 and Tandem Repeats Finder $4.07 b^{227}$. Reads were mapped in single-read mapping mode with a hamming distance threshold of $<4$ bp. We derived GC corrected absolute copy number in 100bp windows using mrCaNaVaR version $0.51^{228}$, a program that predicts from read depth and GC enrichment an absolute copy number. We calculated a mean copy number value for the three combined $A M Y 1$ regions, $A M Y 2 A$ and $A M Y 2 B$.

\section{BioNano Genomics, optical mapping:}

Optical mapping experiments were performed by BioNano Genomics. In brief, optical mapping can be thought of as next-generation restriction mapping. Strands of whole, labeled DNA (nick labeled and backbone stained with YoYo1) are electrophoresed through a NanoChannel array. The array straightens the DNA and the fluorescence left at the nick sites creates patterns that can be used to assemble a whole genome, or pieces thereof, in a manner similar to restriction mapping. Each amylase gene 
has its own restriction pattern, so we can determine the order and orientation of the genes from these patterns.Long, whole strands of DNA $(\sim 300 \mathrm{~kb})$ are spread on a slide. A restriction enzyme nicks the DNA, and the nicks are labeled with a fluorophore. The strands are photographed, and assemblies are built by aligning the patterns of nicks to a reference genome. De novo assembly is also possible. A mix of de novo and referenceassembling was used for this project.

\section{Clustering of SNP haplotypes (spiderplot):}

All unrelated individuals in the 1000 Genomes European populations (CEU, TSI, GBR, FIN, IBS ${ }^{207}$ that had amylase genotypes where the two structural haplotypes could be unambiguously determined (phased) were selected for SNP clustering in the spiderplot. These individuals' amylase haplotypes and SNP genotypes (downloaded from the 1000 Genomes website, Omni chip data, (ftp://ftp.1000genomes.ebi.ac.uk/vol1/ftp/ technical/working /20120131_omni_genotypes_and_intensities/) were combined in a .bgl file and phased as a group using BEAGLE version 3.3.2 $2^{229}$ under the default conditions with no reference panel. The spiderplot created was from the 23 closest SNPs with a minor allele frequency greater than $1 \%$ and were outside of the variable region (resulting in 9 SNPs upstream, 14 downstream). The spiderplot was created by traversing the set of SNP haplotypes in both directions from the target variant (amylase) and grouping the haplotypes according to their state at each successive SNP to form two tree structures representing the left and right flanks. At each split, the branch corresponding to the minor allele was plotted above the branch corresponding to the major allele. The color of each 
horizontal segment indicates the allele frequency of the next SNP on the branch and the thickness corresponds to the number of haplotypes sharing that segment.

\section{Association of SNPs to haplotypes and imputation:}

The individuals of the GPC cohort that had genotypes where the two amylase haplotypes could be unambiguously determined were used to search for tag SNPs for each haplotype. SNPs under 1\% minor allele frequency and those within the copy number variable region were not used. Every remaining SNP was correlated with every haplotype, in turn, using a pearson test. During the test, all amylase haplotypes were recoded as 0 or 1 , with the target haplotype being $1 . P$ values were permuted by shuffling the amylase haplotypes 1 million times to create a distribution of possible $r^{2}$ values for each SNP.

The efficiency of imputation was calculated based on leave-one-out trials. Briefly, each individual's amylase haplotypes were masked, in turn, within the unphased data and

phased using BEAGLE ${ }^{229}$ under default conditions using no reference panel. The amylase haplotypes that BEAGLE assigned were extracted and compared to the true values of the masked individuals. R-squared values were obtained with a pearson correlation and $P$ values were calculated from 1 million permutations, creating an $r^{2}$ distribution.

\section{Association of SNPs to diploid copy number of the amylase genes:}

Separately, the GPC cohort, the GoT2D cohort, and the Europeans of 1000 Genomes were genotyped with read-depth analysis, and the diploid copy number calls were combined with the individuals' SNP genotypes (recoded as 0,1 , or 2 for the number 
of alternative alleles present). Linear regression using each SNP genotype, in turn, as the predictor for $A M Y 1$ diploid copy number gave the effect size (slope of the line/coefficient

of the regression) and $r^{2}$ for the association of each SNP. $P$ values were permuted by shuffling the amylase genotypes at least ten times (and up to a million times for the best SNPs) to create a distribution of effect sizes to which we compared the 'true' effect size. The permuted $P$ values, $r^{2}$ values, and effect sizes replicated across cohorts.

\section{Searching GWAS for associated SNPs:}

Given the GPC cohort's greater sample size and larger set of SNPs genotyped, we chose this cohort to display in Figure 4-1. We downloaded the publicly available GIANT consortium (http://www.broadinstitute.org/collaboration/giant) SNP P values for BMI association $^{94}$ and compared each SNP's BMI $P$ value (as determined by GIANT) with its association to $A M Y 1$ copy number (as determined by this study).

\section{SNP Genotyping of Estonians, along with the polygenic score and ancestry}

\section{analysis:}

The Estonian extremes had been previously genotyped with ExomeChip-v1.1 (Illumina Inc). As several replating events occurred between the array and ddPCR genotyping, the samples from the ddPCR batch were further genotyped by using Sequenom's MassARRAY system (which allows a single base extension with allelespecific masses). A multiplex pool of 24 SNPs was used for BMI association in the Estonian cohort with 10 SNPs selected from the AMY locus (the best associated SNPs from 1000 Genomes) and 14 previously identified SNPs associated with BMI ${ }^{94}$ 
(Supplementary Table 10). The latter set of SNPs was assayed to estimate the statistical power in the Estonian cohort to validate BMI linked genetic associations. Genotypes were called by mass spectrometry. Samples with less than an $85 \%$ genotype success rate and SNPs with less than an $85 \%$ genotype success rate and/or poor Hardy-Weinberg $P$ value $(<0.001)$ were excluded from the analysis. 10 AMY locus SNPs and 11 BMI SNPs passed the QC and were used in subsequent analysis. We observed 100\% genotype concordance between the MassARRAY and ExomeChip-v1.1 SNP calls.

Table B-2. SNPs for polygenic score in Estonians

\begin{tabular}{|c|c|c|c|c|c|c|}
\hline $\begin{array}{c}\text { Spelotes et } \\
\text { al }\end{array}$ & SNP & Effect Allele & Beta (SE) & EAF & VarExp & NPC \\
\hline FTO & rs1558902 & A & $\begin{array}{l}0.39 \\
(0.02) \\
\end{array}$ & 0.42 & $0.34 \%$ & 14.03 \\
\hline MC4R & rs6567160 & C & $\begin{array}{l}0.23 \\
(0.03)\end{array}$ & 0.24 & $0.10 \%$ & 4.149 \\
\hline SEC16B & rs543874 & G & $\begin{array}{l}0.22 \\
(0.03) \\
\end{array}$ & 0.19 & $0.07 \%$ & 2.905 \\
\hline FL35779 & rs2112347 & $\mathrm{T}$ & $\begin{array}{l}0.10 \\
(0.02) \\
\end{array}$ & 0.63 & $0.02 \%$ & $\begin{array}{r}0.82 \\
1 \\
\end{array}$ \\
\hline BDNF & $\begin{array}{l}\text { rs11030104 } \\
\text { (rs10767664) }\end{array}$ & A & $\begin{array}{l}0.19 \\
(0.03)\end{array}$ & 0.78 & $0.07 \%$ & 2.855 \\
\hline MTCH2 & rs3817334 & $\mathrm{T}$ & $\begin{array}{l}0.06 \\
(0.02)\end{array}$ & 0.41 & $0.01 \%$ & 0.412 \\
\hline FAIM2 & rs7138803 & $A$ & $\begin{array}{l}0.12 \\
(0.02) \\
\end{array}$ & 0.38 & $0.04 \%$ & 1.65 \\
\hline MAP2K5 & rs16951275 & $\mathrm{T}$ & $\begin{array}{l}0.13 \\
(0.02)\end{array}$ & 0.78 & $0.03 \%$ & 1.228 \\
\hline GPRC5B & $\begin{array}{l}\text { rs12446632 } \\
\text { (rs12444979) }\end{array}$ & G & $\begin{array}{l}0.17 \\
(0.03) \\
\end{array}$ & 0.87 & $0.04 \%$ & 1.628 \\
\hline $\mathrm{SH} 2 \mathrm{~B} 1$ & rs3888190 & $A$ & $\begin{array}{l}0.15 \\
(0.02) \\
\end{array}$ & 0.4 & $0.05 \%$ & 2.062 \\
\hline NEGR1 & rs3101336 & C & $\begin{array}{l}0.13 \\
(0.02)\end{array}$ & 0.61 & $0.04 \%$ & 1.643 \\
\hline & & & & SUM & $0.81 \%$ & 34.02 \\
\hline
\end{tabular}


The software program PLINK ${ }^{230}$--score was used to build a single quantitative index of genetic susceptibility load for obesity. For that, the 11 BMI SNPs' allele dosages were weighted against the effect-sizes reported in Speliotes et al. and added up to a single polygenic score. Based on the estimated total trait variation explained reported in Speliotes et al., the constructed polygenic score is capturing roughly $0.8 \%$ of BMI variation.

ExomeChip data was also used to account for potential population stratification in the extremes sample. ExomeChip genotype data was quality controlled using PLINK ${ }^{230}$ and standard quality control parameters -1 ) sample call-rate $>95 \% ; 2$ ) marker call-rate $>95 \%$; 3) marker allele frequency $>1 \%$ and 4) Hardy-Weinberg $P$ value $<1 \times 10^{-6}$. Cleaned data was combined with HapMap2 genotypes (downloaded from the PLINK resources page) and subsequently analyzed for population structure using the multidimensional scaling (MDS) function in PLINK. Resulting MDS plots show that, although Estonian samples cluster tightly with the CEU cluster, slight structuring is present within the cohort. For that reason, three first MDS vectors were used as covariates in the subsequent association analysis.

\section{Phenotype normalization in the Estonian and GoT2D cohort:}

The standard GIANT Consortium protocol for normalizing the measures-ofobesity phenotype normally consists of adjusting BMI scores in a gender-stratified way for age, age-squared and genetic ancestry vectors (usually three and obtained through PCA or MDS analysis of genome-wide genotype) by fitting a linear regression model. 
Next, the residuals from the model are transformed using an inverse normal transformation and used in subsequent association analyses.

This protocol was slightly modified for normalizing the 1,000 Estonians to account for the extreme-design and to more precisely capture the underlying trait distribution in the whole sampling cohort of $\sim 51,000$. First, the previously described trait normalization (except only age and age-square were used as covariates) was separately performed in females $(\mathrm{N}: 32,724)$ and males $(\mathrm{N}: 17,352)$, resulting in normalized BMI statistics for the 1,000 extreme samples. In subsequent analyses, both genders were analyzed together and sex and three MDS genetic vectors (estimated using ExomeChip data) were used as covariates to account for both gender differences and population stratification.

\section{Power Analysis Estonian Cohort:}

In order to make sure that the Estonian Extremes design has sufficient statistical power to find the associations reported in Falchi et al., we used the Genetic Power Calculator (at http://pngu.mgh.harvard.edu/ purcell/gpc/) ${ }^{231}$, since it has the option to account for threshold-selected quantitative trait design. Falchi et al. reports that $A M Y 1$ copy number explains $0.66 \%$ to $4.40 \%(95 \% \mathrm{CI})$ of the genetic variance in BMI. By using the same calculations as Falchi et al used to estimate the total variance explained for obesity, we back-calculated the mean total BMI variance explained and estimated it as $1.11 \%(95 \% \mathrm{CI}=0.461-1.79)$. The phenotypic thresholds in standard deviation units for defining the case and control sets were obtained from the normalized BMI scores 
described in the previous paragraph and were as follows in standard deviation units -1) Case thresholds +2.0 and +4.0 ; 2) Control thresholds -1.2 and -4.2 .

The genetic effect sizes, trait-increasing allele frequencies, and respective total trait variation explained for the SNPs were obtained from the latest GIANT Consortium full report ${ }^{16}$. For the GIANT Consortium based power analysis, the stage 1 sample size of 124,000 was used. For estimating our power with the polygenic-scores, all individual SNP-based total trait variation explained were summed into one estimate $(0.81 \%)$ and a conservative trait increasing allele frequency of $10 \%$ was used.

\section{Association analysis in the Estonian sample cohorts:}

Both logistic and linear regression models were used to detect association between BMI and the 21 directly genotyped SNPs using PLINK ${ }^{230}$. In the linear regression model, the normalized BMI scores were used, and in both models, gender and three genetic vectors were used as covariates. We used the integer genotypes of the copy number of all three amylase genes obtained through ddPCR in the same 1,000 individuals. For the $A M Y 1$ gene, four different copy number estimates were used $A M Y 2 A$ adjusted average, straight average, and both genotyping runs separately (as described in section AMY2A-adjusted averaging). Again both logistic and linear regression models were fitted using the same phenotype and covariates in $\mathrm{R}^{232}$. A similar analytical framework was used to detect the association between BMI and the constructed polygenic score. No association between the AMY genes' copy number and obesity was observed by either model. 


\section{Association analysis in the InCHIANTI cohort:}

We regressed the copy number values against body mass index corrected for age and sex. We used all copy number calls regardless of quality. Our analyses did not provide any evidence of an association between amylase copy number and BMI in $A M Y 1$ $(P=0.53), A M Y 2 A(P=0.37)$ and $A M Y 2 B(P=0.49)$. Using the more refined method of classifying $A M Y 1$ copy number using the triplicated regions of $A M Y 1$ alone, we still did not see any association with BMI $(P=0.50)$.

\section{Association analysis in the GOT2D cohort:}

The dataset was first divided into T2D-cases $(\mathrm{N}: 1,437)$ and controls $(\mathrm{N}: 1.374)$. The BMI phenotype was transformed using the GIANT protocol (described above adjusting BMI scores in a gender-stratified way for age and age-squared by fitting a linear regression model and subsequently applying inverse normal transformation on resulting residuals from the model). Cases and controls were analyzed separately and the copy number values were regressed against normalized BMI scores while adjusting for gender and source-cohort. All copy number calls were used, regardless of quality. The latter variable was included in order to correct for population stratification as the GOT2D sample consists of 9 separate cohort-collections (Botnia, Diabetes-Registry, FUSION, Helsinki, KORA, Malmö, MPP, STT and WTCCC). Whereas both sub-cohorts (cases and controls) had $>95 \%$ power to replicate the Falchi et al result (total variance explained $1.11 \%$ ), we did not observe nominally significant associations with any of the AMY locus copy numbers in either sub-cohort. 
To test for BMI-dependent effects of amylase that could have been masked in this relatively high BMI cohort, we also tested the tails of the distributions for association.

\begin{tabular}{|c|c|c|c|c|}
\hline \multicolumn{5}{|l|}{ Controls } \\
\hline $\begin{array}{l}\text { Individuals selected for } \\
\text { association }\end{array}$ & $\begin{array}{l}\text { Sample } \\
\text { Size }\end{array}$ & $\begin{array}{l}P \\
\text { value }\end{array}$ & Odds ratio & Beta \\
\hline Normal BMI (18.5-24.9) & 175 & 0.3 & & $\begin{array}{l}0.01(-0.018- \\
0.038)\end{array}$ \\
\hline Non-obese (<30 BMI) & 620 & 0.11 & & $\begin{array}{l}0.015(-0.005- \\
0.035)\end{array}$ \\
\hline $\begin{array}{l}10 \% \text { tails (the most vs. least } \\
\text { obese) }\end{array}$ & 274 & 0.53 & $\begin{array}{l}1.046(0.908- \\
1.204)\end{array}$ & \\
\hline $20 \%$ tails & 550 & 0.75 & $\begin{array}{l}1.017(0.919- \\
1.124)\end{array}$ & \\
\hline $30 \%$ tails & 824 & 0.15 & $\begin{array}{l}1.061(0.980- \\
1.148)\end{array}$ & \\
\hline \multicolumn{5}{|l|}{ Cases } \\
\hline $\begin{array}{l}\text { Individuals selected for } \\
\text { association }\end{array}$ & $\begin{array}{l}\text { Sample } \\
\text { Size }\end{array}$ & $\begin{array}{l}\mathrm{P} \\
\text { value }\end{array}$ & Odds ratio & Beta \\
\hline Normal BMI (18.5-24.9) & 489 & 0.03 & & $\begin{array}{l}0.021(0.019- \\
0.023)\end{array}$ \\
\hline Non-obese (<30 BMI) & 1092 & 0.75 & & $\begin{array}{l}0.002(-0.002- \\
0.006)\end{array}$ \\
\hline $\begin{array}{l}10 \% \text { tails (the most vs. least } \\
\text { obese) }\end{array}$ & 288 & 0.29 & $\begin{array}{l}1.162(0.882- \\
1.531)\end{array}$ & \\
\hline $20 \%$ tails & 574 & 0.36 & $\begin{array}{l}1.052(0.944- \\
1.173)\end{array}$ & \\
\hline $30 \%$ tails & 862 & 0.54 & $\begin{array}{l}1.026(0.945- \\
1.115)\end{array}$ & \\
\hline
\end{tabular}




\section{Het-Seq}

\section{EXPERIMENTAL PROCEDURES}

\section{Isolation of Transcriptome Compartments}

Nascent and early transcripts were isolated using described methods ${ }^{209,233-235}$.

Briefly, lymphoblastoid cells were pulse-labeled with $200 \mu \mathrm{M}$ of 4-thiouridine (4SU) for 5 and 60 minutes, respectively, with DMSO as a negative control. RNA was extracted using Trizol (Invitrogen), purified with RNeasy columns (Qiagen), and eluted to a concentration $>0.4 \mathrm{ng} / \mu 1$. Purified RNA was denatured, and the $4 \mathrm{SU}$-incorporated sites were biotinylated with $1 \mathrm{mg} / \mathrm{ml}$ EZ-link biotin-HPDP by incubation at $65^{\circ} \mathrm{C}$ for 1.5 hours, then at $25^{\circ} \mathrm{C}$ for an additional 1.5 hours. Unincorporated biotin-HPDP was removed by two washes with chloroform-isoamyl alcohol (24:1) and centrifugation in phase-lock-gel tubes (Eppendorf). Biotinylated RNA was captured and purified using MyOne streptavidin $\mathrm{C} 1$ beads (Invitrogen) before elution in 5\% $\beta$-mercaptoethanol. RNA-seq libraries were then constructed using TruSeq library preparations (Illumina). Nuclear and cytoplasmic fractions were harvested from $20 \times 10^{6}$ lymphoblastoid cells by dounce homogenization and centrifugation as described ${ }^{236}$. In short, harvested cells were washed in cold phosphate buffered saline (PBS) before washing in cold hypotonic buffer (20 mM K-HEPES pH 7.8, $5 \mathrm{mM}$ potassium acetate, $1.5 \mathrm{mM}$ magnesium chloride, $1 \mathrm{mM}$ dithiothreitol, $1 \mathrm{mM} \mathrm{PMSF}$, and $50 \mathrm{U} / \mathrm{ml}$ RNase inhibitor). Cells were then resuspended in $0.25 \mathrm{ml}$ of cold hypotonic buffer and dounce homogenized (roughly 20 strokes or until $>90 \%$ of cells were fragmented as assessed by Trypan blue staining, which marks dead cells) on ice. Lysates were centrifuged to pellet the intact nuclei, and RNA was extracted using Trizol to yield the nuclear fraction. The 
cytoplasmic fraction in the supernatant was supplemented with $400 \mathrm{mM}$ potassium acetate, $20 \mathrm{mM}$ potassium HEPES, $15 \mathrm{mM}$ magnesium acetate, and $0.5 \%$ deoxycholate. Lysates were clarified and extracted prior to RNA-seq library construction.

Polyribosomes were isolated using methods previously described ${ }^{210}$. Briefly, $20 \times 10^{6}$ cells were exposed to $0.1 \mathrm{mg} / \mathrm{ml}$ cycloheximide (or puromycin as a control) for 45 minutes, then washed, homogenized, and centrifuged as described for the cytoplasmic fraction except the hypotonic buffer was supplemented with either $0.1 \mathrm{mg} / \mathrm{ml}$ cycloheximide or puromycin. The supernatant was layered on top of $11.2 \mathrm{ml}$ linear $15-$ $50 \%$ sucrose gradients, which were centrifuged at $35,000 \mathrm{rpm}$ at $4^{\circ} \mathrm{C}$ in a pre-cooled SW41 rotor. Fractions of $350 \mu 1$ were collected for a total of 30-32 fractions per gradient, and polyribosome-containing fractions were identified using a combination of spectroscopy and quantitative RT-PCR to detect GAPDH and SLBP. Polyribosome fractions were pooled, depleted of ribosomal RNA, and subjected to RNA-seq library construction.

Control RNA fractions were created by two methods: total RNA, by ribosomal RNA depletion (Invitrogen); and mRNA, by poly(A) purification followed by TruSeq library construction (Illumina).

\section{Enrichment for heterozygous sites using Het-Seq}

We identified candidate SNPs by selecting those that had at least three reads per half-lane of sequence data in each fraction in each individual as judged by our preliminary RNA-seq data (13,406 in NA19240; 11,402 in NA12878) and excluding those that came from highly expressed genes in LCLs (including Immunoglobulin, $H L A$, 
and $A C T B$ genes). We then designed Het-Seq probes that would flank the hetSNP on both sides, excluding the SNP within a five base pair loop roughly in the center of the 120pb probe (no closer than 30bp from either end). Whenever a hetSNP resided near an annotated splice site, we included additional probes to account for alternative splicing. This was not done by hand, but by an R algorithm. These custom probe sets were ordered from Agilent as in-solution hybridization probes.

\section{Sequencing}

All libraries were molecularly barcoded and sequenced (to a half-lane of coverage) on an Illumina HiSeq2000 using paired-end 75 bp reads.

\section{Alignment pipeline and other analysis details, in brief}

Reads were obtained in FASTQ format from the Illumina HiSeq2000, then adapter-trimmed by Picard's MarkIlluminaAdapters (http://picard.sourceforge.net). They were aligned by BWA using the paired-read method with a read-trim score of 5. They were aligned to a "neutral reference", in which all SNP locations known in that individual were changed to N. In addition, they were aligned to a neutral, junction reference that spanned exon-exon junctions. The genomic alignment reads and junction alignment reads were then simultaneously compared for each read pair, and the most parsimonious alignment was selected. These aligned reads were then combined with the original data using MergeBamAlignment and PCR duplicates were flagged using MarkDuplicates. We normalized GC content bias by empirically measuring GC content for all SNPs in a library, then running a linear regression against the observed SNP alleles to determine a 
correction factor for the binomial test. We filtered out SNPs that aligned nowhere, with low quality, to the wrong location, or multiple locations in an in silico alignment experiment. In addition, SNPs overlapping known pseudogenes and SNPs with $P$ values $<10^{-3}$ in genomic DNA were removed. Details on logic and reference design are in the extended experimental procedures.

\section{Allele-neutral alignment}

The two samples used in this study are GM19240 and GM12878, which have both been deeply sequenced by 1000 Genomes (phase 2), giving us knowledge of the variants present. We used this information to construct a "neutral reference" - one in which all SNP locations are changed to "N". Without a neutral reference genome, reads containing an alternative allele will always have at least one mismatch. This will reduce the number of correctly aligned alternative allele-containing reads, thus biasing the allele count in favor of the reference allele. Setting each allele position to " $\mathrm{N}$ " in the reference genome removes this alignment bias by assigning the same number of mismatches to every allelecontaining read.

\section{Avoidance of double-counting}

Potential PCR duplicates were systematically identified using the Picard package's MarkDuplicates program and removed from analysis. When a single set of paired-end reads made two observations of the same SNP (by sequencing from both ends of the RNA-seq molecule), the two SNP calls were compared. The two reads of the paired end sequencing generally agreed about the allelic state of the SNP, and the allele 
was counted once; if the two read pairs were discordant (rare), then the read was discarded.

\section{Analysis of GC bias}

Construction of sequencing libraries has a well-known "GC content effect" in which inserts with extremely high or low GC content are under-represented. Since reads containing different alleles of the same SNP may have different GC content, there can be a bias in the ascertainment of one allele over another by as much as $5 \%$. For SNPs that have a $\mathrm{C}$ or $\mathrm{G}$ allele, there is a bias towards whichever allele moves the $\mathrm{GC}$ content towards 50\%. As expected, A/T and G/C SNPs do not exhibit this bias. We normalized GC content by empirically measuring it for all SNPs in a library, then running a linear regression against the observed SNP alleles to determine a correction factor. This correction factor was used as the null within the binomial test (i.e. replacing 0.50 with 0.493). It had a very small effect on $P$ values for individual SNPs, but contributed to the calibration of global measurements.

\section{Filtering SNPs in difficult-to-align regions}

Some regions of the genome are inherently more difficult to align, potentially affecting two SNP alleles at different rates and biasing observed allelic ratios. To overcome this problem, we screened SNPs in silico for alignment problems. We extracted 40 bp of sequence 5' and 3' to each SNP and constructed a synthetic FASTQ file with the bases set to be high quality. These reads were then mapped using our mapping pipeline, and the locations of reads compared to their known locations. If sequences containing a 
particular SNP did not map at all, mapped at low quality, mapped to the wrong location, or mapped to multiple locations, then that SNP was removed from further analysis. While all four categories had errors, the majority of the errors were due to SNPs mapping to multiple places on the genome. In addition, SNPs that overlapped known or reported pseudogenes were also removed. After all normalization and filtering methods were applied, a final filter on SNPs with extreme $P$ values $\left(10^{-3}\right)$ in genomic DNA were removed.

\section{Junction Library}

Because we aligned RNA to a genomic reference (and most of the sequencing was on spliced RNA), we also constructed a custom reference - a "junction library"- that spanned exon-exon junctions using sequences from a GenCode GTF file (gencode.v3b.annotation.NCBI36.gtf). Each junction was encoded as $10 \mathrm{bp}$ of "N" sequence followed by the last $50 \mathrm{bp}$ of the first exon attached to the first $50 \mathrm{bp}$ of the second exon, then 10bp of N. Reads were processed as in the genomic alignment with the same parameters.

After alignment with the junction reference, reads were converted back to their locations in genomic coordinates with gaps in their cigar strings to represent where the reads spanned introns. The genomic alignment reads and junction alignment reads were then simultaneously compared for each read pair, and the most parsimonious alignment was selected by comparing the mapping quality of the reads, their edit distance to their respective references, and whether the resulting read pair would be chimeric. This 
method produced results similar to those of the Tophat ${ }^{237}$ alignment strategy, while allowing the inclusion of neutral bases at hetSNP sites for allele-neutral alignment.

\section{Pervasive, inflated $\boldsymbol{P}$ values}

Though Het-Seq capture with custom bioinformatics greatly reduces allelic bias in genomic DNA, visualizing empirical $P$ values against those expected by chance with a QQ-plot reveals that a pervasive inflation remains. This seems to be caused by a small bias in all SNPs, as opposed to a few SNPs of large effect, and the biases can be traced back to both biological and technical reasons.

Though it is often assumed that there are always two copies of a locus within a cell, replicating cells have more copies, which can cause an allelic skew if the replication timing differs between two alleles. A chromosome-wide example would be the $\mathrm{X}$ chromosome $^{238}$. The cells divide soon after replicating the inactive $\mathrm{X}$, but the active $\mathrm{X}$ already has two copies by early S phase, causing a skew towards the active X's alleles. We see this skew in our genomic DNA library with a $P$ value of $6.2 \mathrm{e}^{-13}$ (with all the $\mathrm{X}$ loci, collectively) but the skew disappears when we measure an autosome. Though the $\mathrm{X}$ chromosome is the only known large area of effect, there may be smaller pockets of replication differences affecting our results.

Though HetSeq probes were carefully filtered and designed to prevent allelic bias in capture, it was later found that a small subset of probes bound alleles differentially due to microhomology. As well as forming a five base pair loop containing the SNP, they could shift the loop to invariant sequence by binding one allele of the SNP, causing preferential capture. We found that 750 sites in NA12878 and 1178 sites in NA19240 
contained sequence similarity overlapping a targeted SNP, and they were more likely to be called significant.

Since we are not confident we have found all sources of bias, we instead focused on their impact on RNA allelic ratios. The vast majority of skews in DNA were less than 0.10 away from the 0.50 proportion expected for ref/alt allele, and those that were significant were few in number. In addition, with the fewer reads that RNA libraries contain, many of the small skews disappear with the lack of power. So, even though there is a pervasive bias, it is small in effect and should not affect our analyses.

\section{Beta-binomial Modeling}

We observe an allelic ratio $\frac{a_{i}}{a_{i}+b_{i}}$ for each gene, where $a_{i}$ and $b_{i}$ are the number of paternal and maternal reads, respectively. We think of $a_{i}$ as binomial successes out of $n_{i}=a_{i}+b_{i}$ total reads, with a hidden probability $p_{i}$ of success for gene $i$. The underlying distribution of $p_{i}$ represents a mixture of genes that are homozygous in their active regulatory regions, and therefore have $p_{i} \approx 0.5$, and genes that have SNPs in regulatory regions, where $p_{i}$ may be shifted from 0.5 . We modeled this composite distribution of $p_{i}$ as a mixture of two symmetric $\operatorname{Beta}\left(\alpha_{k}, \alpha_{k}\right)$ distributions $(k=1,2)$ with mixing weights $w_{k} . \alpha_{k}$ was fixed using the best fit $\operatorname{Beta}\left(\alpha_{1}, \alpha_{1}\right)$ to gDNA downsampled to the median of RNA library of interest to model the homozygous genes, and the effect sizes of imbalanced genes are distributed $\operatorname{Beta}\left(\alpha_{2}, \alpha_{2}\right)$. The generative model for expected data is thus: for each $i=1,2, \ldots N$, select $k$ with probability $w_{k}$. Then generate $p_{i} \sim \operatorname{Beta}\left(\alpha_{k}, \alpha_{k}\right), a_{i} \sim \operatorname{Binomial}\left(n_{i}, p_{i}\right)$, and set $b_{i}=n_{i}-a_{i}$. 
We fit $w$ and $a_{2}$ using a standard expectation-maximization (EM) approach:

For observation $\left(a_{i}, b_{i}\right)$ the likelihood under the $k$ th Beta distribution is:

$$
p\left(a_{i} \mid n_{i}, \alpha_{k}\right)=L(k, i)=\frac{B\left(\alpha_{k}+a_{i}, \alpha_{k}+b_{i}\right)}{B\left(\alpha_{k}, \alpha_{k}\right)}
$$

Thus the posterior probability that $k$ was selected is:

$$
\gamma_{i k}=\frac{w_{k} L(k, i)}{\sum_{k} w_{k} L(k, i)}
$$

and the log likelihood is:

$$
\Lambda(k, i)=\log \left(\sum_{k} w_{k} L(k, i)\right)
$$

Further the mean posterior of $\log \left(p_{i}\right)$ if $k$ is selected is:

$$
E\left[\log p_{i} \mid a_{i}, n_{i}, \alpha_{k}\right]=X(k, i)=\psi\left(\alpha_{k}+a_{i}\right)-\psi\left(2 \alpha_{k}+n_{i}\right)
$$

Reestimates are obtained by computing:

$$
\begin{gathered}
X_{k}=\frac{\sum_{i} \gamma_{i k} X(k, i)}{\sum_{i} \gamma_{i k}} \\
N_{k}=\sum_{i} \gamma_{i k}
\end{gathered}
$$

Set new weights:

$$
w_{k}=\frac{N_{k}}{N}
$$

and solve:

$$
X_{k}=\psi\left(\alpha_{k}\right)-\psi\left(2 \alpha_{k}\right)
$$

for $\alpha_{2}$

\section{ddPCR Confirmations}

We selected 24 SNPs for confirmation by ddPCR, with 20 assays passing basic quality control tests for off-target activity. Each assay was composed of two probes (FAM and HEX) that were specific for either allele of the target SNP. We used the 
official Bio-Rad protocol for their QX100 Droplet Digital PCR System. Due to the digital nature of ddPCR, it was possible to count the number of cDNA molecules containing each SNP and to calculate an allelic ratio with $95 \%$ confidence intervals. These assays were performed on the same RNA samples that were sequenced and were judged to agree with Het-Seq when the confidence intervals from both methods overlapped. 


\title{
Appendix C. Protocols for amylase genotyping using ddPCR
}

In the lab:

1. Determine the approximate DNA concentration of the sample.

Restriction Digest:

2. Put together the following reaction:

\author{
$1 \mu 1$ of $10 \times$ CutSmart Buffer (New England Biolabs) \\ $0.5 \mu 1$ of EcoRI (20,000 units/ml, New England Biolabs) \\ 80 ng of DNA
}

Water, bringing the reaction volume up to $10 \mu \mathrm{l}$

3. Incubate at $37^{\circ} \mathrm{C}$ for 1 hour.

4. Add $10 \mu l$ of water

$A M Y 2 B$ genotyping:

5. Prepare the following reaction:

$11 \mu \mathrm{l}$ of $2 \times$ ddPCR SuperMix for Probes (Bio-Rad)

$0.55 \mu 1$ of Near_AMY1 assay (40× concentration, ordered from IDT, 12.5

nMoles probe, $45 \mathrm{nMoles}$ of each primer, resuspended in $1250 \mu \mathrm{l}$ water) 
$0.55 \mu 1$ of AMY2B_assay 1 assay $(40 \times$ concentration, ordered from IDT, $12.5 \mathrm{nMoles}$ probe, $45 \mathrm{nMoles}$ of each primer, resuspended in $1250 \mu \mathrm{l}$ water)

$4 \mu 1$ of digested DNA

$5.9 \mu$ of Water

6. Generate droplets using a QX200 droplet generator (Bio-Rad) or QX200 Auto-DG (Bio-Rad) according to manufacturer directions. Seal the plate with an aluminum slip, such as TempPlate (USA scientific).

7. Amplify in a thermocycler following this protocol: $10 \mathrm{~min}$ at $95^{\circ} \mathrm{C}, 50$ cycles of 30 sec at $94^{\circ} \mathrm{C}$ and $60 \mathrm{~s}$ at $60^{\circ} \mathrm{C}$, then $10 \mathrm{~min}$ at $98^{\circ} \mathrm{C}$.

8. Run the droplets on a QX100 droplet reader (Bio-Rad) according to manufacturer directions.

$A M Y 2 B$ analysis

9. With the whole plate selected in the Quantasoft analysis software (Bio-Rad), draw the thresholds for FAM- and VIC-positive calls. These thresholds will be between the clusters of droplets, and the software will have likely called half the samples itself. Download the .csv file

10. Using the "R scripts" part of this chapter, calculate the average DNA concentration of the PCR reaction (script is for a 96-well plate). 
$A M Y 2 A$ Genotyping

11. If the bulk of samples had a $1 \mathrm{ng} / \mu \mathrm{l}$ DNA concentration in the $A M Y 2 B$ ddPCR reaction, as calculated by the script above, then genotype $A M Y 2 A$ in the same manner as $A M Y 2 B$. Use the AMY2A_assay1 mix (mixed the same way as the AMY2B_assay1) and Near_AMY1 mix. The PCR protocol is as follows: $10 \mathrm{~min}$ at $95^{\circ} \mathrm{C}, 50$ cycles of $30 \mathrm{sec}$ at $94^{\circ} \mathrm{C}$ and $60 \mathrm{~s}$ at $57^{\circ} \mathrm{C}$, then $10 \mathrm{~min}$ at $98^{\circ} \mathrm{C}$.

11a. If the bulk of samples did not have a $1 \mathrm{ng} / \mu \mathrm{l}$ DNA concentration, adjust the digested-DNA input into the $A M Y 2 A$ ddPCR reaction mixture and continue with the $A M Y 2 A$ genotyping described above.

$A M Y 2 A$ Analysis

12. Using the Quantasoft software (Bio-Rad), set the thresholds for FAM- and VICpositive calls, as in the $A M Y 2 B$ analysis. Download the .csv file.

13. Using the "R scripts" part of this chapter, use the concentration slider to evaluate the average DNA concentration of the PCR reaction (script is for a 96-well plate).

$A M Y 1$ First Genotyping Run

14. Use the concentration slider to determine the optimum digested-DNA input for the first $A M Y 1$ run. The goal of this run is to "capture" most of the DNA samples, such that most will be at a concentration higher than $0.5 \mathrm{ng} / \mu \mathrm{l}$. Select an input that will capture nearly all samples on the plate. Then, genotype as described above. Use the 
AMY1_assay1 or 2 mix (mixed the same way as the AMY2B_assay1) and Near_AMY1 mix. The PCR protocol is as follows: $10 \mathrm{~min}$ at $95^{\circ} \mathrm{C}, 50$ cycles of $30 \mathrm{sec}$ at $94^{\circ} \mathrm{C}$ and 60 $\mathrm{s}$ at $58^{\circ} \mathrm{C}$, then $10 \mathrm{~min}$ at $98^{\circ} \mathrm{C}$.

AMY1 First Run Analysis

15. Using the Quantasoft software (Bio-Rad), set the thresholds for FAM- and VICpositive calls, as in the $A M Y 2 B$ analysis. Download the .csv file.

16. Using the "R scripts" part of this chapter, use the percent slider to evaluate the average concentration of double-negative droplets (script is for a 96-well plate).

\section{$A M Y 1$ Second Genotyping Run}

17. Use the percent slider to determine the optimum digested-DNA input for the second $A M Y 1$ run. The goal of this run is for every sample to have more than $10 \%$ double-negative droplets (this is to capture the samples that oversaturated their first run with PCR products). Then, genotype as described above. Use the AMY1_assay1 or 2 mix (mixed the same way as the AMY2B_assay1) and Near_AMY1 mix. The PCR protocol is as follows: $10 \mathrm{~min}$ at $95^{\circ} \mathrm{C}, 50$ cycles of $30 \mathrm{sec}$ at $94^{\circ} \mathrm{C}$ and $60 \mathrm{~s}$ at $58^{\circ} \mathrm{C}$, then $10 \mathrm{~min}$ at $98^{\circ} \mathrm{C}$.

$\underline{\text { R script: }}$

\#Import these functions. Code for running is at very bottom 


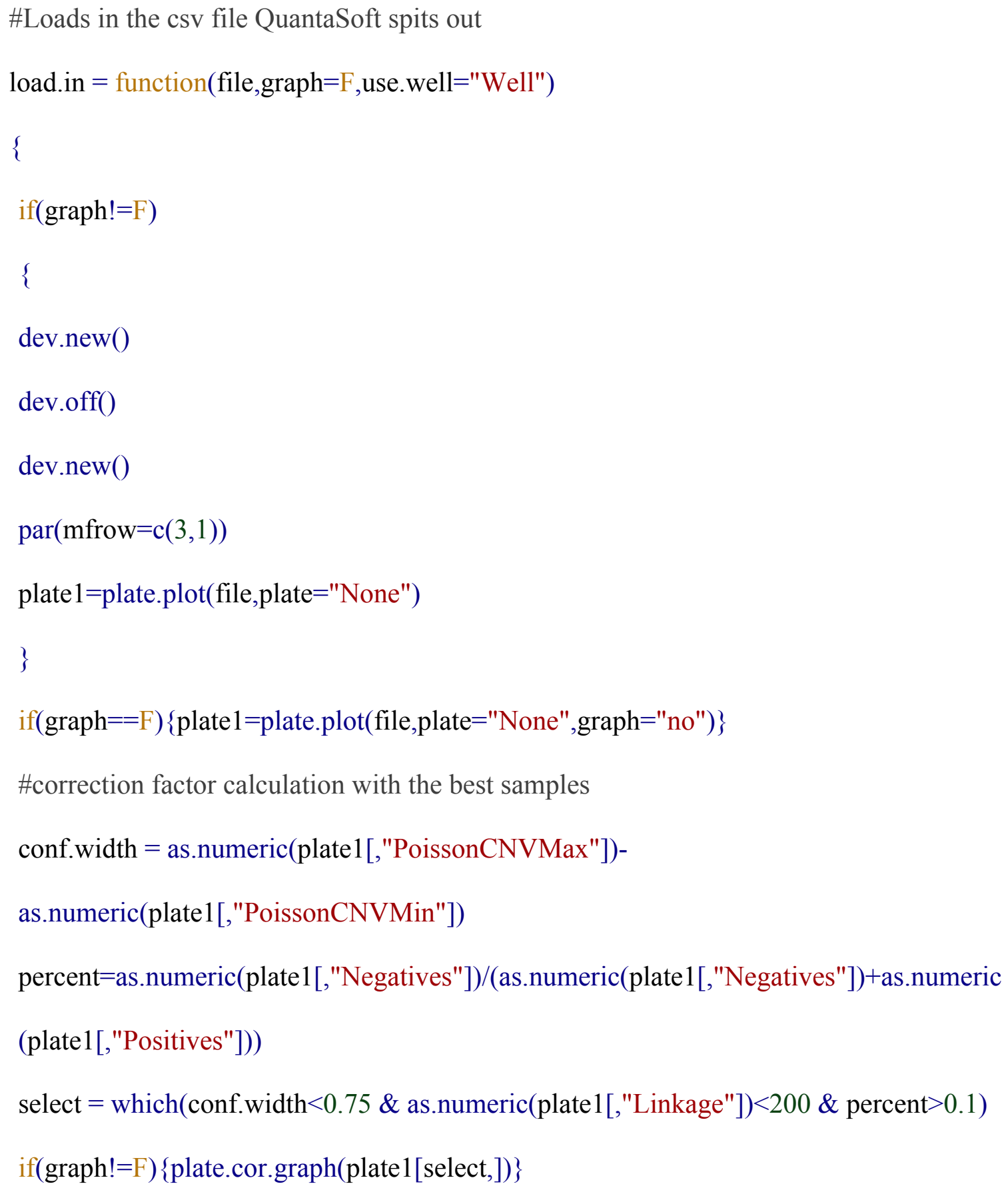


rval=0.001,plot="yes") $\}$

if $($ graph $==\mathrm{F})\{$ corr. amy=plate. $\operatorname{corrected}($ plate1 [select,],file="BMI",min=0.9, $\max =1.2$,int erval=0.001,plot="yes",graph="no") $\}$

plate1[,"CNV"]=as.numeric(plate1[,"CNV"])*corr.amy

plate1[,"PoissonCNVMax"]=as.numeric(plate1[,"PoissonCNVMax"])*corr.amy

plate1[,"PoissonCNVMin"]=as.numeric(plate1[,"PoissonCNVMin"])*corr.amy

conc.ng.ul=(Num(plate1[,"Concentration"])/Num(plate1[,"Ratio"]))/290

conc.ng.ul[is.na(conc.ng.ul) $]=-0.25$

if(graph!=F)

\{

dev.new()

plot(plate1[order(as.numeric(plate1[,"CNV"])),"CNV"],xlab="individual",ylab="copy number",main=paste("Corrected Copy Number \nCorrection Factor

=",corr.amy,"\n",strsplit(file,"/")[[1]][5]),pch=20)

abline $(\mathrm{h}=\mathrm{c}(1: 15), \mathrm{col}=$ "gray")

segments(1:nrow(plate1), as.numeric(plate1[order(as.numeric(plate1[,"CNV"])),"Poisson CNVMax"]),1:nrow(plate1), as.numeric(plate1[order(as.numeric(plate1[,"CNV"])),"Pois sonCNVMin"]),col="dark gray")

$\operatorname{par}(\mathrm{mfrow}=\mathrm{c}(1,1))$

\}

return(cbind(plate1[,c(use.well,"CNV","PoissonCNVMin","PoissonCNVMax","Linkage" )],conc.ng.ul,percent)) 


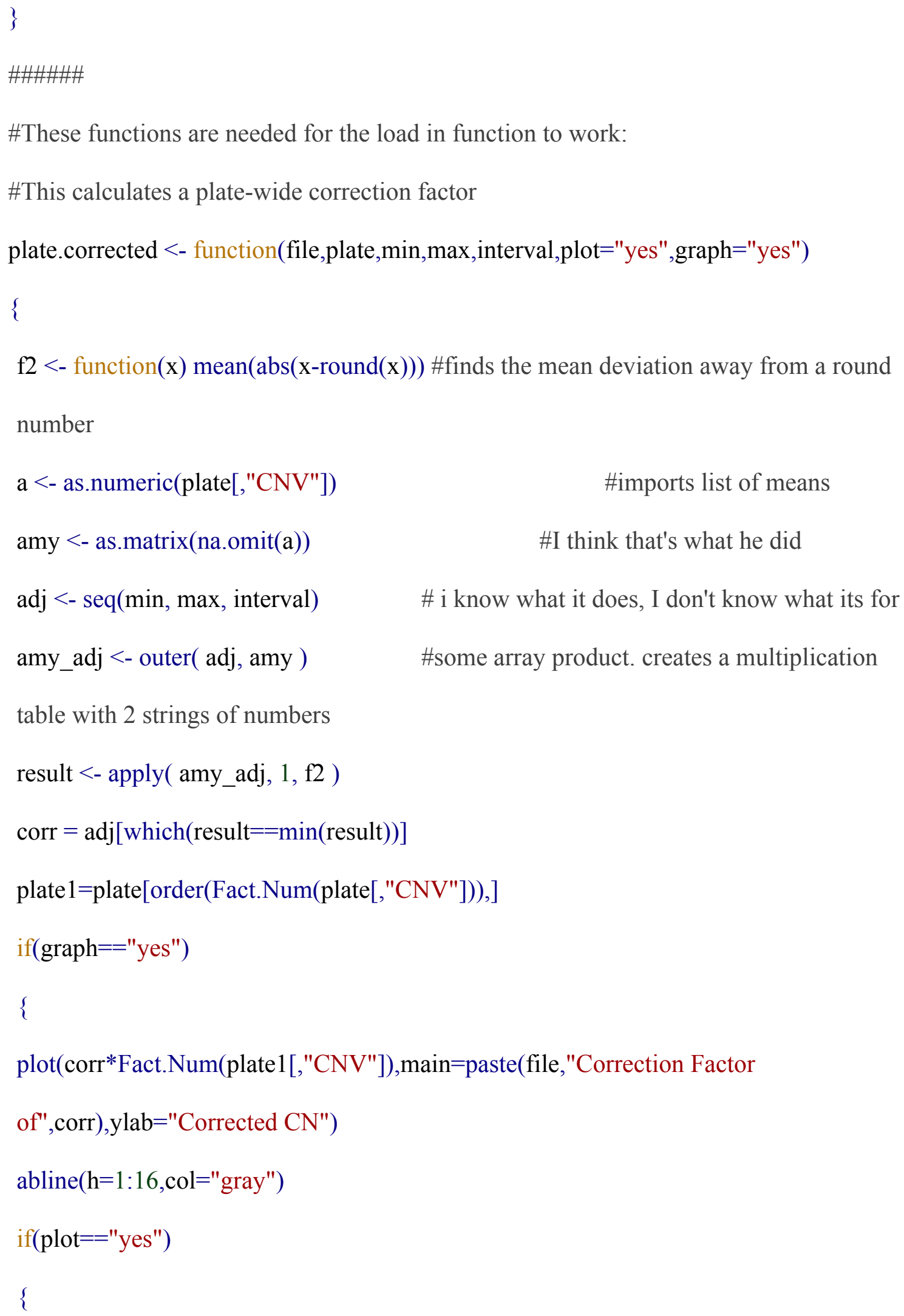




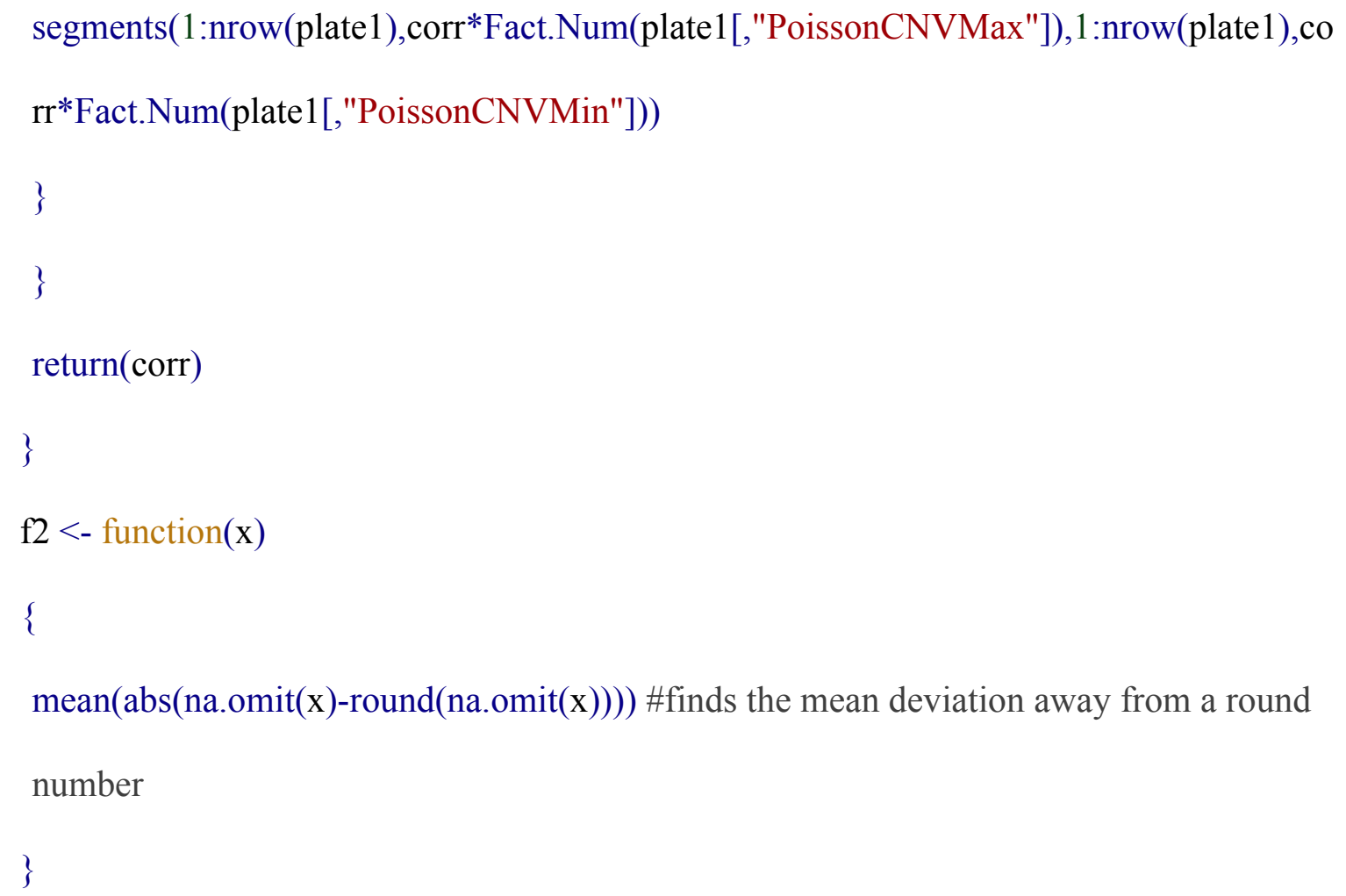


factor",ylab="Mean deviation from integer")\}

\}

Fact.Num $=$ function $(\mathrm{x})\{\operatorname{return}(\operatorname{as} . n u m e r i c(\operatorname{as} . \operatorname{character}(\mathrm{x})))\}$

Num $=$ function $(\mathrm{x})\{$ return $(\operatorname{as} . n u m e r i c(\operatorname{as} . \operatorname{character}(\mathrm{x})))\}$

\#This plots the copy numbers in the csv

plate.plot $<$ - function(file,plate,graph="yes")

\{

Plate1.rep1 $=$ read.csv $($ file $=$ file, header $=\mathrm{F}$, stringsAsFactors $=\mathrm{F})$

colnames(Plate1.rep1) $=$ Plate1.rep1[1,]

Plate1.rep1 = Plate1.rep1[-1,]

plate $1=$ Plate1.rep1[c(which(Plate1.rep1[,5]=="FAMUnknown")),

new.format="no"

$\operatorname{if}($ nrow $($ plate 1$)==0)$

$\{\quad$ plate1 $=$ Plate1.rep1[c(which(Plate1.rep1[,5]=="Ch1Unknown")),

new.format="yes"

\}

rownames $($ plate 1$)=$ plate $1[, 1]$

test.1=plate 1

if(plate!="None")

\{

rownames $($ Platemap $)=$ Platemap[,1]

test.1=merge(plate1, Platemap,all.x=T,by="row.names")[,c(colnames(plate1),plate)]

colnames(test.1)[ncol(test.1)]="Individual" 


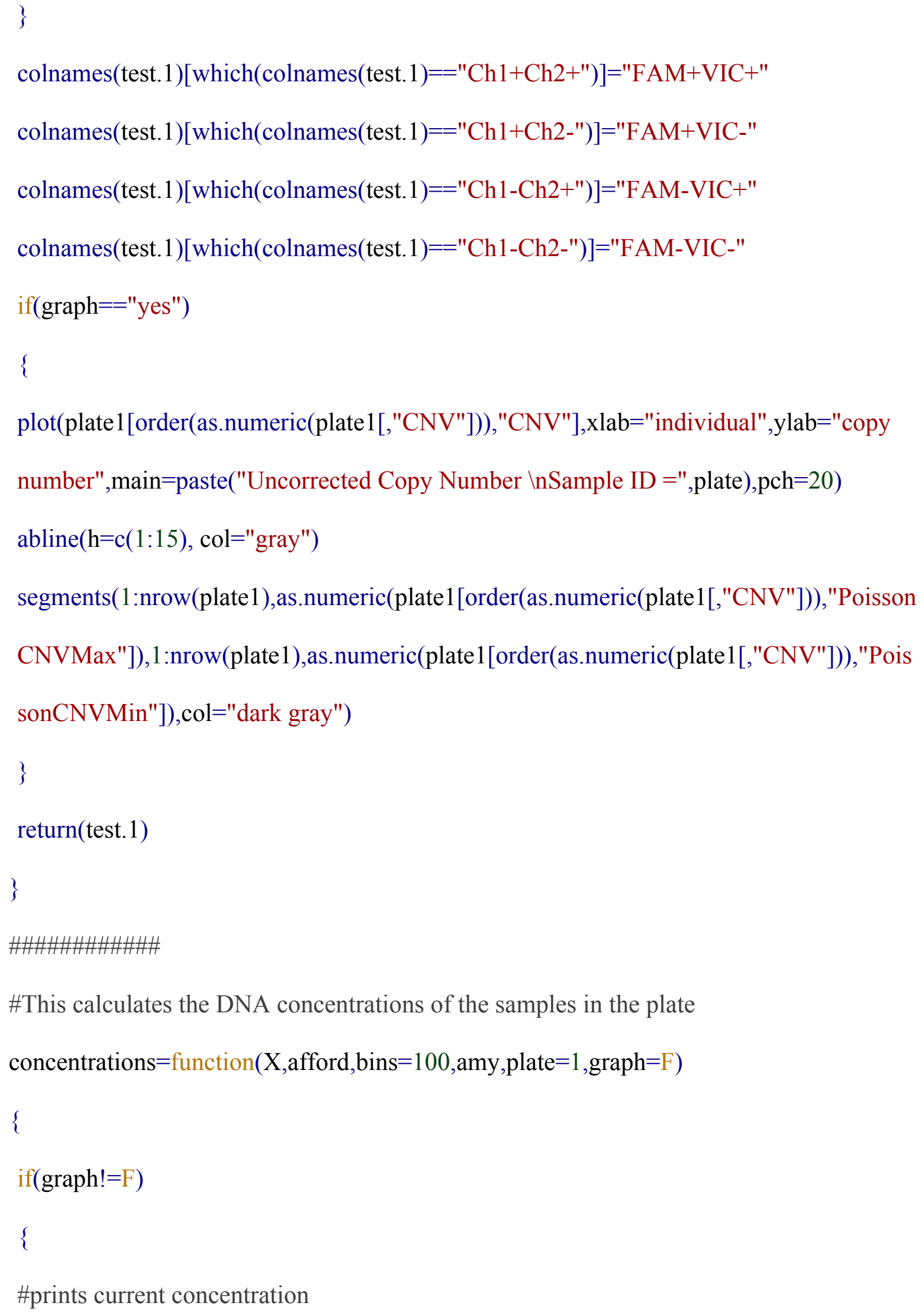




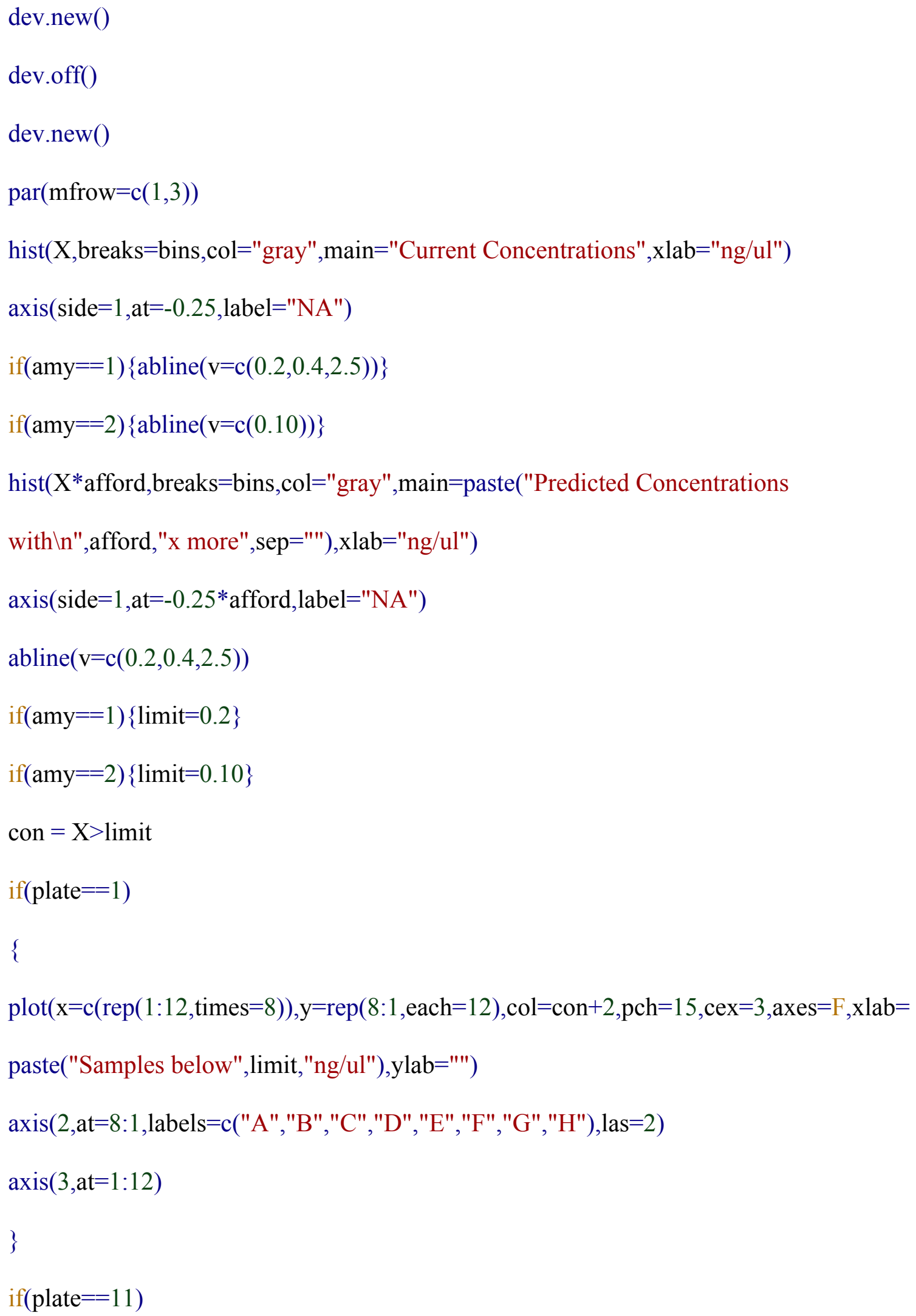


$\operatorname{plot}(\mathrm{x}=\mathrm{c}(\operatorname{rep}(1: 7, \mathrm{times}=8)), \mathrm{y}=\operatorname{rep}(8: 1, \mathrm{each}=7), \mathrm{col}=\mathrm{con}+2, \mathrm{pch}=15, \mathrm{cex}=3, \mathrm{axes}=\mathrm{F}, \mathrm{xla}$ b=paste("Samples below",limit,"ng/ul"),ylab="")

$\operatorname{axis}(2, a t=8: 1$, labels=c("A","B","C","D","E","F","G","H"),las=2)

$\operatorname{axis}(3, \mathrm{at}=1: 7)$

\}

\}

\}

\#This gives you the percentages of negative droplets for each sample. Important for making sure the samples with high AMY1 copy number

\#don't blow out their reactions (ie. have so much AMY1 that there are very few negative droplets)

Percent.slider $=$ function $(\mathrm{x}, \min =0, \max =4$, input, $g r a p h=T)$

\{

dev.new ()

$\operatorname{par}(\mathrm{mfrow}=\mathrm{c}(2,2))$

$\min \cdot \mathrm{per}=(\mathrm{x}[$ which.min $(\mathrm{x}[$, percent"] $)])$,

new.conc $=(\min . p e r[" c o n c . n g . u l "] *-\log (0.1)) /-\log (\min . p e r[, " p e r c e n t "])$

conc.ratio=new.conc/min.per["conc.ng.ul"]

predicted.conc $=$ conc.ratio $*$ Fact.Num $(x[$, "conc.ng.ul"] $)$

predicted.percent $=\exp (\log (x[, " p e r c e n t "]) * a s . n u m e r i c($ conc.ratio $))$

hist(Fact.Num(x[,"conc.ng.ul"])*as.numeric(conc.ratio),main=paste("New concentration 
is ",round(conc.ratio,2),"x of the old oneln Predicted Concentration

Values",sep=""),xlab="Predicted concentrations (ng/ul)",breaks=100,col="gray")

abline $(\mathrm{v}=0.25)$

hist(Fact.Num(x[,"conc.ng.ul"]),main="old concentrations",xlab="concentrations

(ng/ul)",breaks=100,col="gray")

abline $(\mathrm{v}=0.25)$

hist(predicted.percent,main=paste("New concentration is ",round(conc.ratio,2),"x of the old oneln Predicted Concentration Values",sep=""),xlab="Predicted negative

percentage",breaks=100,col="gray")

abline $(\mathrm{v}=0.06)$

$\operatorname{hist}(x[, " p e r c e n t "]$, main="old percentanges",xlab="negative

percentage",breaks=100,col="gray")

abline $(\mathrm{v}=0.06)$

folds $=$ seq $(\min , \max$, by $=0.1)$

captured $=\mathrm{c}()$

kinda $=\mathrm{c}()$

nope $=\mathrm{c}()$

for(i in 1:length(folds))

\{

test=folds[i]*x[,"conc.ng.ul"]

test.ratio=test[1]/x[1,"conc.ng.ul"]

test.predicted.percent=exp $(\log (\mathrm{x}[$,"percent"] $) *$ as.numeric(test.ratio $))$

captured $[\mathrm{i}]=$ length $($ which $($ test $>=0.25 \&$ test.predicted.percent $>=0.10)$ ) 


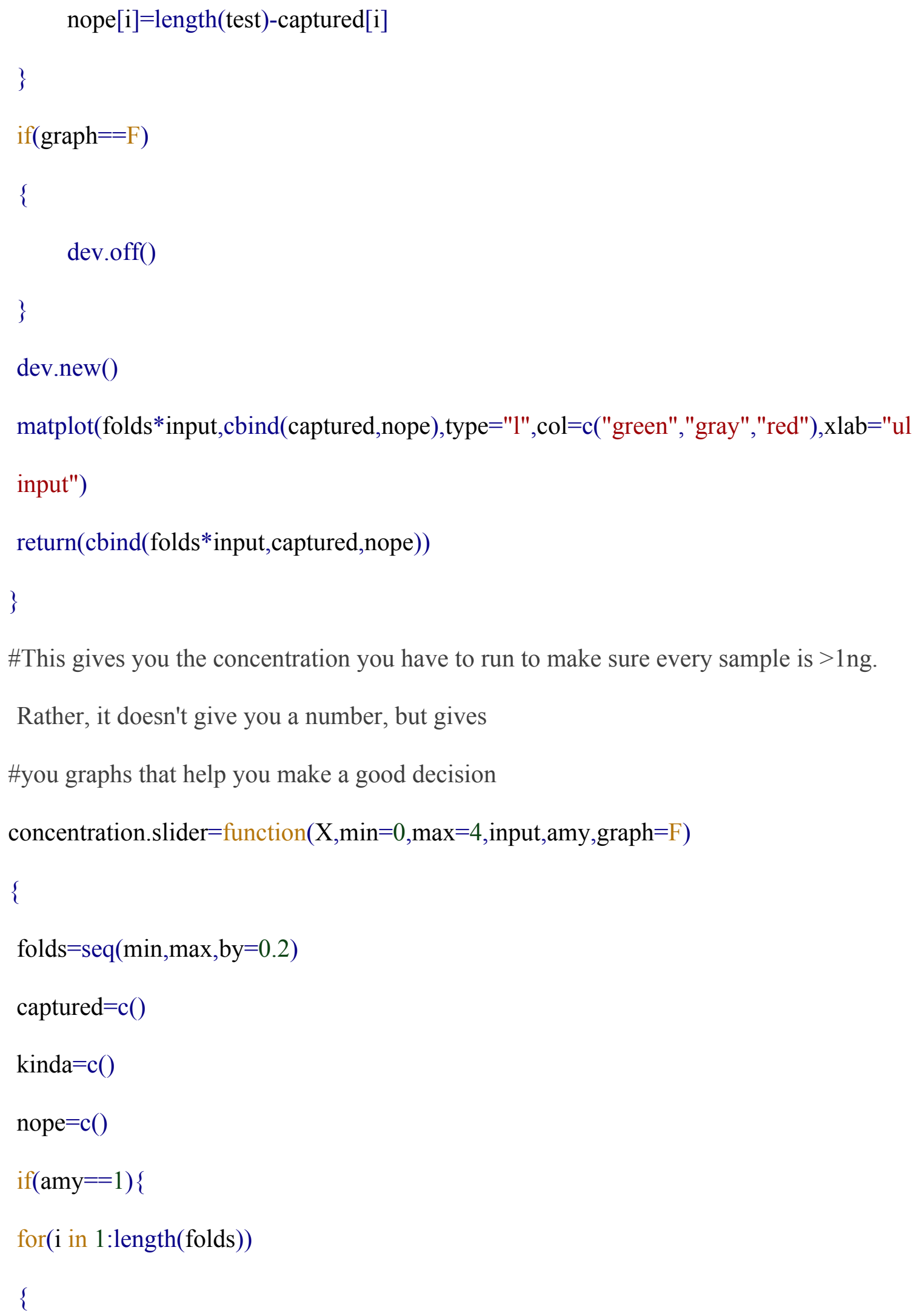




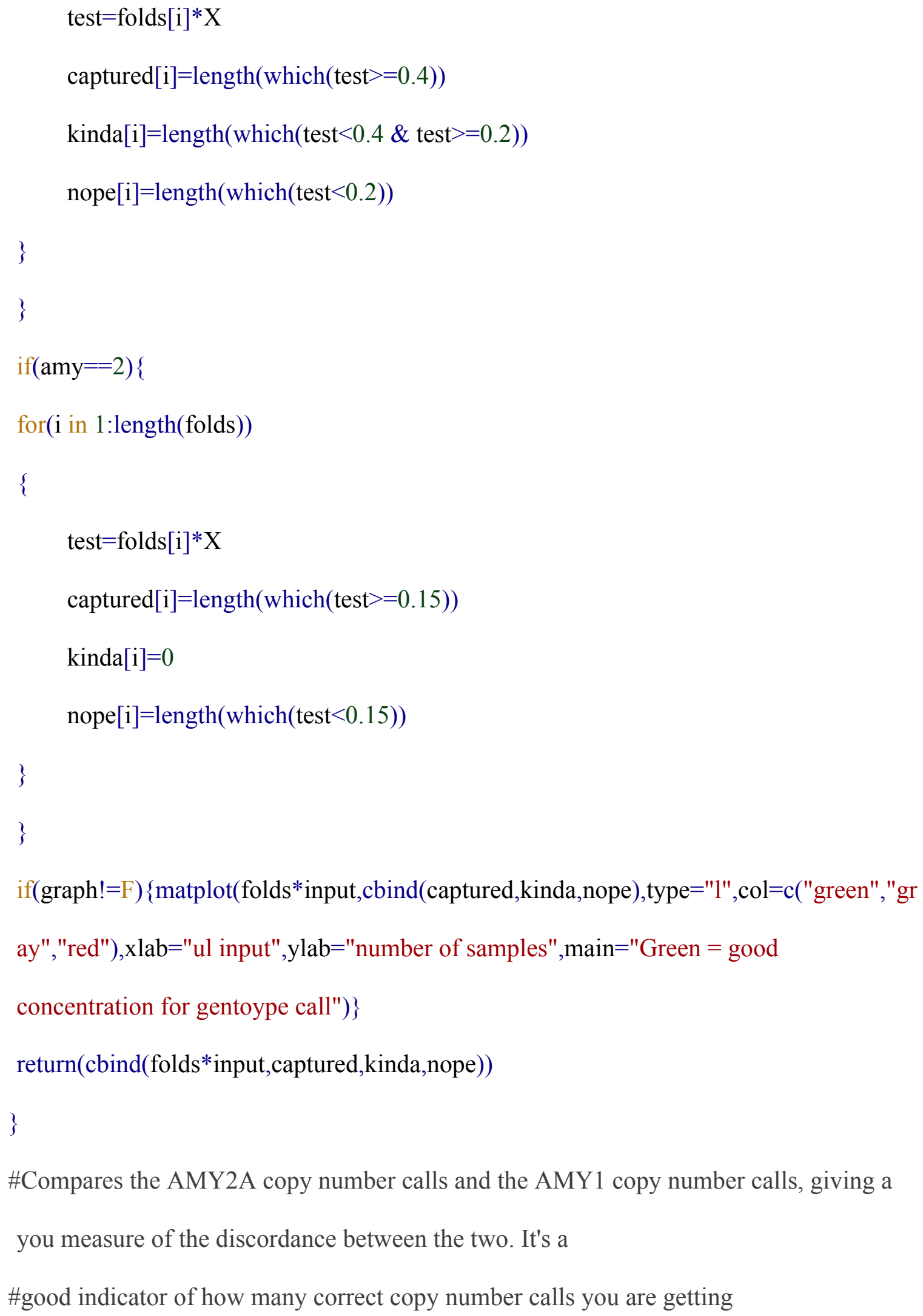




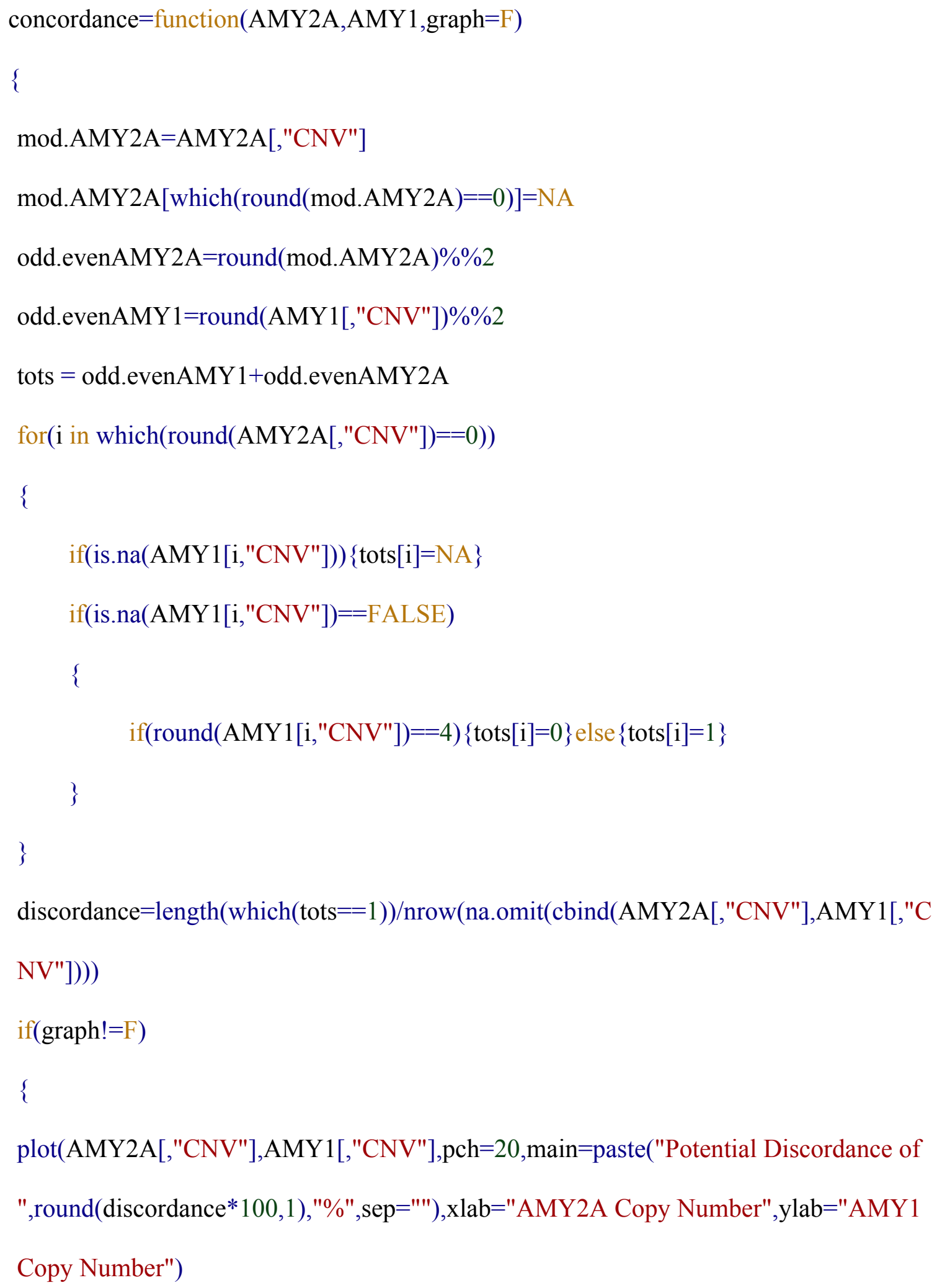




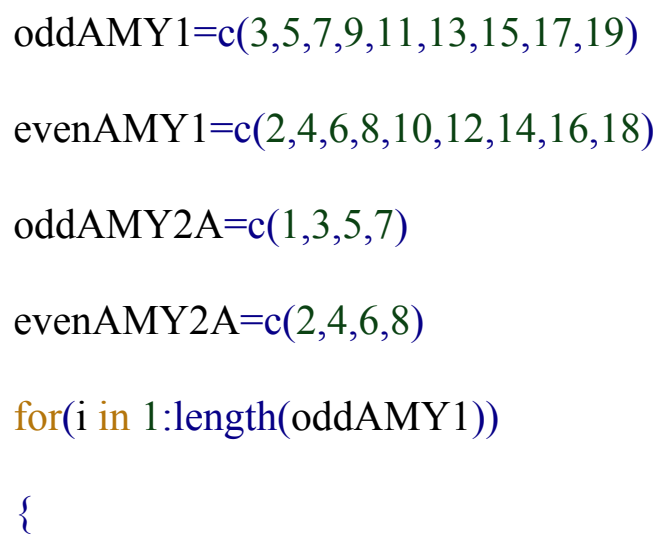


$\operatorname{rect}($ evenAMY2A[4]-0.5,evenAMY1[i]-

0.5 ,evenAMY2A[4]+0.5,evenAMY1[i]+0.5,col="light gray")

\}

segments(x0=AMY2A[,"PoissonCNVMin"],x1=AMY2A[,"PoissonCNVMax"],y0=AM Y1[,"CNV"],col="pink")

segments(y0=AMY1[,"PoissonCNVMin"],y1=AMY1[,"PoissonCNVMax"],x0=AMY2

$\mathrm{A}[, " \mathrm{CNV} "], \mathrm{col}="$ pink")

points(AMY2A[,"CNV"],AMY1[,"CNV"],pch=20)

\}

tots $=$ as.character(tots)

$\operatorname{tots}[$ which $($ tots $==0 \mid$ tots $==2)]=$ TRUE

$\operatorname{tots}[$ which $($ tots $==1)]=$ FALSE

return(tots)

\}

\#This takes the concordance to the next level by selecting which of your two copy

number calls most fit with your AMY2A call. If

\#both fit, then it averages. If neither are concordant, then it averages.

Concordance.Averager $=$

function(AMY1.matrix,AMY1.redo.matrix,concordant.AMY1,concordant.AMY1.redo)

\{

concordance.average=cbind(AMY1.matrix[,"CNV"],AMY1.redo.matrix[,"CNV"],AMY1 .matrix[,"CNV"],AMY1.redo.matrix[,"CNV"])

colnames $($ concordance.average $)=c($ adjusted CNV concentrated","adjusted CNV 
dilute","CNV concentrated","CNV dilute")

rownames $($ concordance.average)=AMY1.matrix[,"Well"]

\#Getting rid of discordant samples

concordance.average[which(concordant.AMY1=="FALSE"),"adjusted CNV

concentrated"]=NA

concordance.average[which(concordant.AMY1.redo=="FALSE"),"adjusted CNV

dilute"]=NA

\#Average

average=rowMeans(concordance.average[,c("adjusted CNV concentrated","adjusted

CNV dilute")],na.rm=T)

average[is.na(average)]=rowMeans(concordance.average[is.na(average),c("CNV

concentrated","CNV dilute")],na.rm=T)

\#confidence intervals

conf.dil=concordance.average[,"adjusted CNV dilute"]-

AMY1.redo.matrix[,"PoissonCNVMin"]

conf.conc=concordance.average[,"adjusted CNV concentrated"]-

AMY1.matrix[,"PoissonCNVMin"]

$\operatorname{conf}=\left(\left(\operatorname{rowSums}\left(\operatorname{cbind}\left(\operatorname{conf}_{\text {dil }}{ }^{\wedge} 2, \text { conf. } \operatorname{conc}^{\wedge} 2\right), \text { na.rm }=T\right)^{\wedge} 0.5\right)\right) /(4-$

(as.numeric(as.factor(is.na(concordance.average[,"adjusted CNV

concentrated"])))+as.numeric(as.factor(is.na(concordance.average[,"adjusted CNV

dilute"])))))

concordance. average. total $=$ cbind(average, average-conf,average + conf)

colnames $($ concordance.average.total)=c("CNV","PoissonCNVMin","PoissonCNVMax") 
return(concordance.average.total)

\}

\#This is where you do your work

\#Load in your csv from QuantaSoft using this command. The big graph contains your corrected values. The three smaller graphs display the work the programs are doing in order to find a correction factor. This is the plate-wide correction factor that will bring the majority of your calls closer to integers. It is calculated only from those samples that have tight confidence intervals, but is applied to the entire plate

AMY2B.2=load.in(file="/Users/cusher/Desktop/Dropbox/QLPs/GoT2D_AMY2B_201412-30-00-59/GoT2D_AMY2B.csv",graph=T)

AMY2B.1=load.in(file="/Users/cusher/Desktop/Dropbox/QLPs/GoT2D_Plate1_for_real _2014-12-30-01-02/GoT2D_Plate1_for_real.csv",graph=T)

\#The first histogram contains your DNA concentration values for the ddPCR. The second histogram is a hypothetical one calculated from the 'afford' value. The afford value is how much more DNA you could put in the reaction if you had to (if you put $3 \mathrm{ul}$ in this reaction, you could hypothetically afford to put in $3 \mathrm{x}$ more into the next one before you hit the limit of $9 u$ in a reaction. The bin value simply adjusts the histogram. The amy value is saying whether this reaction was for AMY1 or AMY2A/B. The third graph in this series is displaying the well positions of samples that failed to have enough DNA in this reaction. This graph helps to see if an entire row failed, and the graph should remain mostly constant in the other reactions, unless the plate is accidentally flipped.) concentrations(X=AMY2B.1[,"conc.ng.ul"],afford=2.47,bins=100,amy=2,graph=T) concentrations(X=AMY2B.2[,"conc.ng.ul"], afford=2.47,bins=100,amy=2, graph=T) 
concentration.slider(X=AMY2B.1[,"conc.ng.ul"], $\min =0$, $\max =2.47$, input=3.6, amy=2, gra $\mathrm{ph}=\mathrm{T})$

concentration.slider(X=AMY2B.1[,"conc.ng.ul"], $\min =0, \max =2.47$, input=3.6, amy=1, gra $\mathrm{ph}=\mathrm{T})$

concentration.slider(X=AMY2B.2[,"conc.ng.ul"], $\min =0$, $\max =2.47$, input=3.6, amy=1, gra $\mathrm{ph}=\mathrm{T})$

concentration.slider(X=AMY2B.2[,"conc.ng.ul"], $\min =0$, $\max =2.47$, input=3.6, amy=2, gra $\mathrm{ph}=\mathrm{T})$

\#More of the same for AMY2A reaction

AMY2A=load.in(file=" $\sim$ Desktop/Quantalife Runs

Amylase/BMI/Plate3_Estonian_BMI_AMY2A/Plate3_Estonian_BMI_AMY2A.csv",gr $\mathrm{aph}=\mathrm{T})$

concentrations $(\mathrm{X}=\mathrm{AMY} 2 \mathrm{~A}[$,"conc.ng.ul"],afford=2.47,bins=100,amy=2,graph=T)

AMY2A=load.in(file=" $/$ Desktop/Dropbox/qlps/AMY2A_InChianti_2014-10-28-2126/AMY2A_InChianti.csv",graph=T)

concentrations $(\mathrm{X}=\mathrm{AMY} 2 \mathrm{~A}[$,"conc.ng.ul"],afford=2.47, bins=100, amy=2, graph=T,plate=1 1)

\#This graph helps you to decide how much sample to put into the AMY1 reaction based on the AMY2A reaction. You want to pick an input at where the green line levels off. The input value is how much sample (ul) that you put into the AMY2A reaction. The min and max are the multiples that you could put into the AMY1 reaction (you don't really have to change those). The amy value is 1 if you're using this function to predict for an AMY1 reaction. 
concentration.slider $(\mathrm{X}=\mathrm{AMY} 2 \mathrm{~A}[$,"conc.ng.ul"], $\min =0, \max =2.47$,input=3.6, amy=1, graph $=\mathrm{T})$

\#More of the same for the AMY1 reaction

AMY1=load.in(file="/Users/cusher/Desktop/Dropbox/QLPs/AMY1_plate1_2014-12-30-

18-36/AMY1_plate1.csv", graph=T)

concentrations $(\mathrm{X}=\mathrm{AMY} 1[$,"conc.ng.ul"],afford=1,bins=50,amy=1,graph=T)

AMY1.2=load.in(file="/Users/cusher/Desktop/Dropbox/QLPs/AMY1_plate2_2014-12-

30-19-45/AMY1_plate2.csv", graph=T)

concentrations $(\mathrm{X}=\mathrm{AMY} 1.2[$,"conc.ng.ul"],afford=1,bins=50, amy=1,graph=T,plate=11)

\#Gives you a graph of the concordance between the AMY1 values and the AMY2A

values. Gray boxes are expected clusterings. Percentage given at top is a rough

approximation of how accurate the assays are being.

concordant=concordance $(\mathrm{AMY} 1=\mathrm{AMY} 1, \mathrm{AMY} 2 \mathrm{~A}=\mathrm{AMY} 2 \mathrm{~A}, \mathrm{graph}=\mathrm{T})$

\#Now that you've done one AMY1 reaction, it's time to do another one. This time, you're trying to salvage the high copy number samples that botched their last assay because they oversaturated the reaction with FAM. The idea behind this graph is much the same as the other one: pick the concentration with the highest peak.

Percent.slider $(\mathrm{x}=\mathrm{AMY} 1.2, \mathrm{~min}=0, \mathrm{max}=2.6$,input $=4.3$, graph $=\mathrm{T})$

\#Doing much the same with the second AMY1 reaction

AMY1.redo=load.in(file=" $/$ Desktop/Quantalife Runs

Amylase/BMI/Plate3_Estonian_BMI_AMY1_redo/Plate3_Estonian_BMI_AMY1_redo. $\left.\operatorname{csv}^{\prime \prime}, \mathrm{g}=\mathrm{T}\right)$

AMY1.redo=load.in(file=" $/$ Desktop/Dropbox/QLPs/Extend_AMY1_2nd_run_2014-10- 
31-11-09/Extend_AMY1_2nd_run.csv",g=T)

concentrations $(\mathrm{X}=\mathrm{AMY} 1 \cdot$ redo[,"conc.ng.ul"],afford=1, bins=50, amy=1, graph=T)

concordant.redo $=$ concordance $(\mathrm{AMY} 1=\mathrm{AMY} 1 \cdot$ redo, $\mathrm{AMY} 2 \mathrm{~A}=\mathrm{AMY} 2 \mathrm{~A}, \mathrm{~g}=\mathrm{T})$

\#Spits out the AMY2A-informed averages of AMY1 copy number

AMY1.Informed.With.AMY2A.Copy.Number=Concordance.Averager(AMY1,AMY1.re do,concordant,concordant.redo) 


\section{References}

1. Conrad, D.F. et al. Origins and functional impact of copy number variation in the human genome. Nature 464, 704-12 (2010).

2. Inoue, K. \& Lupski, J.R. Molecular mechanisms for genomic disorders. Annu Rev Genomics Hum Genet 3, 199-242 (2002).

3. Lupski, J.R. Genomic disorders ten years on. Genome Med 1, 42 (2009).

4. Padiath, Q.S. et al. Lamin B1 duplications cause autosomal dominant leukodystrophy. Nat Genet 38, 1114-23 (2006).

5. Le Marechal, C. et al. Hereditary pancreatitis caused by triplication of the trypsinogen locus. Nat Genet 38, 1372-4 (2006).

6. Lee, J.A. \& Lupski, J.R. Genomic rearrangements and gene copy-number alterations as a cause of nervous system disorders. Neuron 52, 103-21 (2006).

7. McCarroll, S.A. \& Altshuler, D.M. Copy-number variation and association studies of human disease. Nat Genet 39, S37-42 (2007).

8. Stankiewicz, P. \& Lupski, J.R. Structural variation in the human genome and its role in disease. Annu Rev Med 61, 437-55 (2010).

9. Malhotra, D. \& Sebat, J. CNVs: harbingers of a rare variant revolution in psychiatric genetics. Cell 148, 1223-41 (2012).

10. Stankiewicz, P.\& Lupski, J.R. The genomic basis of disease, mechanisms and assays for genomic disorders. Genome Dyn 1, 1-16 (2006).

11. McCarroll, S.A. et al. Integrated detection and population-genetic analysis of SNPs and copy number variation. Nat Genet 40, 1166-74 (2008). 
12. Redon, R. et al. Global variation in copy number in the human genome. Nature 444, 444-54 (2006).

13. Wellcome Trust Case Control, C. Genome-wide association study of 14,000 cases of seven common diseases and 3,000 shared controls. Nature 447, 661-78 (2007).

14. Browning, S.R. Missing data imputation and haplotype phase inference for genome-wide association studies. Hum Genet 124, 439-50 (2008).

15. Weischenfeldt, J., Symmons, O., Spitz, F. \& Korbel, J.O. Phenotypic impact of genomic structural variation: insights from and for human disease. Nat Rev Genet 14, 125-38 (2013).

16. Handsaker, R.E. et al. Large multiallelic copy number variations in humans. Nat Genet (2015).

17. Perry, G.H. et al. Copy number variation and evolution in humans and chimpanzees. Genome Res 18, 1698-710 (2008).

18. Perry, G.H. et al. Diet and the evolution of human amylase gene copy number variation. Nat Genet 39, 1256-60 (2007).

19. Sudmant, P.H. et al. Diversity of human copy number variation and multicopy genes. Science 330, 641-6 (2010).

20. Perry, G.H. et al. The fine-scale and complex architecture of human copy-number variation. Am J Hum Genet 82, 685-95 (2008).

21. Boettger, L.M., Handsaker, R.E., Zody, M.C. \& McCarroll, S.A. Structural haplotypes and recent evolution of the human 17q21.31 region. Nat Genet 44, 881-5 (2012).

22. Steinberg, K.M. et al. Structural diversity and African origin of the $17 \mathrm{q} 21.31$ inversion polymorphism. Nat Genet 44, 872-80 (2012).

23. Stefansson, H. et al. A common inversion under selection in Europeans. Nat Genet 37, 129-37 (2005). 
24. Skipper, L. et al. Linkage disequilibrium and association of MAPT H1 in Parkinson disease. Am J Hum Genet 75, 669-77 (2004).

25. Simon-Sanchez, J. et al. Genome-wide association study reveals genetic risk underlying Parkinson's disease. Nat Genet 41, 1308-12 (2009).

26. Niederer, H.A. et al. Copy number, linkage disequilibrium and disease association in the FCGR locus. Hum Mol Genet 19, 3282-94 (2010).

27. Mason, M.J. et al. Low HERV-K(C4) copy number is associated with type 1 diabetes. Diabetes 63, 1789-95 (2014).

28. Falchi, M. et al. Low copy number of the salivary amylase gene predisposes to obesity. Nat Genet 46, 492-7 (2014).

29. Aklillu, E. et al. CCL3L1 copy number, HIV load, and immune reconstitution in sub-Saharan Africans. BMC Infect Dis 13, 536 (2013).

30. Yamanaka, M., Kato, Y., Angata, T. \& Narimatsu, H. Deletion polymorphism of SIGLEC14 and its functional implications. Glycobiology 19, 841-6 (2009).

31. Schlattl, A., Anders, S., Waszak, S.M., Huber, W. \& Korbel, J.O. Relating CNVs to transcriptome data at fine resolution: assessment of the effect of variant size, type, and overlap with functional regions. Genome Res 21, 2004-13 (2011).

32. Innan, H. \& Kondrashov, F. The evolution of gene duplications: classifying and distinguishing between models. Nat Rev Genet 11, 97-108 (2010).

33. Stranger, B.E. et al. Relative impact of nucleotide and copy number variation on gene expression phenotypes. Science 315, 848-53 (2007).

34. Robert E. Handsaker, V.V.D., Jennifer R. Berman, Giulio Genovese, Seva Kashin, Linda M. Boettger, Steven A. McCarroll Large multi-allelic copy number variations in humans. (2015).

35. Pruitt, K.D. et al. RefSeq: an update on mammalian reference sequences. Nucleic Acids Res 42, D756-63 (2014). 
36. Chen, J.Y. et al. Association of FCGR3A and FCGR3B Copy Number Variations With Systemic Lupus Erythematosus and Rheumatoid Arthritis in Taiwanese Patients. Arthritis Rheumatol 66, 3113-21 (2014).

37. Fanciulli, M. et al. FCGR3B copy number variation is associated with susceptibility to systemic, but not organ-specific, autoimmunity. Nat Genet 39, 721-3 (2007).

38. Mamtani, M., Anaya, J.M., He, W. \& Ahuja, S.K. Association of copy number variation in the FCGR3B gene with risk of autoimmune diseases. Genes Immun 11, 155-60 (2010).

39. McKinney, C. et al. Association of variation in Fcgamma receptor 3B gene copy number with rheumatoid arthritis in Caucasian samples. Ann Rheum Dis 69, 17116 (2010).

40. Allen, S.J. et al. alpha+-Thalassemia protects children against disease caused by other infections as well as malaria. Proc Natl Acad Sci U S A 94, 14736-41 (1997).

41. Arason, G.J. et al. Smoking and a complement gene polymorphism interact in promoting cardiovascular disease morbidity and mortality. Clin Exp Immunol 149, 132-8 (2007).

42. Gonzalez, E. et al. The influence of CCL3L1 gene-containing segmental duplications on HIV-1/AIDS susceptibility. Science 307, 1434-40 (2005).

43. Yang, Y. et al. Gene copy-number variation and associated polymorphisms of complement component $\mathrm{C} 4$ in human systemic lupus erythematosus (SLE): low copy number is a risk factor for and high copy number is a protective factor against SLE susceptibility in European Americans. Am J Hum Genet 80, 1037-54 (2007).

44. Hollox, E.J. et al. Psoriasis is associated with increased beta-defensin genomic copy number. Nat Genet 40, 23-5 (2008).

45. Burns, J.C. et al. Genetic variations in the receptor-ligand pair CCR5 and CCL3L1 are important determinants of susceptibility to Kawasaki disease. $J$ Infect Dis 192, 344-9 (2005). 
46. McKinney, C. et al. Evidence for an influence of chemokine ligand 3-like 1 (CCL3L1) gene copy number on susceptibility to rheumatoid arthritis. Ann Rheum Dis 67, 409-13 (2008).

47. Fellermann, K. et al. A chromosome 8 gene-cluster polymorphism with low human beta-defensin 2 gene copy number predisposes to Crohn disease of the colon. Am J Hum Genet 79, 439-48 (2006).

48. Cantsilieris, S. \& White, S.J. Correlating multiallelic copy number polymorphisms with disease susceptibility. Hum Mutat 34, 1-13 (2013).

49. Carpenter, D., Walker, S., Prescott, N., Schalkwijk, J. \& Armour, J.A. Accuracy and differential bias in copy number measurement of CCL3L1 in association studies with three auto-immune disorders. BMC Genomics 12, 418 (2011).

50. Aldhous, M.C. et al. Measurement methods and accuracy in copy number variation: failure to replicate associations of beta-defensin copy number with Crohn's disease. Hum Mol Genet 19, 4930-8 (2010).

51. Aitman, T.J. et al. Copy number polymorphism in Fcgr3 predisposes to glomerulonephritis in rats and humans. Nature 439, 851-5 (2006).

52. Haldorsen, K. et al. No association of primary Sjogren's syndrome with Fcgamma receptor gene variants. Genes Immun 14, 234-7 (2013).

53. Molokhia, M. et al. FCGR3B copy number variation is associated with systemic lupus erythematosus risk in Afro-Caribbeans. Rheumatology (Oxford) 50, 120610 (2011).

54. Morris, D.L. et al. Evidence for both copy number and allelic (NA1/NA2) risk at the FCGR3B locus in systemic lupus erythematosus. Eur J Hum Genet 18, 1027$31(2010)$.

55. Willcocks, L.C. et al. Copy number of FCGR3B, which is associated with systemic lupus erythematosus, correlates with protein expression and immune complex uptake. J Exp Med 205, 1573-82 (2008). 
56. Lester, S., Nossent, J. \& Rischmueller, M. Comment on 'no association of primary Sjogren's syndrome with Fcgamma receptor gene variants'. Genes Immun 14, 5301 (2013).

57. Wellcome Trust Case Control, C. et al. Genome-wide association study of CNVs in 16,000 cases of eight common diseases and 3,000 shared controls. Nature 464, 713-20 (2010).

58. Breunis, W.B. et al. Copy number variation at the FCGR locus includes FCGR3A, FCGR2C and FCGR3B but not FCGR2A and FCGR2B. Hum Mutat 30, E640-50 (2009).

59. Marques, R.B. et al. Genetic variation of the Fc gamma receptor 3B gene and association with rheumatoid arthritis. PLoS One 5(2010).

60. Robinson, J.I. et al. Confirmation of association of FCGR3B but not FCGR3A copy number with susceptibility to autoantibody positive rheumatoid arthritis. Hum Mutat 33, 741-9 (2012).

61. Thabet, M.M. et al. Contribution of Fcgamma receptor IIIA gene $158 \mathrm{~V} / \mathrm{F}$ polymorphism and copy number variation to the risk of ACPA-positive rheumatoid arthritis. Ann Rheum Dis 68, 1775-80 (2009).

62. Stuart, P.E. et al. Association of beta-defensin copy number and psoriasis in three cohorts of European origin. J Invest Dermatol 132, 2407-13 (2012).

63. Bentley, R.W. et al. Association of higher DEFB4 genomic copy number with Crohn's disease. Am J Gastroenterol 105, 354-9 (2010).

64. Blasko, B. et al. Low complement C4B gene copy number predicts short-term mortality after acute myocardial infarction. Int Immunol 20, 31-7 (2008).

65. Boteva, L. et al. Genetically determined partial complement C4 deficiency states are not independent risk factors for SLE in UK and Spanish populations. Am J Hum Genet 90, 445-56 (2012).

66. Lv, J. et al. FCGR3B copy number variation is not associated with lupus nephritis in a Chinese population. Lupus 19, 158-61 (2010). 
67. Lv, Y. et al. Confirmation of $\mathrm{C} 4$ gene copy number variation and the association with systemic lupus erythematosus in Chinese Han population. Rheumatol Int 32, 3047-53 (2012).

68. Pani, M.A. et al. The variable endogenous retroviral insertion in the human complement $\mathrm{C} 4$ gene: a transmission study in type I diabetes mellitus. Hum Immunol 63, 481-4 (2002).

69. Mockenhaupt, F.P. et al. Alpha(+)-thalassemia protects African children from severe malaria. Blood 104, 2003-6 (2004).

70. Lell, B. et al. The role of red blood cell polymorphisms in resistance and susceptibility to malaria. Clin Infect Dis 28, 794-9 (1999).

71. May, J. et al. Hemoglobin variants and disease manifestations in severe falciparum malaria. JAMA 297, 2220-6 (2007).

72. Williams, T.N. et al. Both heterozygous and homozygous alpha+ thalassemias protect against severe and fatal Plasmodium falciparum malaria on the coast of Kenya. Blood 106, 368-71 (2005).

73. Bhattacharya, T. et al. CCL3L1 and HIV/AIDS susceptibility. Nat Med 15, 11125 (2009).

74. Dolan, M.J. et al. CCL3L1 and CCR5 influence cell-mediated immunity and affect HIV-AIDS pathogenesis via viral entry-independent mechanisms. Nat Immunol 8, 1324-36 (2007).

75. Huik, K. et al. CCL3L1 copy number is a strong genetic determinant of HIV seropositivity in Caucasian intravenous drug users. J Infect Dis 201, 730-9 (2010).

76. Kuhn, L. et al. African infants' CCL3 gene copies influence perinatal HIV transmission in the absence of maternal nevirapine. AIDS 21, 1753-61 (2007).

77. Larsen, M.H. et al. CCL3L gene copy number and survival in an HIV-1 infected Zimbabwean population. Infect Genet Evol 12, 1087-93 (2012). 
78. Lee, E.Y. et al. The impact of CCL3L1 copy number in an HIV-1-infected white population. AIDS 24, 1589-91 (2010).

79. Meddows-Taylor, S. et al. Reduced ability of newborns to produce CCL3 is associated with increased susceptibility to perinatal human immunodeficiency virus 1 transmission. J Gen Virol 87, 2055-65 (2006).

80. Nakajima, T. et al. Copy number variations of CCL3L1 and long-term prognosis of HIV-1 infection in asymptomatic HIV-infected Japanese with hemophilia. Immunogenetics 59, 793-8 (2007).

81. Rathore, A. et al. Association of CCR5-59029 A/G and CCL3L1 copy number polymorphism with HIV type 1 transmission/progression among HIV type 1seropositive and repeatedly sexually exposed HIV type 1-seronegative North Indians. AIDS Res Hum Retroviruses 25, 1149-56 (2009).

82. Shao, W. et al. CCL3L1 and CCL4L1: variable gene copy number in adolescents with and without human immunodeficiency virus type 1 (HIV-1) infection. Genes Immun 8, 224-31 (2007).

83. Shostakovich-Koretskaya, L. et al. Combinatorial content of CCL3L and CCL4L gene copy numbers influence HIV-AIDS susceptibility in Ukrainian children. AIDS 23, 679-88 (2009).

84. Urban, T.J. et al. CCL3L1 and HIV/AIDS susceptibility. Nat Med 15, 1110-2 (2009).

85. Kim, H.E. et al. Variations in the number of CCL3L1 gene copies and Kawasaki disease in Korean children. Pediatr Cardiol 33, 1259-63 (2012).

86. Nordang, G.B. et al. Association analysis of the CCL3L1 copy number locus by paralogue ratio test in Norwegian rheumatoid arthritis patients and healthy controls. Genes Immun 13, 579-82 (2012).

87. Mamtani, M. et al. Association of CCR2-CCR5 haplotypes and CCL3L1 copy number with Kawasaki Disease, coronary artery lesions, and IVIG responses in Japanese children. PLoS One 5, e11458 (2010). 
88. Korn, J.M. et al. Integrated genotype calling and association analysis of SNPs, common copy number polymorphisms and rare CNVs. Nat Genet 40, 1253-60 (2008).

89. Field, S.F. et al. Experimental aspects of copy number variant assays at CCL3L1. Nat Med 15, 1115-7 (2009).

90. Fode, P. et al. Determination of beta-defensin genomic copy number in different populations: a comparison of three methods. PLoS One 6, e16768 (2011).

91. Collins, A. Allelic association: linkage disequilibrium structure and gene mapping. Mol Biotechnol 41, 83-9 (2009).

92. Slatkin, M. Linkage disequilibrium--understanding the evolutionary past and mapping the medical future. Nat Rev Genet 9, 477-85 (2008).

93. Ragoussis, J. Genotyping technologies for genetic research. Annu Rev Genomics Hum Genet 10, 117-33 (2009).

94. Speliotes, E.K. et al. Association analyses of 249,796 individuals reveal 18 new loci associated with body mass index. Nat Genet 42, 937-48 (2010).

95. Groot, P.C. et al. The human alpha-amylase multigene family consists of haplotypes with variable numbers of genes. Genomics 5, 29-42 (1989).

96. Groot, P.C., Mager, W.H. \& Frants, R.R. Interpretation of polymorphic DNA patterns in the human alpha-amylase multigene family. Genomics 10, 779-85 (1991).

97. Ramasubbu, N., Paloth, V., Luo, Y., Brayer, G.D. \& Levine, M.J. Structure of human salivary alpha-amylase at 1.6 A resolution: implications for its role in the oral cavity. Acta Crystallogr D Biol Crystallogr 52, 435-46 (1996).

98. Bank, R.A., Hettema, E.H., Arwert, F., Amerongen, A.V. \& Pronk, J.C. Electrophoretic characterization of posttranslational modifications of human parotid salivary alpha-amylase. Electrophoresis 12, 74-9 (1991). 
99. Takashima, S.a.A., J. . Glycosylation and secretion of human $\alpha$-amylases. Advances in Biological Chemistry (2012).

100. Ramasubbu, N., Ragunath, C. \& Mishra, P.J. Probing the role of a mobile loop in substrate binding and enzyme activity of human salivary amylase. J Mol Biol 325, 1061-76 (2003).

101. Bush, D.S., Sticher, L., van Huystee, R., Wagner, D. \& Jones, R.L. The calcium requirement for stability and enzymatic activity of two isoforms of barley aleurone alpha-amylase. J Biol Chem 264, 19392-8 (1989).

102. Numao, S. et al. Probing the role of the chloride ion in the mechanism of human pancreatic alpha-amylase. Biochemistry 41, 215-25 (2002).

103. Maurus, R. et al. Structural and mechanistic studies of chloride induced activation of human pancreatic alpha-amylase. Protein Sci 14, 743-55 (2005).

104. Brayer, G.D., Luo, Y. \& Withers, S.G. The structure of human pancreatic alphaamylase at 1.8 A resolution and comparisons with related enzymes. Protein Sci 4, 1730-42 (1995).

105. Brayer, G.D. et al. Subsite mapping of the human pancreatic alpha-amylase active site through structural, kinetic, and mutagenesis techniques. Biochemistry 39, 4778-91 (2000).

106. Ramasubbu, N. et al. Human salivary alpha-amylase Trp58 situated at subsite -2 is critical for enzyme activity. Eur J Biochem 271, 2517-29 (2004).

107. Kanehisa, M. \& Goto, S. KEGG: kyoto encyclopedia of genes and genomes. Nucleic Acids Res 28, 27-30 (2000).

108. Samuelson, L.C., Wiebauer, K., Gumucio, D.L. \& Meisler, M.H. Expression of the human amylase genes: recent origin of a salivary amylase promoter from an actin pseudogene. Nucleic Acids Res 16, 8261-76 (1988).

109. Seyama, K., Nukiwa, T., Takahashi, K., Takahashi, H. \& Kira, S. Amylase mRNA transcripts in normal tissues and neoplasms: the implication of different expressions of amylase isogenes. J Cancer Res Clin Oncol 120, 213-20 (1994). 
110. Consortium, G.T. The Genotype-Tissue Expression (GTEx) project. Nat Genet 45, 580-5 (2013).

111. Lukk, M. et al. A global map of human gene expression. Nat Biotechnol 28, 3224 (2010).

112. Watanabe, T. et al. Comparison of lung cancer cell lines representing four histopathological subtypes with gene expression profiling using quantitative realtime PCR. Cancer Cell Int 10, 2 (2010).

113. Chaudhuri, B., Rojek, J., Vickerman, M.M., Tanzer, J.M. \& Scannapieco, F.A. Interaction of salivary alpha-amylase and amylase-binding-protein A (AbpA) of Streptococcus gordonii with glucosyltransferase of S. gordonii and Streptococcus mutans. BMC Microbiol 7, 60 (2007).

114. Rosenblum, J.L., Irwin, C.L. \& Alpers, D.H. Starch and glucose oligosaccharides protect salivary-type amylase activity at acid pH. Am J Physiol 254, G775-80 (1988).

115. Sevenhuysen, G.P., Holodinsky, C. \& Dawes, C. Development of salivary alphaamylase in infants from birth to 5 months. Am J Clin Nutr 39, 584-8 (1984).

116. Brown, N.J. et al. Biological markers of stress in pediatric acute burn injury. Burns 40, 887-95 (2014).

117. Out, D., Bakermans-Kranenburg, M.J., Granger, D.A., Cobbaert, C.M. \& van Ijzendoorn, M.H. State and trait variance in salivary alpha-amylase: a behavioral genetic study. Biol Psychol 88, 147-54 (2011).

118. Out, D., Granger, D.A., Sephton, S.E. \& Segerstrom, S.C. Disentangling sources of individual differences in diurnal salivary alpha-amylase: reliability, stability and sensitivity to context. Psychoneuroendocrinology 38, 367-75 (2013).

119. Keller, P.S., El-Sheikh, M., Granger, D.A. \& Buckhalt, J.A. Interactions between salivary cortisol and alpha-amylase as predictors of children's cognitive functioning and academic performance. Physiol Behav 105, 987-95 (2012).

120. Nater, U.M. et al. Human salivary alpha-amylase reactivity in a psychosocial stress paradigm. Int J Psychophysiol 55, 333-42 (2005). 
121. Tanaka, Y. et al. Salivary alpha-amylase and cortisol responsiveness following electrically stimulated physical stress in bipolar disorder patients. Neuropsychiatr Dis Treat 8, 1899-905 (2013).

122. Tanaka, Y. et al. Salivary alpha-amylase and cortisol responsiveness following electrical stimulation stress in panic disorder patients. Neurosci Res 73, 80-4 (2012).

123. Adam, E.K., Till Hoyt, L. \& Granger, D.A. Diurnal alpha amylase patterns in adolescents: associations with puberty and momentary mood states. Biol Psychol 88, 170-3 (2011).

124. Segal, S.K., Cotman, C.W. \& Cahill, L.F. Exercise-induced noradrenergic activation enhances memory consolidation in both normal aging and patients with amnestic mild cognitive impairment. J Alzheimers Dis 32, 1011-8 (2012).

125. Jain, S. et al. Amylase alpha-1A (AMY1A): a novel immunohistochemical marker to differentiate chromophobe renal cell carcinoma from benign oncocytoma. Am J Surg Pathol 37, 1824-30 (2013).

126. Kang, Y. Psychological stress-induced changes in salivary alpha-amylase and adrenergic activity. Nurs Health Sci 12, 477-84 (2010).

127. Endo, T. et al. Amylase alpha-2A autoantibodies: novel marker of autoimmune pancreatitis and fulminant type 1 diabetes. Diabetes 58, $732-7$ (2009).

128. Scannapieco, F.A., Bergey, E.J., Reddy, M.S. \& Levine, M.J. Characterization of salivary alpha-amylase binding to Streptococcus sanguis. Infect Immun 57, 285363 (1989).

129. Douglas, C.W. Characterization of the alpha-amylase receptor of Streptococcus gordonii NCTC 7868. J Dent Res 69, 1746-52 (1990).

130. Kilian, M. \& Nyvad, B. Ability to bind salivary alpha-amylase discriminates certain viridans group streptococcal species. J Clin Microbiol 28, 2576-7 (1990).

131. Gwynn, J.P. \& Douglas, C.W. Comparison of amylase-binding proteins in oral streptococci. FEMS Microbiol Lett 124, 373-9 (1994). 
132. Spear, G.T. et al. Human alpha-amylase present in lower-genital-tract mucosal fluid processes glycogen to support vaginal colonization by Lactobacillus. $J$ Infect Dis 210, 1019-28 (2014).

133. Read, N.W. et al. Swallowing food without chewing; a simple way to reduce postprandial glycaemia. Br J Nutr 55, 43-7 (1986).

134. Mandel, A.L. \& Breslin, P.A. High endogenous salivary amylase activity is associated with improved glycemic homeostasis following starch ingestion in adults. J Nutr 142, 853-8 (2012).

135. Bailey, J.N. et al. The Role of Copy Number Variation in African Americans with Type 2 Diabetes-Associated End Stage Renal Disease. J Mol Genet Med 7, 61 (2013).

136. Mandel, A.L., Peyrot des Gachons, C., Plank, K.L., Alarcon, S. \& Breslin, P.A. Individual differences in AMY1 gene copy number, salivary alpha-amylase levels, and the perception of oral starch. PLoS One 5, e13352 (2010).

137. Santos, J.L. et al. Copy number polymorphism of the salivary amylase gene: implications in human nutrition research. J Nutrigenet Nutrigenomics 5, 117-31 (2012).

138. Layer, P., Rizza, R.A., Zinsmeister, A.R., Carlson, G.L. \& DiMagno, E.P. Effect of a purified amylase inhibitor on carbohydrate tolerance in normal subjects and patients with diabetes mellitus. Mayo Clin Proc 61, $442-7$ (1986).

139. Udani, J.K., Singh, B.B., Barrett, M.L. \& Preuss, H.G. Lowering the glycemic index of white bread using a white bean extract. Nutr J 8, 52 (2009).

140. Barrett, M.L. \& Udani, J.K. A proprietary alpha-amylase inhibitor from white bean (Phaseolus vulgaris): a review of clinical studies on weight loss and glycemic control. Nutr J 10, 24 (2011).

141. Ogawa, H. \& Date, K. The "white kidney bean incident" in Japan. Methods Mol Biol 1200, 39-45 (2014).

142. Sjolund, K. et al. Selective deficiency of pancreatic amylase. Gut 32, 546-8 (1991). 
143. Singh, S. \& Guruprasad, L. Structure and sequence based analysis of alphaamylase evolution. Protein Pept Lett 21, 948-56 (2014).

144. Samuelson, L.C., Phillips, R.S. \& Swanberg, L.J. Amylase gene structures in primates: retroposon insertions and promoter evolution. Mol Biol Evol 13, 767-79 (1996).

145. Groot, P.C. et al. Evolution of the human alpha-amylase multigene family through unequal, homologous, and inter- and intrachromosomal crossovers. Genomics 8, 97-105 (1990).

146. Zhang, F., Gu, W., Hurles, M.E. \& Lupski, J.R. Copy number variation in human health, disease, and evolution. Annu Rev Genomics Hum Genet 10, 451-81 (2009).

147. Axelsson, E. et al. The genomic signature of dog domestication reveals adaptation to a starch-rich diet. Nature 495, 360-4 (2013).

148. Hagenbuchle, O., Bovey, R. \& Young, R.A. Tissue-specific expression of mousealpha-amylase genes: nucleotide sequence of isoenzyme mRNAs from pancreas and salivary gland. Cell 21, 179-87 (1980).

149. Kent, W.J. et al. The human genome browser at UCSC. Genome Res 12, 9961006 (2002).

150. Bult, C.J. et al. The Mouse Genome Database (MGD): mouse biology and model systems. Nucleic Acids Res 36, D724-8 (2008).

151. Meisler, M.H., Antonucci, T.K., Treisman, L.O., Gumucio, D.L. \& Samuelson, L.C. Interstrain variation in amylase gene copy number and mRNA abundance in three mouse tissues. Genetics 113, 713-22 (1986).

152. Thomsen, K.K., Hjorth, J.P. \& Nielsen, J.T. Genetic variation in restriction patterns among mouse amylase gene complexes. Biochem Genet 22, 257-73 (1984).

153. Behringer, V. et al. Measurements of salivary alpha amylase and salivary cortisol in hominoid primates reveal within-species consistency and between-species differences. PLoS One 8, e60773 (2013). 
154. Hindson, B.J. et al. High-throughput droplet digital PCR system for absolute quantitation of DNA copy number. Anal Chem 83, 8604-10 (2011).

155. Mazaika, E. \& Homsy, J. Digital Droplet PCR: CNV Analysis and Other Applications. Curr Protoc Hum Genet 82, 724 1-7 2413 (2014).

156. Handsaker, R.E., Korn, J.M., Nemesh, J. \& McCarroll, S.A. Discovery and genotyping of genome structural polymorphism by sequencing on a population scale. Nat Genet 43, 269-76 (2011).

157. The 1000 Genomes Project Consortium. An integrated map of genetic variation from 1,092 human genomes. Nature 491, 56-65 (2012).

158. International HapMap Consortium. The International HapMap Project. Nature 426, 789-96 (2003).

159. Larkin, M.A. et al. Clustal W and Clustal X version 2.0. Bioinformatics 23, 29478 (2007).

160. Untergasser, A. et al. Primer3--new capabilities and interfaces. Nucleic Acids Res 40, e115 (2012).

161. Koren, A. et al. Differential relationship of DNA replication timing to different forms of human mutation and variation. Am J Hum Genet 91, 1033-40 (2012).

162. Sudmant, P.H. et al. Diversity of human copy number variation and multicopy genes. Science 330, 641-6 (2010).

163. Gumucio, D.L., Wiebauer, K., Caldwell, R.M., Samuelson, L.C. \& Meisler, M.H. Concerted evolution of human amylase genes. Mol Cell Biol 8, 1197-205 (1988).

164. Teague, B. et al. High-resolution human genome structure by single-molecule analysis. Proc Natl Acad Sci U S A 107, 10848-53 (2010).

165. Lupski, J.R. \& Stankiewicz, P. Genomic disorders: molecular mechanisms for rearrangements and conveyed phenotypes. PLoS Genet 1, e49 (2005). 
166. Locke, A.E. et al. Genetic studies of body mass index yield new insights for obesity biology. Nature 518, 197-206 (2015).

167. Leitsalu, L. et al. Cohort Profile: Estonian Biobank of the Estonian Genome Center, University of Tartu. Int J Epidemiol (2014).

168. Ferrucci, L. et al. Subsystems contributing to the decline in ability to walk: bridging the gap between epidemiology and geriatric practice in the InCHIANTI study. J Am Geriatr Soc 48, 1618-25 (2000).

169. Mirski, A. Metabolism of adipose tissue in vitro. Biochem J 36, 232-41 (1942).

170. Bingham, S.A. et al. Nutritional methods in the European Prospective Investigation of Cancer in Norfolk. Public Health Nutr 4, 847-58 (2001).

171. Spector, T.D. \& Williams, F.M. The UK Adult Twin Registry (TwinsUK). Twin Res Hum Genet 9, 899-906 (2006).

172. Balkau, B., Eschwege, E., Tichet, J. \& Marre, M. Proposed criteria for the diagnosis of diabetes: evidence from a French epidemiological study (D.E.S.I.R.). Diabetes Metab 23, 428-34 (1997).

173. Meyre, D. et al. Genome-wide association study for early-onset and morbid adult obesity identifies three new risk loci in European populations. Nat Genet 41, 1579 (2009).

174. Wen, W. et al. Meta-analysis identifies common variants associated with body mass index in east Asians. Nat Genet 44, 307-11 (2012).

175. Nookaew, I. et al. Adipose tissue resting energy expenditure and expression of genes involved in mitochondrial function are higher in women than in men. $J$ Clin Endocrinol Metab 98, E370-8 (2013).

176. Tognon, G. et al. Mediterranean diet, overweight and body composition in children from eight European countries: cross-sectional and prospective results from the IDEFICS study. Nutr Metab Cardiovasc Dis 24, 205-13 (2014). 
177. Mottus, R. et al. Personality traits and eating habits in a large sample of Estonians. Health Psychol 31, 806-14 (2012).

178. Berndt, S.I. et al. Genome-wide meta-analysis identifies 11 new loci for anthropometric traits and provides insights into genetic architecture. Nat Genet 45, 501-12 (2013).

179. Zanda, M. et al. A genome-wide assessment of the role of untagged copy number variants in type 1 diabetes. PLoS Genet 10, e1004367 (2014).

180. Hindorff, L.A. et al. Potential etiologic and functional implications of genomewide association loci for human diseases and traits. Proc Natl Acad Sci U S A 106, 9362-7 (2009).

181. Genomes Project, C. et al. A map of human genome variation from populationscale sequencing. Nature 467, 1061-73 (2010).

182. Zeller, T. et al. Genetics and beyond--the transcriptome of human monocytes and disease susceptibility. PLOS ONE 5, e10693 (2010).

183. Kasowski, M. et al. Variation in transcription factor binding among humans. Science 328, 232-5 (2010).

184. Maurano, M.T., Wang, H., Kutyavin, T. \& Stamatoyannopoulos, J.A. Widespread site-dependent buffering of human regulatory polymorphism. PLoS Genet $\mathbf{8}$, e1002599 (2012).

185. Schadt, E.E. et al. Mapping the genetic architecture of gene expression in human liver. PLoS Biol 6, e107 (2008).

186. Clayton, D.G. et al. Population structure, differential bias and genomic control in a large-scale, case-control association study. Nat Genet 37, 1243-6 (2005).

187. Heap, G.A. et al. Genome-wide analysis of allelic expression imbalance in human primary cells by high-throughput transcriptome resequencing. Hum Mol Genet 19, 122-34 (2010). 
188. Serre, D. et al. Differential allelic expression in the human genome: a robust approach to identify genetic and epigenetic cis-acting mechanisms regulating gene expression. PLoS Genet 4, e1000006 (2008).

189. Barnes, C. et al. A robust statistical method for case-control association testing with copy number variation. Nat Genet 40, 1245-52 (2008).

190. Monks, S.A. et al. Genetic inheritance of gene expression in human cell lines. Am J Hum Genet 75, 1094-105 (2004).

191. Cheung, V.G. et al. Mapping determinants of human gene expression by regional and genome-wide association. Nature 437, 1365-9 (2005).

192. Pickrell, J.K. et al. Understanding mechanisms underlying human gene expression variation with RNA sequencing. Nature 464, 768-72 (2010).

193. Montgomery, S.B. et al. Transcriptome genetics using second generation sequencing in a Caucasian population. Nature 464, 773-7 (2010).

194. Goring, H.H. et al. Discovery of expression QTLs using large-scale transcriptional profiling in human lymphocytes. Nat Genet 39, 1208-16 (2007).

195. Dixon, A.L. et al. A genome-wide association study of global gene expression. Nat Genet 39, 1202-7 (2007).

196. Gimelbrant, A., Hutchinson, J.N., Thompson, B.R. \& Chess, A. Widespread monoallelic expression on human autosomes. Science 318, 1136-40 (2007).

197. Breitling, R. et al. Genetical genomics: spotlight on QTL hotspots. PLoS Genet 4, e1000232 (2008).

198. Petretto, E. et al. Heritability and tissue specificity of expression quantitative trait loci. PLoS Genet 2, e172 (2006).

199. Luedi, P.P. et al. Computational and experimental identification of novel human imprinted genes. Genome Res 17, 1723-30 (2007). 
200. Degner, J.F. et al. DNase I sensitivity QTLs are a major determinant of human expression variation. Nature 482, 390-4 (2012).

201. Thurman, R.E. et al. The accessible chromatin landscape of the human genome. Nature 489, 75-82 (2012).

202. McDaniell, R. et al. Heritable individual-specific and allele-specific chromatin signatures in humans. Science 328, 235-9 (2010).

203. Bell, J.T. et al. DNA methylation patterns associate with genetic and gene expression variation in HapMap cell lines. Genome Biol 12, R10 (2011).

204. Pai, A.A. et al. The contribution of RNA decay quantitative trait loci to interindividual variation in steady-state gene expression levels. PLoS Genet $\mathbf{8}$, e1003000 (2012).

205. Yoon, O.K., Hsu, T.Y., Im, J.H. \& Brem, R.B. Genetics and regulatory impact of alternative polyadenylation in human B-lymphoblastoid cells. PLoS Genet $\mathbf{8}$, e1002882 (2012).

206. Rantalainen, M. et al. MicroRNA expression in abdominal and gluteal adipose tissue is associated with mRNA expression levels and partly genetically driven. PLoS ONE 6, e27338 (2011).

207. Abecasis, G.R. et al. A map of human genome variation from population-scale sequencing. Nature 467, 1061-73 (2010).

208. Rabani, M. et al. Metabolic labeling of RNA uncovers principles of RNA production and degradation dynamics in mammalian cells. Nat Biotechnol 29, 436-42 (2011).

209. Cleary, M.D., Meiering, C.D., Jan, E., Guymon, R. \& Boothroyd, J.C. Biosynthetic labeling of RNA with uracil phosphoribosyltransferase allows cellspecific microarray analysis of mRNA synthesis and decay. Nat Biotechnol 23, 232-7 (2005).

210. Blower, M.D., Feric, E., Weis, K. \& Heald, R. Genome-wide analysis demonstrates conserved localization of messenger RNAs to mitotic microtubules. J Cell Biol 179, 1365-73 (2007). 
211. Harrow, J. et al. GENCODE: the reference human genome annotation for The ENCODE Project. Genome Res 22, 1760-74 (2012).

212. Brown, C.J. et al. The human XIST gene: analysis of a $17 \mathrm{~kb}$ inactive $\mathrm{X}$-specific RNA that contains conserved repeats and is highly localized within the nucleus. Cell 71, 527-42 (1992).

213. Harris, M.E. et al. Regulation of histone mRNA in the unperturbed cell cycle: evidence suggesting control at two posttranscriptional steps. Mol Cell Biol 11, 2416-24 (1991).

214. Cowles, C.R., Hirschhorn, J.N., Altshuler, D. \& Lander, E.S. Detection of regulatory variation in mouse genes. Nat Genet 32, 432-7 (2002).

215. Patwardhan, R.P. et al. Massively parallel functional dissection of mammalian enhancers in vivo. Nat Biotechnol 30, 265-70 (2012).

216. Lefebvre, L. et al. Abnormal maternal behaviour and growth retardation associated with loss of the imprinted gene Mest. Nat Genet 20, 163-9 (1998).

217. Jiang, M. et al. The role of ZFX in non-small cell lung cancer development. Oncol Res 20, 171-8 (2012).

218. Zeggini, E. et al. Replication of genome-wide association signals in UK samples reveals risk loci for type 2 diabetes. Science 316, 1336-41 (2007).

219. Scott, L.J. et al. A genome-wide association study of type 2 diabetes in Finns detects multiple susceptibility variants. Science 316, 1341-5 (2007).

220. Diabetes Genetics Initiative of Broad Institute of, H. et al. Genome-wide association analysis identifies loci for type 2 diabetes and triglyceride levels. Science 316, 1331-6 (2007).

221. Heid, I.M. et al. Genetic architecture of the APM1 gene and its influence on adiponectin plasma levels and parameters of the metabolic syndrome in 1,727 healthy Caucasians. Diabetes 55, 375-84 (2006). 
222. Melzer, D. et al. A genome-wide association study identifies protein quantitative trait loci (pQTLs). PLoS Genet 4, e1000072 (2008).

223. Church, D.M. et al. Modernizing reference genome assemblies. PLoS Biol 9, e1001091 (2011).

224. Li, H. \& Durbin, R. Fast and accurate short read alignment with Burrows-Wheeler transform. Bioinformatics 25, 1754-60 (2009).

225. Hach, F. et al. mrsFAST: a cache-oblivious algorithm for short-read mapping. Nat Methods 7, 576-7 (2010).

226. Smit, A., Hubley, R \& Green, P. RepeatMasker Open-3.0. (1996-2010).

227. Benson, G. Tandem repeats finder: a program to analyze DNA sequences. Nucleic Acids Res 27, 573-80 (1999).

228. Alkan, C. et al. Personalized copy number and segmental duplication maps using next-generation sequencing. Nat Genet 41, 1061-7 (2009).

229. Browning, S.R. \& Browning, B.L. Rapid and accurate haplotype phasing and missing-data inference for whole-genome association studies by use of localized haplotype clustering. Am J Hum Genet 81, 1084-97 (2007).

230. Purcell, S. et al. PLINK: a tool set for whole-genome association and populationbased linkage analyses. Am J Hum Genet 81, 559-75 (2007).

231. Purcell, S., Cherny, S.S. \& Sham, P.C. Genetic Power Calculator: design of linkage and association genetic mapping studies of complex traits. Bioinformatics 19, 149-50 (2003).

232. Team, R.D.C. R: A Language and Environment for Statistical Computing. (2012).

233. Kenzelmann, M. et al. Microarray analysis of newly synthesized RNA in cells and animals. Proc Natl Acad Sci U S A 104, 6164-9 (2007).

234. Dolken, L. et al. High-resolution gene expression profiling for simultaneous kinetic parameter analysis of RNA synthesis and decay. RNA 14, 1959-72 (2008). 
235. Cleary, M.D. Cell type-specific analysis of mRNA synthesis and decay in vivo with uracil phosphoribosyltransferase and 4-thiouracil. Methods Enzymol 448, 379-406 (2008).

236. Stephens, S.B., Dodd, R.D., Lerner, R.S., Pyhtila, B.M. \& Nicchitta, C.V. Analysis of mRNA partitioning between the cytosol and endoplasmic reticulum compartments of mammalian cells. Methods Mol Biol 419, 197-214 (2008).

237. Trapnell, C., Pachter, L. \& Salzberg, S.L. TopHat: discovering splice junctions with RNA-Seq. Bioinformatics 25, 1105-11 (2009).

238. Koren, A. \& McCarroll, S.A. Random replication of the inactive X chromosome. Genome Res 24, 64-9 (2014). 